\title{
ENOLS OF SUBSTITUTED CYANOMALONAMIDES
}

Ahmad Basheer, Hiroshi Yamataka, Salai Cheettu Ammal and Zvi Rappoport

Supporting information

Table of contents

Table of contents

Table S1

Table S2

Table S3

Table S4

Table S5

Table S6

${ }^{1} \mathrm{H}$ NMR of a mixture of $\mathrm{R}^{3} \mathrm{NHCOCH}(\mathrm{CN}) \mathrm{CONR}^{1} \mathrm{R}^{2}$ (4) and

their enols (5/6)in several solvents ${ }^{\mathrm{a}}$

${ }^{1} \mathrm{H}$ NMR of a mixture of $\mathrm{PhNHCOCH}(\mathrm{CN}) \mathrm{CONMe}_{2}$ and its enol in $\mathrm{CDCl}_{3}$

${ }^{1} \mathrm{H}$ NMR of a mixture of $p-\mathrm{AnNHCOCH}(\mathrm{CN}) \mathrm{CONMe}_{2}$ and its enol in $\mathrm{CD}_{3} \mathrm{CN}$ 
${ }^{1} \mathrm{H}$ NMR of a mixture of $\mathrm{C}_{6} \mathrm{~F}_{5} \mathrm{NHCOCH}(\mathrm{CN}) \mathrm{CONMe}_{2}$ and its enol in $\mathrm{CD}_{3} \mathrm{CN}$

${ }^{1} \mathrm{H}$ NMR of a mixture of $i-\mathrm{PrNHCOCH}(\mathrm{CN}) \mathrm{CONMe}_{2}$ and its enol in $\mathrm{CDCl}_{3}$

${ }^{1} \mathrm{H}$ NMR of a mixture of $t-\mathrm{BuNHCOCH}(\mathrm{CN}) \mathrm{CONMe}_{2}$ and its enol in $\mathrm{CDCl}_{3}$

${ }^{1} \mathrm{H}$ NMR of a mixture of $\mathrm{Ph}_{3} \mathrm{CNHCOCH}(\mathrm{CN}) \mathrm{CONMe}_{2}$ and its enol in $\mathrm{CDCl}_{3}$

${ }^{1} \mathrm{H}$ NMR of a mixture of $\mathrm{PhNHCOCH}(\mathrm{CN}) \mathrm{CONHMe}$ and its enol in $\mathrm{C}_{6} \mathrm{D}_{6}$

${ }^{1} \mathrm{H}$ NMR of a mixture of $p-\mathrm{AnNHCOCH}(\mathrm{CN}) \mathrm{CONHMe}$ and its enol in $\mathrm{CDCl}_{3}$

${ }^{1} \mathrm{H}$ NMR of a mixture of $\mathrm{C}_{6} \mathrm{~F}_{5} \mathrm{NHCOCH}(\mathrm{CN}) \mathrm{CONHMe}$ and its enol in $\mathrm{CD}_{3} \mathrm{CN}$

${ }^{1} \mathrm{H}$ NMR of a mixture of $i-\mathrm{PrNHCOCH}(\mathrm{CN}) \mathrm{CONHMe}$ and its enol in $\mathrm{CDCl}_{3}$

${ }^{1} \mathrm{H}$ NMR of a mixture of $t-\mathrm{BuNHCOCH}(\mathrm{CN}) \mathrm{CONHMe}$ and its enol in $\mathrm{CDCl}_{3}$

${ }^{1} \mathrm{H}$ NMR of a mixture of $\mathrm{PhNHCOCH}(\mathrm{CN}) \mathrm{CONH}_{2}$ and its enol in $\mathrm{CD}_{3} \mathrm{CN}$

${ }^{1} \mathrm{H}$ NMR of a mixture of $p-A n N H C O C H(C N) C_{2 N H}$ and its enol in THF-d

${ }^{1} \mathrm{H}$ NMR of a mixture of $\mathrm{C}_{6} \mathrm{~F}_{5} \mathrm{NHCOCH}(\mathrm{CN}) \mathrm{CONH}_{2}$ and its enol in THF-d 8

${ }^{1} \mathrm{H}$ NMR of a mixture of $i-\mathrm{PrNHCOCH}(\mathrm{CN}) \mathrm{CONH}_{2}$ and its enol in $\mathrm{CDCl}_{3}$

${ }^{1} \mathrm{H}$ NMR of a mixture of $t$-BuNHCOCH(CN)CONH$H_{2}$ and its enol in $\mathrm{C}_{6} \mathrm{D}_{6}$

${ }^{1} \mathrm{H}$ NMR of a mixture of $\mathrm{C}_{6} \mathrm{~F}_{5} \mathrm{NHCOCH}(\mathrm{CN}) \mathrm{CONHPh}$ and its enol in THF-d

${ }^{1} \mathrm{H}$ NMR of a mixture of PhNHCOCH$(\mathrm{CN}) \mathrm{CONHPh}$ and its enol in THF-d

${ }^{1} \mathrm{H}$ NMR of a mixture of $i-\mathrm{PrNHCOCH}(\mathrm{CN}) \mathrm{CONHMe}$ and its enol in THF-d 8 
cif file of $6 \mathbf{a}$ at $295 \mathrm{~K}$

cif file of $6 \mathrm{a}$ at $123 \mathrm{~K}$

cif file of $\mathbf{4 b}$ at $295 \mathrm{~K}$

cif file of $6 c$ at $295 \mathrm{~K}$

cif file of $\mathbf{4 d}$ at $298 \mathrm{~K}$

cif file of $\mathbf{4 g}$ at $100 \mathrm{~K}$

cif file of $5 \mathrm{~h}$ at $295 \mathrm{~K}$

cif file of $5 \mathrm{~h}$ at $123 \mathrm{~K}$

cif file of $6 \mathbf{i}$ at $173 \mathrm{~K}$

cif file of $\mathbf{5 j} / \mathbf{6 j}$ at $295 \mathrm{~K}$

cif file of $\mathbf{4 k}$ at $295 \mathrm{~K}$

cif file of $5 n$ at $123 \mathrm{~K}$

cif file of 50 at $100 \mathrm{~K}$

cif file of $6 q$ at $295 \mathrm{~K}$

cif file of $5 \mathbf{s} / 6 \mathbf{s}$ at $123 \mathrm{~K}$

$\overline{\text { a }}$ The spectra are mostly of a mixtures of both species, the structure of only one of them (either amide or enol) is drown on the spectrum. 
Table S1. Enol/Amide Ratios Based on Integration of Various Signals for the $N C C H\left(C O N R^{1} R^{2}\right)\left(C O N R^{3} R^{4}\right) / R^{3} R^{4} N C O C(C N)=C(O H) N R R^{1}$ system in Several Solvents.

\begin{tabular}{|c|c|c|c|c|c|c|c|}
\hline $\mathrm{R}^{1}$ & $\mathrm{R}^{2}$ & $\mathrm{R}^{3}$ & $\mathrm{R}^{4}$ & Solvent & Signal $^{\mathrm{a}}$ & $\%$ Enol & $\%$ Amide \\
\hline \multirow[t]{10}{*}{$\mathrm{H}$} & $\mathrm{Ph}$ & $\mathrm{H}$ & $\mathrm{Ph}$ & THF- $\mathrm{d}_{8}$ & $\mathrm{OH}, \mathrm{CH}$ & 67 & 33 \\
\hline & & & & & $\mathrm{Ph}-\mathrm{H}$ & 70 & 30 \\
\hline & & & & & $\mathrm{NH}$ & 71 & 29 \\
\hline & & & & & Average & 69 & 31 \\
\hline & & & & $\mathrm{CD}_{3} \mathrm{CN}$ & $\mathrm{OH}, \mathrm{CH}$ & 33 & 67 \\
\hline & & & & & $\mathrm{Ph}-\mathrm{H}$ & 35 & 65 \\
\hline & & & & & $\mathrm{N}-\mathrm{H}$ & 33 & 67 \\
\hline & & & & & Average & 34 & 66 \\
\hline & & & & DMF-d ${ }_{7}$ & All signals & 0 & 100 \\
\hline & & & & $\mathrm{DMSO}^{-\mathrm{d}_{6}}$ & All signals & 0 & 100 \\
\hline \multirow[t]{14}{*}{$\mathrm{H}$} & $i-\operatorname{Pr}$ & $\mathrm{H}$ & $i$-Pr & $\mathrm{CDCl}_{3}$ & $\mathrm{OH}, \mathrm{CH}$ & 88 & 12 \\
\hline & & & & & $\mathrm{NH}$ & 90 & 10 \\
\hline & & & & & Average & 89 & 11 \\
\hline & & & & $\mathrm{C}_{6} \mathrm{D}_{6}$ & $\operatorname{Me}(i-\operatorname{Pr})$ & 94 & 6 \\
\hline & & & & & $\mathrm{NH}$ & 94 & 6 \\
\hline & & & & & Average & 94 & 6 \\
\hline & & & & THF- $\mathrm{d}_{8}$ & $\operatorname{Me}(i-\operatorname{Pr})$ & 81 & 19 \\
\hline & & & & & $\mathrm{CH}(i-\mathrm{Pr})$ & 86 & 14 \\
\hline & & & & & $\mathrm{OH}, \mathrm{CH}$ & 85 & 15 \\
\hline & & & & & $\mathrm{NH}$ & 87 & 13 \\
\hline & & & & & Average & 85 & 15 \\
\hline & & & & $\mathrm{CD}_{3} \mathrm{CN}$ & $\operatorname{Me}(i-\operatorname{Pr})$ & 40 & 60 \\
\hline & & & & & $\mathrm{CH}(i-\mathrm{Pr})$ & 44 & 56 \\
\hline & & & & & $\mathrm{CH}, \mathrm{OH}$ & 44 & 56 \\
\hline
\end{tabular}




\begin{tabular}{|c|c|c|c|c|c|c|c|}
\hline & & & & & $\mathrm{NH}$ & 46 & 54 \\
\hline & & & & \multirow{6}{*}{ DMF-d 7} & Average & 44 & 56 \\
\hline & & & & & $\operatorname{Me}(i-\operatorname{Pr})$ & 37 & 63 \\
\hline & & & & & $\mathrm{CH}(i-\mathrm{Pr})$ & 38 & 62 \\
\hline & & & & & $\mathrm{CH}, \mathrm{OH}$ & 37 & 63 \\
\hline & & & & & $\mathrm{NH}$ & 39 & 61 \\
\hline & & & & & Average & 38 & 62 \\
\hline & & & & \multirow{5}{*}{$\mathrm{DMSO}^{-\mathrm{d}_{6}}$} & $\operatorname{Me}(i-\operatorname{Pr})$ & 20 & 80 \\
\hline & & & & & $\mathrm{CH}(i-\mathrm{Pr})$ & 20 & 80 \\
\hline & & & & & $\mathrm{CH}, \mathrm{OH}$ & 18 & 82 \\
\hline & & & & & $\mathrm{NH}$ & 17 & 83 \\
\hline & & & & & Average & 19 & 81 \\
\hline \multirow[t]{12}{*}{$\mathrm{H}$} & \multirow[t]{12}{*}{$\mathrm{CHPh}_{2}$} & \multirow[t]{12}{*}{$\mathrm{H}$} & \multirow[t]{12}{*}{$\mathrm{CHPh}_{2}$} & $\mathrm{CDCl}_{3}$ & All signals & 100 & 0 \\
\hline & & & & $\mathrm{C}_{6} \mathrm{D}_{6}$ & All signals & 100 & 0 \\
\hline & & & & THF-d ${ }_{8}$ & $\mathrm{OH}, \mathrm{CH}$ & 55 & 45 \\
\hline & & & & & $\mathrm{CHPh}_{2}$ & 63 & 37 \\
\hline & & & & & $\mathrm{NH}$ & 68 & 32 \\
\hline & & & & & Average & 62 & 38 \\
\hline & & & & $\mathrm{CD}_{3} \mathrm{CN}$ & $\mathrm{OH}, \mathrm{CH}$ & 13 & 87 \\
\hline & & & & & $\mathrm{CHPh}$ & 13 & 87 \\
\hline & & & & & $\mathrm{NH}$ & 14 & 86 \\
\hline & & & & & Average & 13 & 87 \\
\hline & & & & DMF-d ${ }_{7}$ & All signals & 0 & 100 \\
\hline & & & & $\mathrm{DMSO}^{-\mathrm{d}_{6}}$ & All signals & 0 & 100 \\
\hline \multirow[t]{9}{*}{$\mathrm{H}$} & \multirow[t]{9}{*}{$\mathrm{C}_{6} \mathrm{H}_{5}$} & \multirow[t]{9}{*}{$\mathrm{H}$} & \multirow[t]{9}{*}{$\mathrm{C}_{6} \mathrm{~F}_{5}$} & $\mathrm{CDCl}_{3}$ & All signals & 100 & 0 \\
\hline & & & & $\mathrm{C}_{6} \mathrm{D}_{6}$ & All signals & 100 & 0 \\
\hline & & & & THF- $\mathrm{d}_{8}$ & $\mathrm{OH}, \mathrm{CH}$ & 87 & 13 \\
\hline & & & & & $\mathrm{Ph}-\mathrm{H}$ & 84 & 16 \\
\hline & & & & & $\mathrm{Ph}-\mathrm{H}$ & 88 & 12 \\
\hline & & & & & $\mathrm{NH}(1)$ & 89 & 11 \\
\hline & & & & & NH (2) & 88 & 12 \\
\hline & & & & & Average & 87 & 13 \\
\hline & & & & $\mathrm{CD}_{3} \mathrm{CN}$ & $\mathrm{OH}, \mathrm{CH}$ & 54 & 46 \\
\hline
\end{tabular}




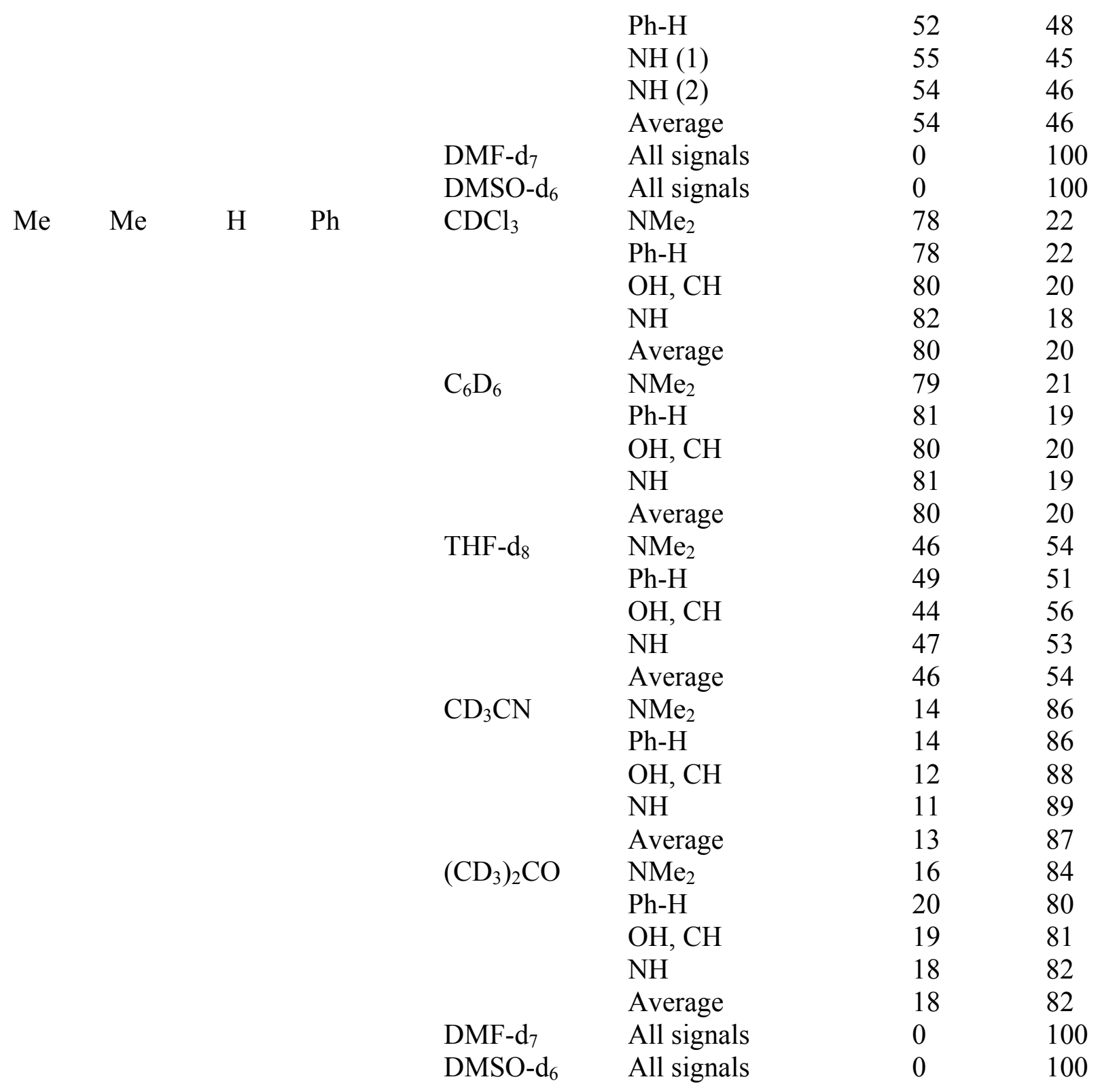




\begin{tabular}{|c|c|c|c|c|c|c|c|}
\hline \multirow[t]{27}{*}{$\mathrm{Me}$} & \multirow[t]{27}{*}{$\mathrm{Me}$} & \multirow[t]{27}{*}{$\mathrm{H}$} & \multirow[t]{27}{*}{$p-\mathrm{MeOC}_{6} \mathrm{H}_{4}$} & \multirow[t]{5}{*}{$\mathrm{CDCl}_{3}$} & $\mathrm{NMe}_{2}$ & 80 & 20 \\
\hline & & & & & Ar-H & 78 & 22 \\
\hline & & & & & $\mathrm{OH}, \mathrm{CH}$ & 80 & 20 \\
\hline & & & & & $\mathrm{NH}$ & 83 & 23 \\
\hline & & & & & Average & 80 & 20 \\
\hline & & & & \multirow[t]{6}{*}{$\mathrm{C}_{6} \mathrm{D}_{6}$} & $\mathrm{NMe}_{2}$ & 77 & 23 \\
\hline & & & & & $\mathrm{OMe}$ & 75 & 25 \\
\hline & & & & & Ar-H & 79 & 21 \\
\hline & & & & & $\mathrm{OH}, \mathrm{CH}$ & 78 & 22 \\
\hline & & & & & $\mathrm{NH}$ & 80 & 20 \\
\hline & & & & & Average & 78 & 22 \\
\hline & & & & \multirow[t]{6}{*}{ THF-d 8} & $\mathrm{NMe}_{2}$ & 41 & 59 \\
\hline & & & & & $\mathrm{OMe}$ & 42 & 58 \\
\hline & & & & & Ar-H & 47 & 53 \\
\hline & & & & & $\mathrm{OH}, \mathrm{CH}$ & 41 & 59 \\
\hline & & & & & $\mathrm{NH}$ & 45 & 55 \\
\hline & & & & & Average & 44 & 56 \\
\hline & & & & \multirow[t]{5}{*}{$\mathrm{CD}_{3} \mathrm{CN}$} & $\mathrm{NMe}_{2}$ & 13 & 87 \\
\hline & & & & & Ar-H & 14 & 86 \\
\hline & & & & & $\mathrm{OH}, \mathrm{CH}$ & 12 & 88 \\
\hline & & & & & $\mathrm{NH}$ & 12 & 88 \\
\hline & & & & & Average & 13 & 87 \\
\hline & & & & \multirow[t]{4}{*}{ DMF-d 7} & $\mathrm{NMe}_{2}$ & 6 & 94 \\
\hline & & & & & $\mathrm{OH}, \mathrm{CH}$ & 4 & 96 \\
\hline & & & & & $\mathrm{NH}$ & 5 & 95 \\
\hline & & & & & Average & 5 & 95 \\
\hline & & & & DMSO- $\mathrm{d}_{6}$ & All signals & 0 & 100 \\
\hline \multirow[t]{6}{*}{$\mathrm{Me}$} & \multirow[t]{6}{*}{$\mathrm{Me}$} & \multirow[t]{6}{*}{$\mathrm{H}$} & \multirow[t]{6}{*}{$\mathrm{C}_{6} \mathrm{~F}_{5}$} & \multirow[t]{3}{*}{$\mathrm{CDCl}_{3}$} & $\mathrm{OH}, \mathrm{CH}$ & 93 & 7 \\
\hline & & & & & $\mathrm{NH}$ & 94 & 6 \\
\hline & & & & & Average & 93 & 7 \\
\hline & & & & \multirow[t]{3}{*}{$\mathrm{C}_{6} \mathrm{D}_{6}$} & $\mathrm{OH}, \mathrm{CH}$ & 94 & 6 \\
\hline & & & & & $\mathrm{NH}$ & 95 & 5 \\
\hline & & & & & Average & 95 & 5 \\
\hline
\end{tabular}




\begin{tabular}{|c|c|c|c|c|c|c|c|}
\hline & & & & THF- $\mathrm{d}_{8}$ & $\mathrm{NMe}_{2}$ & 74 & 26 \\
\hline & & & & & $\mathrm{OH}, \mathrm{CH}$ & 78 & 22 \\
\hline & & & & & $\mathrm{NH}$ & 80 & 20 \\
\hline & & & & & Average & 77 & 23 \\
\hline & & & & $\mathrm{CD}_{3} \mathrm{CN}$ & $\mathrm{NMe}_{2}$ & 42 & 58 \\
\hline & & & & & $\mathrm{OH}, \mathrm{CH}$ & 37 & 63 \\
\hline & & & & & $\mathrm{NH}$ & 38 & 62 \\
\hline & & & & & Average & 39 & 61 \\
\hline & & & & DMF-d $_{7}$ & $\mathrm{NMe}_{2}$ & 15 & 85 \\
\hline & & & & & $\mathrm{OH}, \mathrm{CH}$ & 14 & 86 \\
\hline & & & & & $\mathrm{NH}$ & 17 & 83 \\
\hline & & & & & Average & 15 & 85 \\
\hline & & & & $\mathrm{DMSO}_{-} \mathrm{d}_{6}$ & $\mathrm{NMe}_{2}$ & 9 & 91 \\
\hline & & & & & $\mathrm{OH}, \mathrm{CH}$ & 5 & 95 \\
\hline & & & & & $\mathrm{NH}$ & 6 & 94 \\
\hline & & & & & Average & 7 & 93 \\
\hline $\mathrm{Me}$ & $\mathrm{Me}$ & $\mathrm{H}$ & $i$-Pr & $\mathrm{CDCl}_{3}$ & $\mathrm{NMe}_{2}$ & 78 & 22 \\
\hline & & & & & $\operatorname{Me}(i-\operatorname{Pr})$ & 79 & 21 \\
\hline & & & & & $\mathrm{OH}, \mathrm{CH}$ & 80 & 20 \\
\hline & & & & & $\mathrm{NH}$ & 81 & 19 \\
\hline & & & & & Average & 80 & 20 \\
\hline & & & & $\mathrm{C}_{6} \mathrm{D}_{6}$ & $\mathrm{NMe}_{2}$ & 82 & 18 \\
\hline & & & & & $\mathrm{OH}, \mathrm{CH}$ & 84 & 16 \\
\hline & & & & & $\mathrm{NH}$ & 83 & 17 \\
\hline & & & & & Average & 83 & 17 \\
\hline & & & & THF- $\mathrm{d}_{8}$ & $\mathrm{NMe}_{2}$ & 61 & 39 \\
\hline & & & & & $\mathrm{Me}(i-\mathrm{Pr})$ & 65 & 35 \\
\hline & & & & & $\mathrm{CH}(i-\mathrm{Pr})$ & 64 & 36 \\
\hline & & & & & $\mathrm{NH}$ & 62 & 38 \\
\hline & & & & & Average & 63 & 37 \\
\hline & & & & $\left(\mathrm{CD}_{3}\right)_{2} \mathrm{CO}$ & $\mathrm{NMe}_{2}$ & 27 & 73 \\
\hline & & & & & $\mathrm{CH}(i-\mathrm{Pr})$ & 25 & 75 \\
\hline & & & & & $\mathrm{OH}, \mathrm{CH}$ & 30 & 70 \\
\hline
\end{tabular}




\begin{tabular}{|c|c|c|c|c|c|c|c|}
\hline & & & & & NH & 29 & 71 \\
\hline & & & & & Average & 28 & 72 \\
\hline & & & & $\mathrm{CD}_{3} \mathrm{CN}$ & $\mathrm{NMe}_{2}$ & 14 & 86 \\
\hline & & & & & $\mathrm{Me}(i-\mathrm{Pr})$ & 16 & 84 \\
\hline & & & & & $\mathrm{CH}(i$-Pr $)$ & 13 & 87 \\
\hline & & & & & $\mathrm{OH}, \mathrm{CH}$ & 12 & 88 \\
\hline & & & & & $\mathrm{NH}$ & 12 & 88 \\
\hline & & & & & Average & 13 & 87 \\
\hline & & & & DMF- $\mathrm{d}_{7}$ & $\mathrm{NMe}_{2}$ & 8 & 92 \\
\hline & & & & & $\operatorname{Me}(i-\operatorname{Pr})$ & 8 & 92 \\
\hline & & & & & $\mathrm{CH}(i-\mathrm{Pr})$ & 6 & 94 \\
\hline & & & & & $\mathrm{NH}$ & 4 & 96 \\
\hline & & & & & Average & 7 & 93 \\
\hline & & & & DMSO- $\mathrm{d}_{6}$ & $\mathrm{NMe}_{2}$ & 3 & 97 \\
\hline & & & & & $\operatorname{Me}(i-\operatorname{Pr})$ & 4 & 96 \\
\hline & & & & & $\mathrm{CH}(i$-Pr) & 4 & 96 \\
\hline & & & & & $\mathrm{OH}, \mathrm{CH}$ & 2 & 98 \\
\hline & & & & & $\mathrm{NH}$ & 8 & 92 \\
\hline & & & & & Average & 4 & 96 \\
\hline $\mathrm{Me}$ & $\mathrm{Me}$ & $\mathrm{H}$ & $t-\mathrm{Bu}$ & $\mathrm{CDCl}_{3}$ & $t-\mathrm{Bu}$ & 77 & 23 \\
\hline & & & & & $\mathrm{NMe}_{2}$ & 72 & 28 \\
\hline & & & & & $\mathrm{OH}, \mathrm{CH}$ & 72 & 28 \\
\hline & & & & & $\mathrm{NH}$ & 72 & 28 \\
\hline & & & & & Average & 73 & 27 \\
\hline & & & & $\mathrm{C}_{6} \mathrm{D}_{6}$ & $t-\mathrm{Bu}$ & 80 & 20 \\
\hline & & & & & $\mathrm{NMe}_{2}$ & 78 & 22 \\
\hline & & & & & $\mathrm{OH}, \mathrm{CH}$ & 80 & 20 \\
\hline & & & & & $\mathrm{NH}$ & 80 & 20 \\
\hline & & & & & Average & 80 & 20 \\
\hline & & & & THF- $\mathrm{d}_{8}$ & $t-\mathrm{Bu}$ & 47 & 53 \\
\hline & & & & & $\mathrm{NMe}_{2}$ & 47 & 53 \\
\hline & & & & & $\mathrm{OH}, \mathrm{CH}$ & 49 & 51 \\
\hline & & & & & $\mathrm{NH}$ & 46 & 54 \\
\hline
\end{tabular}




\begin{tabular}{|c|c|c|c|c|c|c|}
\hline & & & & Average & 47 & 53 \\
\hline & & & $\mathrm{CD}_{3} \mathrm{CN}$ & $t-\mathrm{Bu}$ & 11 & 89 \\
\hline & & & & $\mathrm{NMe}_{2}$ & 8 & 92 \\
\hline & & & & $\mathrm{OH}, \mathrm{CH}$ & 8 & 92 \\
\hline & & & & $\mathrm{NH}$ & 3 & 97 \\
\hline & & & & Average & 8 & 92 \\
\hline & & & $\mathrm{DMF}^{-\mathrm{d}_{7}}$ & $t-\mathrm{Bu}$ & 3 & 97 \\
\hline & & & & $\mathrm{NMe}_{2}$ & 2 & 98 \\
\hline & & & & $\mathrm{OH}, \mathrm{CH}$ & 1 & 99 \\
\hline & & & & NH & 1 & 99 \\
\hline & & & & Average & 2 & 98 \\
\hline & & & DMSO-d $\mathrm{d}_{6}$ & All signals & 0 & 100 \\
\hline $\mathrm{Me}$ & $\mathrm{H}$ & $\mathrm{Ph}$ & $\mathrm{CDCl}_{3}$ & $\mathrm{NMe}$ & 90 & 10 \\
\hline & & & & $\mathrm{OH}, \mathrm{CH}$ & 90 & 10 \\
\hline & & & & $\mathrm{NH}$ & 90 & 10 \\
\hline & & & & Average & 90 & 10 \\
\hline & & & $\mathrm{C}_{6} \mathrm{D}_{6}$ & $\mathrm{NMe}$ & 91 & 9 \\
\hline & & & & $\mathrm{OH}, \mathrm{CH}$ & 92 & 8 \\
\hline & & & & $\mathrm{NH}$ & 92 & 8 \\
\hline & & & & Average & 92 & 8 \\
\hline & & & THF-d 8 & $\mathrm{NMe}$ & 81 & 19 \\
\hline & & & & $\mathrm{OH}, \mathrm{CH}$ & 85 & 15 \\
\hline & & & & $\mathrm{NH}$ & 87 & 13 \\
\hline & & & & Average & 84 & 16 \\
\hline & & & $\mathrm{CD}_{3} \mathrm{CN}$ & $\mathrm{NMe}$ & 52 & 48 \\
\hline & & & & $\mathrm{OH}, \mathrm{CH}$ & 51 & 49 \\
\hline & & & & NH (I) & 51 & 49 \\
\hline & & & & NH (II) & 54 & 46 \\
\hline & & & & $\mathrm{Ph}-\mathrm{H}$ & 52 & 48 \\
\hline & & & & Average & 52 & 48 \\
\hline & & & DMF-d $\mathrm{d}_{7}$ & $\mathrm{NMe}$ & 33 & 67 \\
\hline & & & & $\mathrm{OH}, \mathrm{CH}$ & 25 & 75 \\
\hline & & & & $\mathrm{NH}$ & 29 & 71 \\
\hline
\end{tabular}




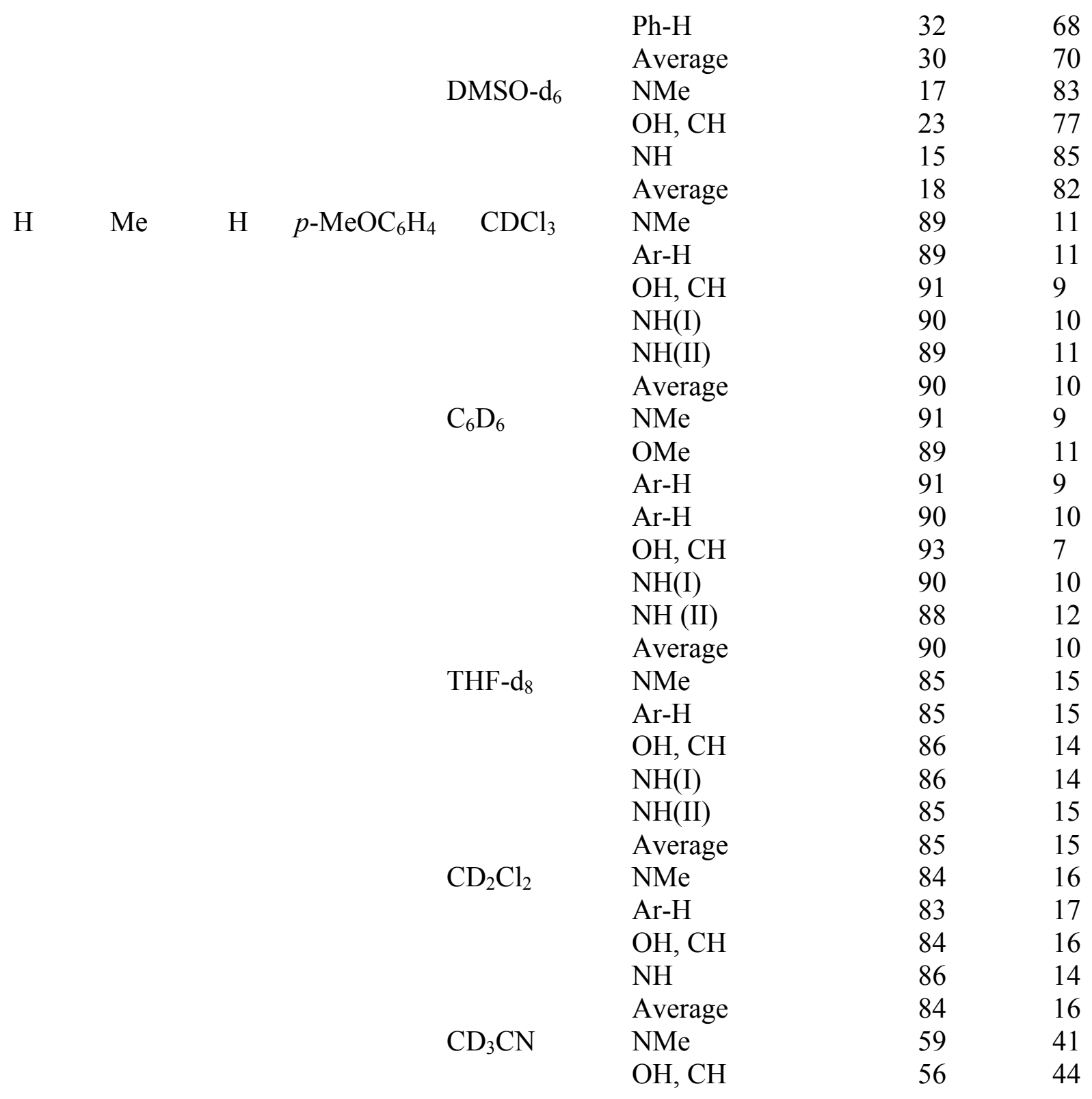




\begin{tabular}{|c|c|c|c|c|c|c|}
\hline & & & & $\mathrm{NH}$ & 55 & 45 \\
\hline & & & & Average & 57 & 43 \\
\hline & & & DMF-d $\mathrm{d}_{7}$ & $\mathrm{NMe}$ & 30 & 70 \\
\hline & & & & Ar-H & 35 & 65 \\
\hline & & & & $\mathrm{OH}, \mathrm{CH}$ & 28 & 72 \\
\hline & & & & $\mathrm{NH}$ & 30 & 70 \\
\hline & & & & Average & 31 & 69 \\
\hline & & & DMSO-d $\mathrm{d}_{6}$ & $\mathrm{NMe}$ & 18 & 82 \\
\hline & & & & Ar-H & 17 & 83 \\
\hline & & & & $\mathrm{OH}, \mathrm{CH}$ & 13 & 87 \\
\hline & & & & $\mathrm{NH}$ & 17 & 83 \\
\hline & & & & Average & 16 & 84 \\
\hline $\mathrm{Me}$ & $\mathrm{H}$ & $\mathrm{C}_{6} \mathrm{~F}_{5}$ & $\mathrm{CDCl}_{3}$ & $\mathrm{NMe}$ & 96 & 4 \\
\hline & & & & $\mathrm{OH}, \mathrm{CH}$ & 97 & 3 \\
\hline & & & & NH (I) & 96 & 4 \\
\hline & & & & NH (II) & 98 & 2 \\
\hline & & & & Average & 97 & 3 \\
\hline & & & $\mathrm{C}_{6} \mathrm{D}_{6}$ & $\mathrm{NMe}$ & 96 & 4 \\
\hline & & & & $\mathrm{OH}, \mathrm{CH}$ & 97 & 3 \\
\hline & & & & NH (I) & 97 & 3 \\
\hline & & & & NH (II) & 98 & 2 \\
\hline & & & & Average & 97 & 3 \\
\hline & & & THF-d 8 & $\mathrm{NMe}$ & 95 & 5 \\
\hline & & & & $\mathrm{OH}, \mathrm{CH}$ & 96 & 4 \\
\hline & & & & NH (I) & 96 & 4 \\
\hline & & & & NH (II) & 97 & 3 \\
\hline & & & & Average & 96 & 4 \\
\hline & & & $\mathrm{CD}_{3} \mathrm{CN}$ & $\mathrm{NMe}$ & 81 & 19 \\
\hline & & & & $\mathrm{OH}, \mathrm{CH}$ & 77 & 23 \\
\hline & & & & NH (I) & 78 & 22 \\
\hline & & & & NH (II) & 80 & 20 \\
\hline & & & & Average & 79 & \\
\hline & & & DMF-d $\mathrm{d}_{7}$ & $\mathrm{NMe}$ & 70 & \\
\hline
\end{tabular}




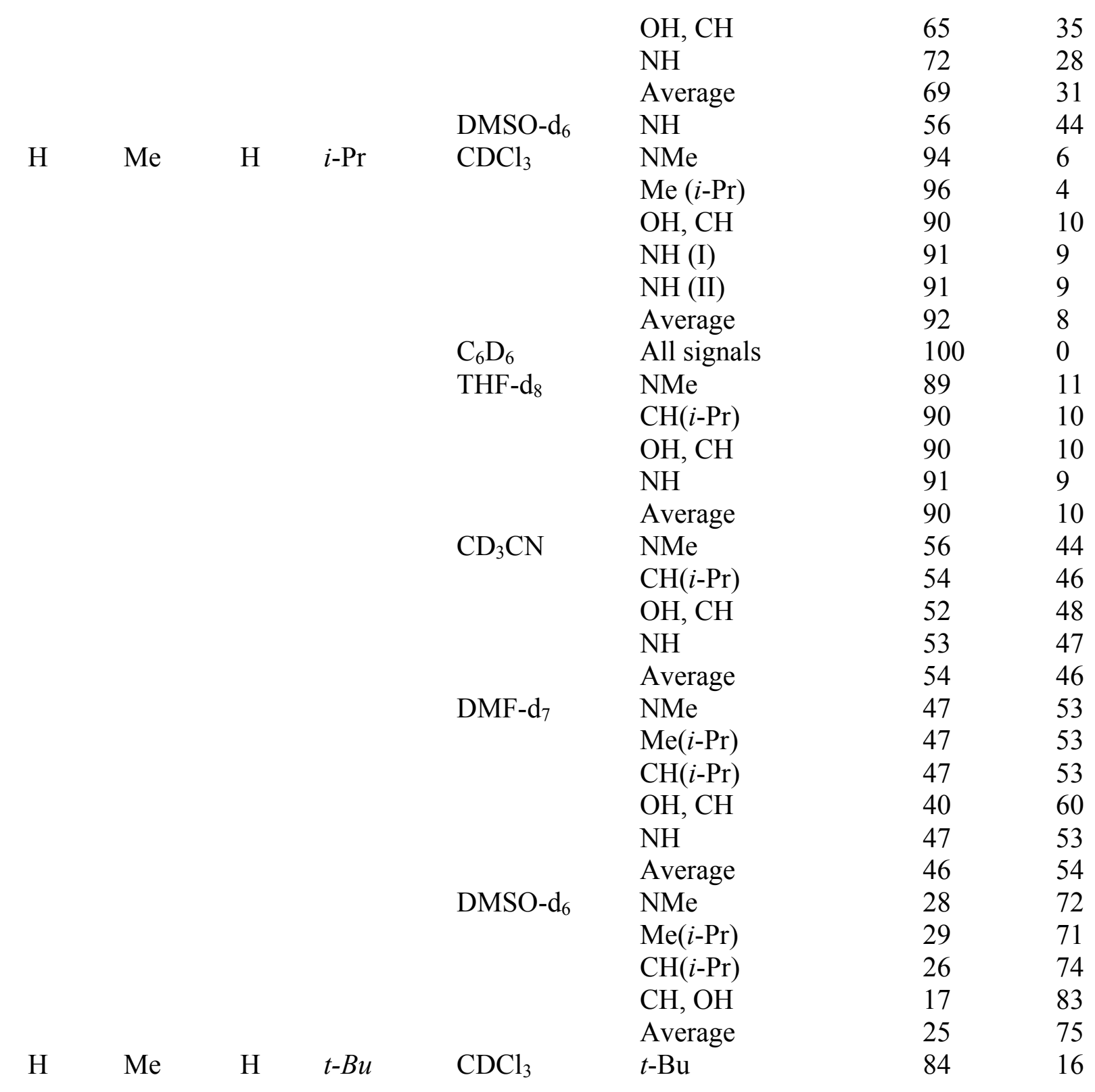




\begin{tabular}{|c|c|c|c|c|c|c|c|}
\hline & & & & & $\mathrm{OH}, \mathrm{CH}$ & 83 & 17 \\
\hline & & & & & $\mathrm{NH}$ & 85 & 15 \\
\hline & & & & & Average & 84 & 16 \\
\hline & & & & $\mathrm{C}_{6} \mathrm{D}_{6}$ & $\mathrm{NMe}$ & 96 & 4 \\
\hline & & & & & $\mathrm{t}-\mathrm{Bu}$ & 92 & 8 \\
\hline & & & & & $\mathrm{OH}, \mathrm{CH}$ & 92 & 8 \\
\hline & & & & & NH (I) & 93 & 7 \\
\hline & & & & & NH (II) & 93 & 7 \\
\hline & & & & & Average & 93 & 7 \\
\hline & & & & THF-d 8 & $\mathrm{OH}, \mathrm{CH}$ & 76 & 24 \\
\hline & & & & DMSO-d $\mathrm{d}_{6}$ & $\mathrm{NMe}$ & 27 & 73 \\
\hline & & & & & $t$-Bu & 27 & 73 \\
\hline & & & & & Average & 27 & 73 \\
\hline $\mathrm{H}$ & $\mathrm{H}$ & $\mathrm{H}$ & $\mathrm{Ph}$ & $\mathrm{CDCl}_{3}$ & All signals & 100 & 0 \\
\hline & & & & $\mathrm{C}_{6} \mathrm{D}_{6}$ & All signals & 100 & 0 \\
\hline & & & & THF- $\mathrm{d}_{8}$ & $\mathrm{Ph}-\mathrm{H}$ & 96 & 4 \\
\hline & & & & & $\mathrm{OH}, \mathrm{CH}$ & 98 & 2 \\
\hline & & & & & $\mathrm{NH}$ & 97 & 3 \\
\hline & & & & & Average & 97 & 3 \\
\hline & & & & $\mathrm{CD}_{3} \mathrm{CN}$ & $\mathrm{Ph}-\mathrm{H}$ & 81 & 19 \\
\hline & & & & & $\mathrm{OH}, \mathrm{CH}$ & 83 & 17 \\
\hline & & & & & $\mathrm{NH}$ & 84 & 16 \\
\hline & & & & & Average & 83 & 17 \\
\hline & & & & DMF-d $\mathrm{d}_{7}$ & $\mathrm{OH}, \mathrm{CH}$ & 68 & 32 \\
\hline & & & & & $\mathrm{NH}$ & 70 & 30 \\
\hline & & & & & Average & 69 & 31 \\
\hline & & & & DMSO-d 6 & $\mathrm{OH}, \mathrm{CH}$ & 43 & 57 \\
\hline $\mathrm{H}$ & $\mathrm{H}$ & $\mathrm{H}$ & $p-\mathrm{MeOC}_{6} \mathrm{H}_{4}$ & THF- $\mathrm{d}_{8}$ & $\mathrm{OMe}$ & 92 & 8 \\
\hline & & & & & Ar-H & 91 & 9 \\
\hline & & & & & Ar-H & 95 & 5 \\
\hline & & & & & $\mathrm{OH}, \mathrm{CH}$ & 97 & 3 \\
\hline & & & & & $\mathrm{NH}$ & 94 & 6 \\
\hline & & & & & Average & 94 & 6 \\
\hline
\end{tabular}




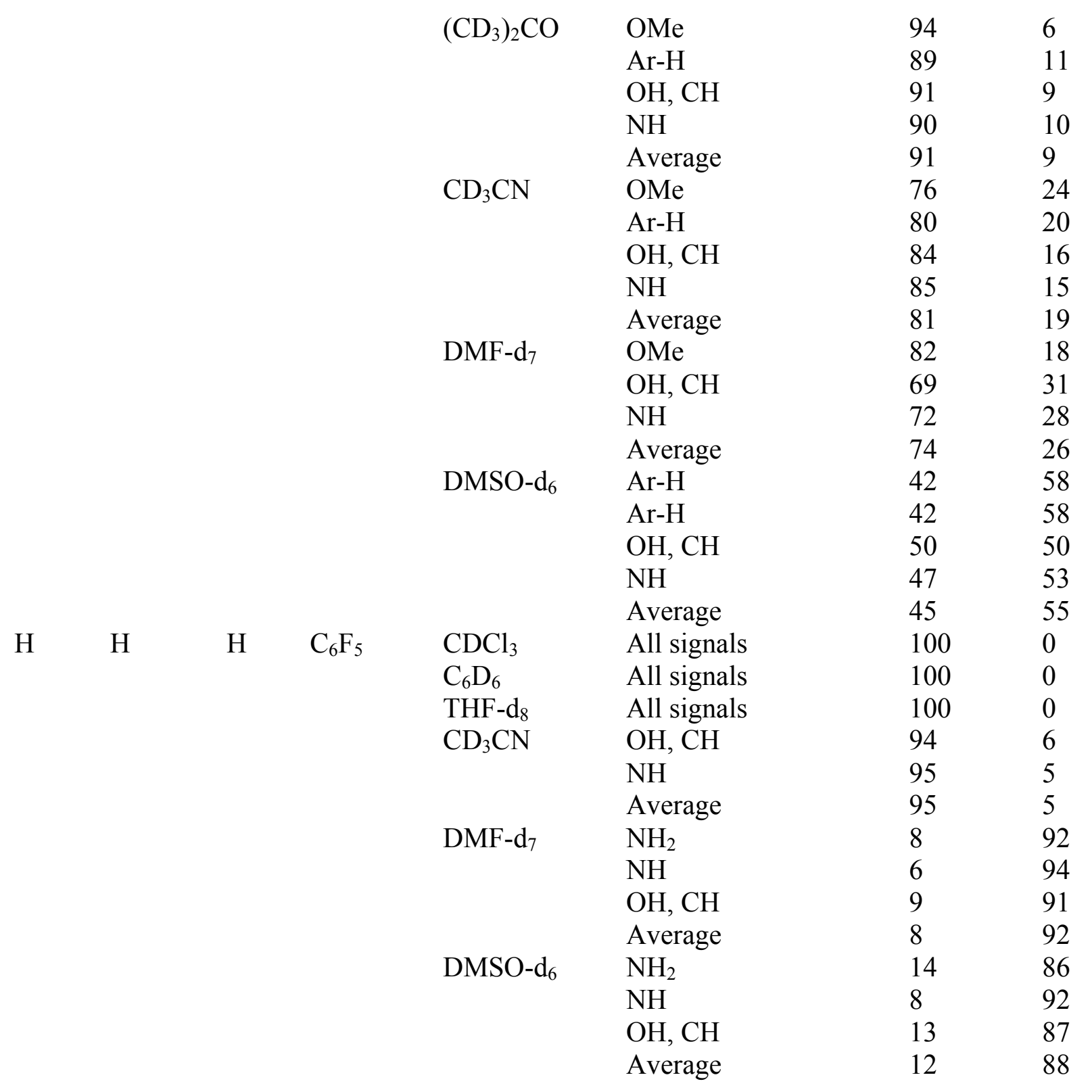




\begin{tabular}{|c|c|c|c|c|c|c|c|}
\hline \multirow[t]{15}{*}{$\mathrm{H}$} & $\mathrm{H}$ & $\mathrm{H}$ & $i$-Pr & $\mathrm{CDCl}_{3}$ & All signals & 100 & 0 \\
\hline & & & & $\mathrm{C}_{6} \mathrm{D}_{6}$ & All signals & 100 & 0 \\
\hline & & & & $\mathrm{THF}^{-\mathrm{d}_{8}}$ & All signals & 100 & 0 \\
\hline & & & & $\mathrm{CD}_{3} \mathrm{CN}$ & $\mathrm{CH}(i-\mathrm{Pr})$ & 84 & 16 \\
\hline & & & & & $\mathrm{OH}, \mathrm{CH}$ & 84 & 16 \\
\hline & & & & & Average & 84 & 16 \\
\hline & & & & DMF- $\mathrm{d}_{7}$ & $\operatorname{Me}(i-\operatorname{Pr})$ & 78 & 22 \\
\hline & & & & & $\mathrm{CH}(i-\mathrm{Pr})$ & 78 & 22 \\
\hline & & & & & $\mathrm{OH}, \mathrm{CH}$ & 81 & 19 \\
\hline & & & & & NH & 76 & 24 \\
\hline & & & & & Average & 78 & 22 \\
\hline & & & & DMSO- $_{6}$ & $\mathrm{Me}(i-\mathrm{Pr})$ & 32 & 68 \\
\hline & & & & & $\mathrm{CH}(i-\mathrm{Pr})$ & 40 & 60 \\
\hline & & & & & $\mathrm{OH}, \mathrm{CH}$ & 49 & 51 \\
\hline & & & & & Average & 40 & 60 \\
\hline \multirow[t]{18}{*}{$\mathrm{H}$} & $\mathrm{H}$ & $\mathrm{H}$ & $t-\mathrm{Bu}$ & $\mathrm{CDCl}_{\mathrm{e}}$ & All signals & 100 & 0 \\
\hline & & & & $\mathrm{C}_{6} \mathrm{D}_{6}$ & All signals & 100 & 0 \\
\hline & & & & THF- $\mathrm{d}_{8}$ & $\mathrm{t}-\mathrm{Bu}$ & 90 & 10 \\
\hline & & & & & $\mathrm{OH}, \mathrm{CH}$ & 96 & 4 \\
\hline & & & & & $\mathrm{NH}_{2}$ & 94 & 6 \\
\hline & & & & & $\mathrm{NH}$ & 97 & 3 \\
\hline & & & & & Average & 94 & 6 \\
\hline & & & & $\mathrm{CD}_{3} \mathrm{CN}$ & $t-\mathrm{Bu}$ & 38 & 62 \\
\hline & & & & & $\mathrm{NH}_{2}$ & 32 & 68 \\
\hline & & & & & $\mathrm{NH}$ & 33 & 67 \\
\hline & & & & & $\mathrm{OH}, \mathrm{CH}$ & 33 & 67 \\
\hline & & & & & Average & 34 & 66 \\
\hline & & & & DMF- $\mathrm{d}_{7}$ & $t-\mathrm{Bu}$ & 49 & 51 \\
\hline & & & & & $\mathrm{OH}, \mathrm{CH}$ & 46 & 54 \\
\hline & & & & & Average & 47 & 53 \\
\hline & & & & $\mathrm{DMSO}^{-\mathrm{d}_{6}}$ & $t-\mathrm{Bu}$ & 33 & 67 \\
\hline & & & & & NH & 33 & 67 \\
\hline & & & & & Average & 33 & 67 \\
\hline
\end{tabular}


${ }^{a}$ Signals used for determining the Enol/Amide ratio.

Table S2. ${ }^{1} \mathrm{H}$ NMR Spectral Data of $\mathrm{R}^{3} \mathrm{NHCOCH}(\mathrm{CN}) \mathrm{CONR}^{1} \mathrm{R}^{2} / \mathrm{R}^{3} \mathrm{NHC}(\mathrm{OH})=\mathrm{C}(\mathrm{CN}) \mathrm{CONR}{ }^{1} \mathrm{R}^{2} / \mathrm{R}^{3} \mathrm{NHCOC}(\mathrm{CN})=\mathrm{C}(\mathrm{OH}) \mathrm{NR}{ }^{1} \mathrm{R}^{2}(\mathbf{4} / \mathbf{5} / \mathbf{6})$ System in Several Solvents ${ }^{\mathrm{a}, \mathrm{b}}$

\begin{tabular}{|c|c|c|c|c|c|c|c|c|}
\hline \multirow{2}{*}{$\begin{array}{l}\mathbf{R}^{3} \\
\mathrm{C}_{6} \mathrm{H}_{5}\end{array}$} & \multirow{2}{*}{$\begin{array}{c}\mathbf{R}^{1} \\
\mathrm{CH}_{3}\end{array}$} & \multirow{2}{*}{$\begin{array}{l}\mathbf{R}^{2} \\
\mathrm{CH}_{3}\end{array}$} & \multirow{2}{*}{$\begin{array}{ll}\text { Solvent } & \mathbf{A} \\
& \text { or Io } \\
\mathrm{CDCl}_{3} & \end{array}$} & \multicolumn{2}{|c|}{ 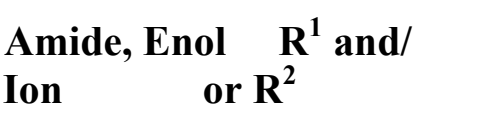 } & $\mathbf{R}^{3}$ & \multicolumn{2}{|c|}{$\begin{array}{c}\mathbf{C H}, \mathbf{O H} \\
\text { or } \mathbf{H}^{+}\end{array}$} \\
\hline & & & & Enol & 3.23 & $\begin{array}{l}7.13(\mathrm{t}, \mathrm{J}=7.3 \mathrm{~Hz}) ; 7.33(\mathrm{t}, \mathrm{J}=8.3 \mathrm{~Hz}) \\
7.43(\mathrm{~d}, \mathrm{~J}=7.9 \mathrm{~Hz})\end{array}$ & 7.67 (br) & 17.79 \\
\hline & & & & Amide & $3.02 ; 3.18$ & $\begin{array}{l}7.13(\mathrm{t}, \mathrm{ov}) ; 7.33(\mathrm{t}, \mathrm{ov}) ; 7.53(\mathrm{~d}, \mathrm{~J}= \\
7.7 \mathrm{~Hz})\end{array}$ & 9.16 (br) & 4.80 \\
\hline & & & & Amide & $2.20 ; 2.26$ & $\begin{array}{l}6.87(\mathrm{t}, \mathrm{ov}) ; 7.05(\mathrm{t}, \mathrm{ov}) ; 7.57(\mathrm{~d}, \mathrm{~J}= \\
7.6 \mathrm{~Hz})\end{array}$ & 9.49 & 4.32 \\
\hline & & & THF- $\mathrm{d}_{8}$ & Enol & 3.17 & $\begin{array}{l}7.07(\mathrm{t}, \mathrm{J}=7.3 \mathrm{~Hz}) ; 7.27(\mathrm{t}, \mathrm{J}=8.0 \mathrm{~Hz}) \\
7.49(\mathrm{~d}, \mathrm{~J}=7.9 \mathrm{~Hz})\end{array}$ & 8.52 & 18.18 \\
\hline & & & & Amide & $2.96 ; 3.14$ & $\begin{array}{l}7.07(\mathrm{t}, \mathrm{ov}) ; 7.27(\mathrm{t}, \mathrm{ov}) ; 7.60(\mathrm{~d}, \mathrm{~J}= \\
8.0 \mathrm{~Hz})\end{array}$ & 9.64 & 5.05 \\
\hline & & & & Amide & $2.97 ; 3.16$ & $\begin{array}{l}7.13(\mathrm{t}, \mathrm{J}=7.4 \mathrm{~Hz}) ; 7.33(\mathrm{t}, \mathrm{J}=8.1 \mathrm{~Hz}) \\
7.62(\mathrm{~d}, \mathrm{~J}=7.8 \mathrm{~Hz})\end{array}$ & 9.66 & 5.31 \\
\hline
\end{tabular}




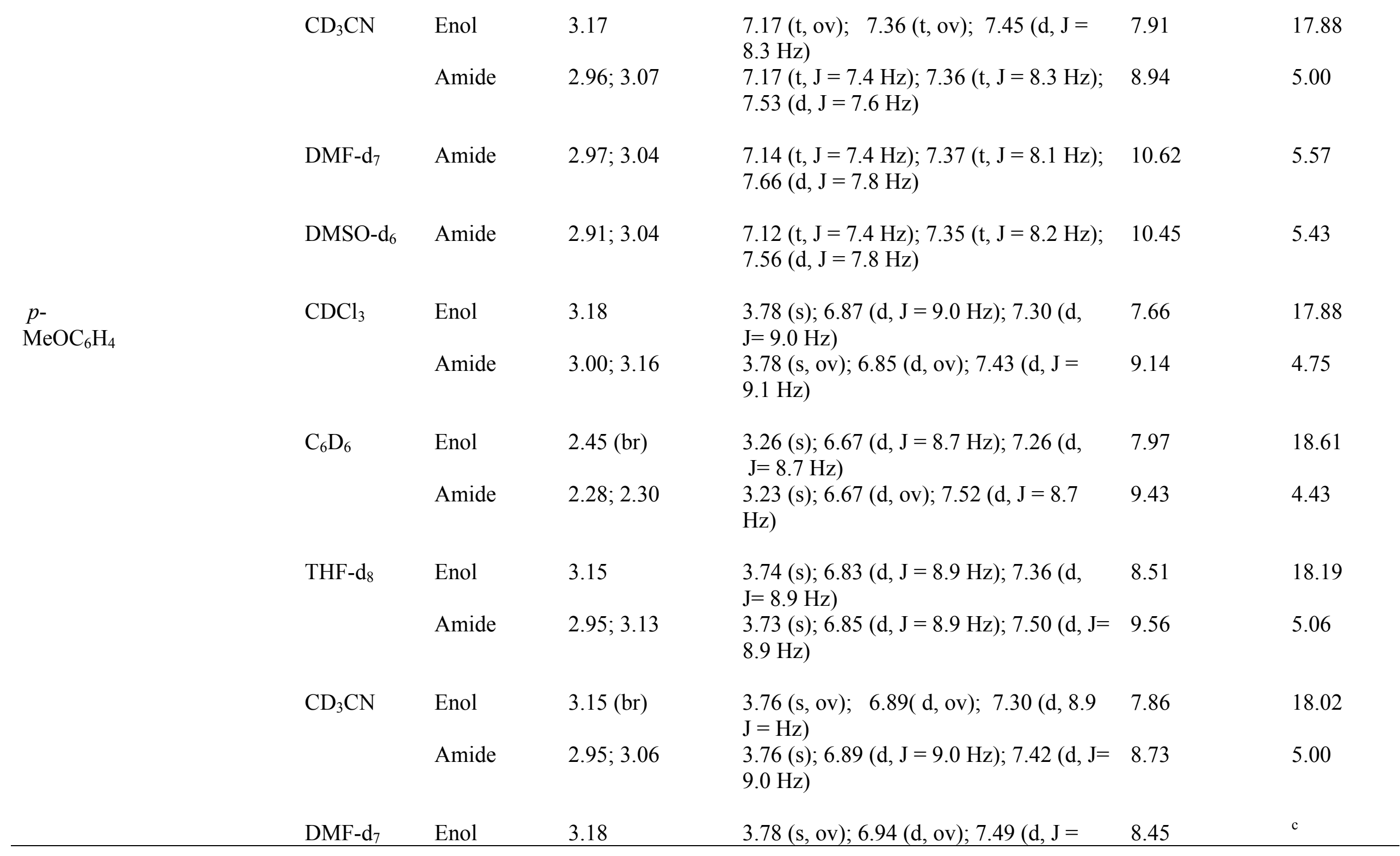




\begin{tabular}{|c|c|c|c|c|c|c|}
\hline & & Amide & $2.95 ; 3.14$ & $\begin{array}{l}8.7 \mathrm{~Hz}) \\
3.78(\mathrm{~s}) ; 6.94(\mathrm{~d}, \mathrm{~J}=8.7 \mathrm{~Hz}) ; 7.57(\mathrm{~d}, \mathrm{~J}= \\
8.7 \mathrm{~Hz})\end{array}$ & 10.48 & 5.51 \\
\hline & DMSO-d ${ }_{6}$ & Amide & $2.90 ; 3.03$ & $\begin{array}{l}3.73(\mathrm{~s}) ; 6.92(\mathrm{~d}, \mathrm{~J}=9.0 \mathrm{~Hz}) ; 7.46(\mathrm{~d}, \mathrm{~J}= \\
8.7 \mathrm{~Hz})\end{array}$ & 10.34 & 5.39 \\
\hline \multirow[t]{5}{*}{$\mathrm{C}_{6} \mathrm{~F}_{5}$} & $\mathrm{CDCl}_{3}$ & $\begin{array}{l}\text { Enol } \\
\text { Amide }\end{array}$ & $\begin{array}{l}3.23(\mathrm{br}) \\
3.04 ; 3.22\end{array}$ & & $\begin{array}{l}7.44 \\
9.40\end{array}$ & $\begin{array}{l}16.82 \\
4.84\end{array}$ \\
\hline & $\mathrm{C}_{6} \mathrm{D}_{6}$ & $\begin{array}{l}\text { Enol } \\
\text { Amide }\end{array}$ & $\begin{array}{l}2.29(\mathrm{br}) \\
2.07 ; 2.23\end{array}$ & & $\begin{array}{l}7.65 \\
9.37\end{array}$ & $\begin{array}{l}17.46 \\
4.10\end{array}$ \\
\hline & $\begin{array}{l}\mathrm{THF}-\mathrm{d}_{8} \\
\mathrm{CD}_{3} \mathrm{CN}\end{array}$ & $\begin{array}{l}\text { Enol } \\
\text { Amide } \\
\text { Enol } \\
\text { Amide }\end{array}$ & $\begin{array}{l}3.20(\mathrm{br}) \\
2.98 ; 3.14 \\
3.19 \\
2.98 ; 3.09\end{array}$ & & $\begin{array}{l}8.81 \\
9.85 \\
7.76 \\
8.76\end{array}$ & $\begin{array}{l}17.46 \\
5.26 \\
16.95 \\
5.15\end{array}$ \\
\hline & DMF-d 7 & $\begin{array}{l}\text { Enol } \\
\text { Amide }\end{array}$ & $\begin{array}{l}3.24 \\
2.98 ; 3.19\end{array}$ & & $\begin{array}{l}9.81 \\
10.89\end{array}$ & $\begin{array}{l}17.39(\mathrm{br}) \\
5.83\end{array}$ \\
\hline & $\mathrm{DMSO}^{-\mathrm{d}_{6}}$ & $\begin{array}{l}\text { Enol } \\
\text { Amide }\end{array}$ & $\begin{array}{l}3.14 \\
2.92 ; 3.06\end{array}$ & & $\begin{array}{l}9.91 \\
10.73\end{array}$ & $\begin{array}{l}17.28(\mathrm{br}) \\
5.69\end{array}$ \\
\hline \multirow[t]{4}{*}{$\mathrm{CHMe}_{2}$} & $\mathrm{CDCl}_{3}$ & Enol & 3.15 & $\begin{array}{l}1.12(\mathrm{~d}, \mathrm{~J}=6.5 \mathrm{~Hz}) ; 3.84 \text { (octet, } \mathrm{J}=6.4 \\
\mathrm{~Hz}) ; 1.19(\mathrm{~d}, \mathrm{~J}=6.6 \mathrm{~Hz}) ; 4.09 \text { (octet, J= } \\
6.6 \mathrm{~Hz})\end{array}$ & 5.62 & 18.18 \\
\hline & & Amide & $3.02 ; 3.17$ & $\begin{array}{l}1.18(\mathrm{~d}, \mathrm{~J}=6.6 \mathrm{~Hz}) ; 1.20(\mathrm{~d}, \mathrm{~J}=6.7 \\
\mathrm{Hz}) ; 4.09 \text { (octet, ov) }\end{array}$ & 6.68 & 4.61 \\
\hline & $\mathrm{C}_{6} \mathrm{D}_{6}$ & Enol & 2.49 & $\begin{array}{l}0.80(\mathrm{~d}, \mathrm{~J}=6.5 \mathrm{~Hz}) ; 3.98 \text { (octet, } \mathrm{J}=6.7 \\
\mathrm{~Hz})\end{array}$ & 5.89 & 19.02 \\
\hline & & Amide & $2.29 ; 2.35$ & $0.80(\mathrm{~d}, \mathrm{ov}) ; 3.98$ (octet, ov) & 6.92 & 4.28 \\
\hline
\end{tabular}




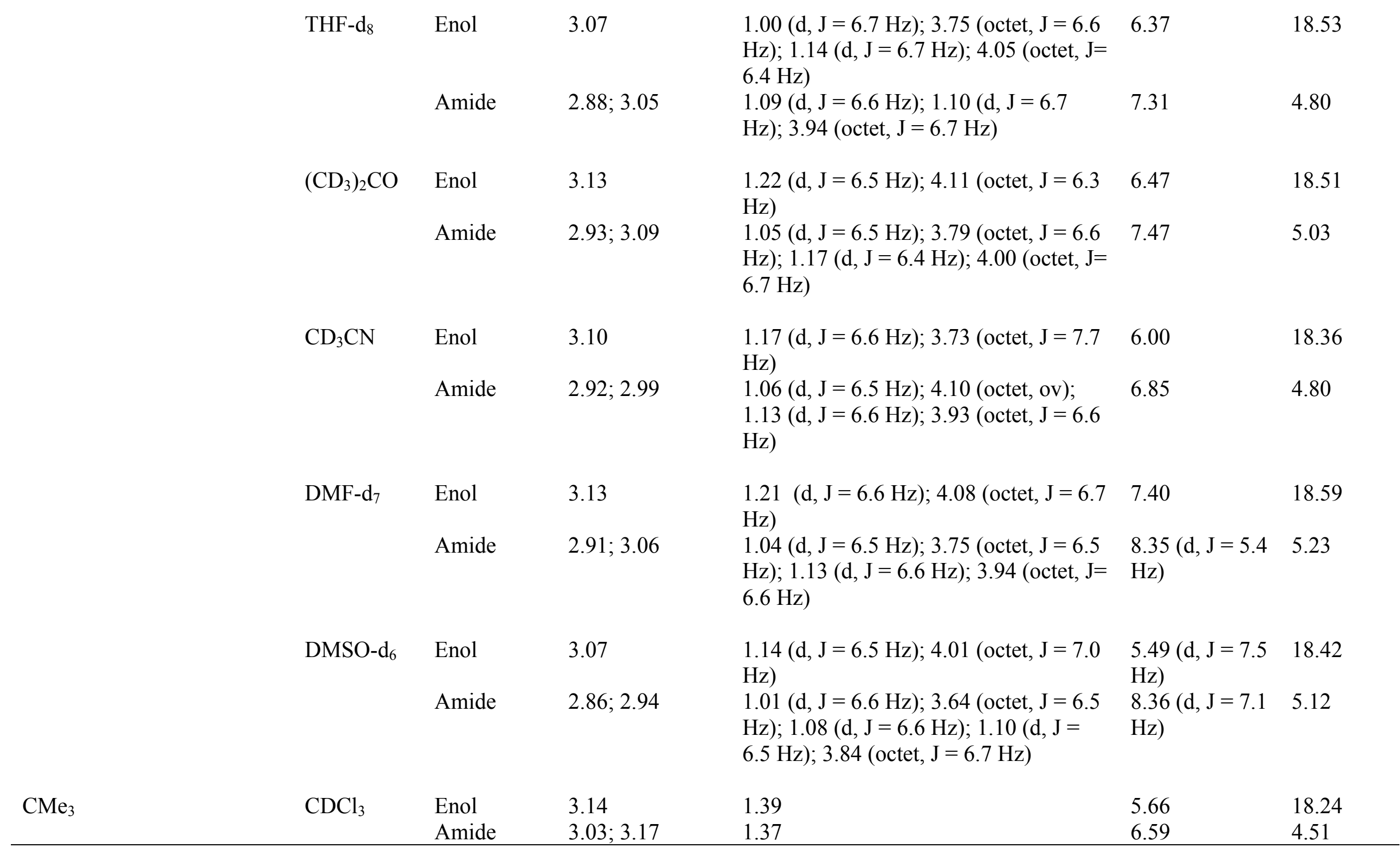




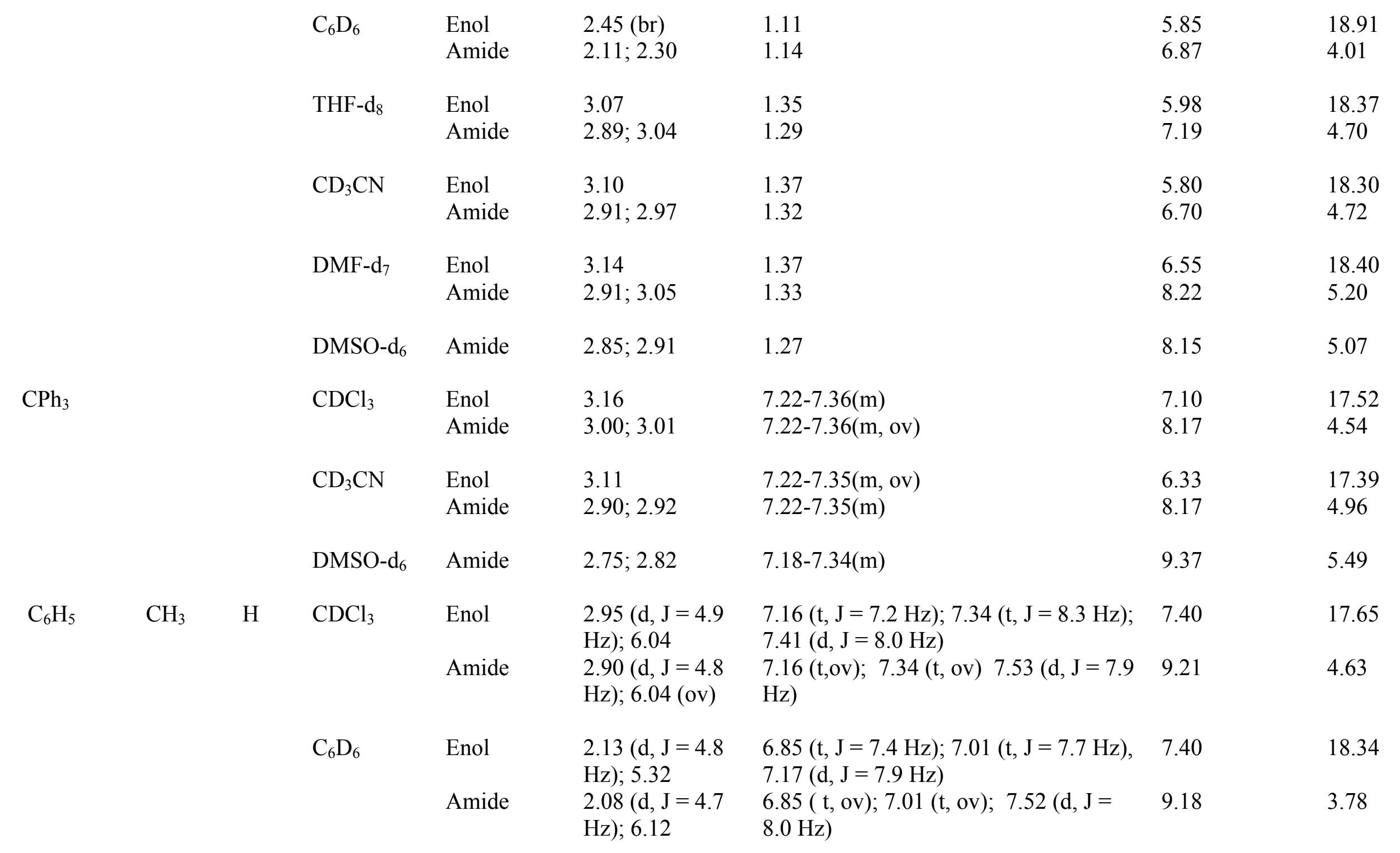




\begin{tabular}{|c|c|c|c|c|c|c|}
\hline & THF- $\mathrm{d}_{8}$ & Enol & $2.86(\mathrm{~d}, \mathrm{~J}=4.0$ & $7.05(\mathrm{t}, \mathrm{J}=6.6 \mathrm{~Hz}) ; 7.25(\mathrm{t}, \mathrm{J}=7.8 \mathrm{~Hz})$ & 8.58 & 18.18 \\
\hline & & Amide & $\begin{array}{l}\mathrm{Hz}) ; 7.32(\mathrm{br}) \\
2.77(\mathrm{~d}, \mathrm{~J}=4.5 \\
\mathrm{Hz}) ; 7.77(\mathrm{br})\end{array}$ & $\begin{array}{l}7.48(\mathrm{~d}, \mathrm{~J}=7.7 \mathrm{~Hz}) \\
7.05(\mathrm{t}, \mathrm{ov}) ; 7.25(\mathrm{t}, \mathrm{ov}) ; 7.58(\mathrm{~d}, \mathrm{~J}= \\
7.6 \mathrm{~Hz})\end{array}$ & 9.68 & 4.60 \\
\hline & $\mathrm{CD}_{3} \mathrm{CN}$ & Enol & $\begin{array}{l}2.85(\mathrm{~d}, \mathrm{~J}=3.7 \\
\mathrm{Hz}) ; 6.51\end{array}$ & $\begin{array}{l}7.16(\mathrm{t}, \mathrm{J}=7.2 \mathrm{~Hz}) ; 7.35(\mathrm{t}, \mathrm{J}=7.6 \mathrm{~Hz}) \\
7.42((\mathrm{~d}, \mathrm{~J}=7.8 \mathrm{~Hz})\end{array}$ & 7.87 & 17.89 \\
\hline & & Amide & $\begin{array}{l}2.78(\mathrm{~d}, \mathrm{~J}=4.0 \\
\mathrm{Hz}) ; 7.00\end{array}$ & $\begin{array}{l}7.16(\mathrm{t}, \mathrm{ov}) ; 7.35(\mathrm{t}, \mathrm{ov}) ; 7.54(\mathrm{~d}, \mathrm{~J}=7.7 \\
\mathrm{Hz})\end{array}$ & 8.95 & 4.65 \\
\hline & DMF-d & Enol & $2.90 ; 8.05$ & $\begin{array}{l}7.12(\mathrm{t}, \mathrm{ov}) ; 7.35(\mathrm{t}, \mathrm{ov}) ; 7.61(\mathrm{~d}, \mathrm{~J}= \\
7.7 \mathrm{~Hz})\end{array}$ & 9.59 & 18.21 \\
\hline & & Amide & $\begin{array}{l}2.78(\mathrm{~d}, \mathrm{~J}=4.0 \\
\mathrm{Hz}) ; 8.38\end{array}$ & $\begin{array}{l}7.12(\mathrm{t}, \mathrm{J}=7.0 \mathrm{~Hz}) ; 7.35(\mathrm{t}, \mathrm{J}=6.7 \mathrm{~Hz}) \\
7.66(\mathrm{~d}, \mathrm{~J}=7.7 \mathrm{~Hz})\end{array}$ & 10.53 & 5.08 \\
\hline & $\mathrm{DMSO}_{-} \mathrm{d}_{6}$ & Enol & $2.77 ; 8.33$ (ov) & $\begin{array}{l}7.12(\mathrm{t}, \mathrm{ov}) ; 7.35(\mathrm{t}, \mathrm{ov}) ; 7.45(\mathrm{~d}, \mathrm{~J}= \\
7.3 \mathrm{~Hz})\end{array}$ & 9.61 & 18.16 \\
\hline & & Amide & $\begin{array}{l}2.68(\mathrm{~d}, \mathrm{~J}=3.2 \\
\mathrm{Hz}) ; 8.33\end{array}$ & $\begin{array}{l}7.12(\mathrm{t}, \mathrm{J}=6.0 \mathrm{~Hz}) ; 7.35(\mathrm{t}, \mathrm{J}=7.2 \mathrm{~Hz}) \\
7.56(\mathrm{~d}, \mathrm{~J}=7.3 \mathrm{~Hz})\end{array}$ & 10.38 & 4.90 \\
\hline $\begin{array}{l}p- \\
\mathrm{MeOC}_{6} \mathrm{H}_{4}\end{array}$ & $\mathrm{CDCl}_{3}$ & Enol & $2.96 ; 5.76$ & $\begin{array}{l}3.80(\mathrm{~s}) ; 6.88(\mathrm{~d}, \mathrm{~J}=8.9 \mathrm{~Hz}) ; 7.29(\mathrm{~d}, \\
\mathrm{J}=8.9 \mathrm{~Hz})\end{array}$ & 7.16 & 17.71 \\
\hline & & Amide & $2.92 ; 7.23$ & $\begin{array}{l}3.80(\mathrm{~s}, \mathrm{ov}) ; 6.88(\mathrm{~d}, \mathrm{ov}) ; 7.42(\mathrm{~d} \\
\mathrm{J}=8.9 \mathrm{~Hz})\end{array}$ & 8.96 & 4.56 \\
\hline & $\mathrm{C}_{6} \mathrm{D}_{6}$ & Enol & $2.06 ; 5.00$ & $\begin{array}{l}3.22(\mathrm{~s}) ; 6.61(\mathrm{~d}, \mathrm{~J}=8.9 \mathrm{~Hz}) ; 6.96(\mathrm{~d}, \mathrm{~J}= \\
8.9 \mathrm{~Hz})\end{array}$ & 7.08 & 18.47 \\
\hline & & Amide & $2.02 ; 5.85$ & $\begin{array}{l}3.20(\mathrm{~s}) ; 6.72(\mathrm{~d}, \mathrm{~J}=8.6 \mathrm{~Hz}) ; 7.40(\mathrm{~d}, \mathrm{~J}= \\
8.8 \mathrm{~Hz})\end{array}$ & 7.36 & 5.60 \\
\hline & $\mathrm{CD}_{2} \mathrm{Cl}_{2}$ & Enol & $2.84 ; 5.68$ & $\begin{array}{l}3.70(\mathrm{~s}) ; 6.79(\mathrm{~d}, \mathrm{~J}=7.9 \mathrm{~Hz}) ; 7.20(\mathrm{~d}, \mathrm{~J}= \\
8.9 \mathrm{~Hz})\end{array}$ & 7.09 & 17.7 \\
\hline & & Amide & 2.80 & $\begin{array}{l}3.70(\mathrm{~s}, \text { ov }) ; 6.79(\mathrm{~d}, \text { ov }) ; 7.32(\mathrm{~d}, \mathrm{~J}= \\
8.9 \mathrm{~Hz})\end{array}$ & 8.85 & 4.49 \\
\hline
\end{tabular}




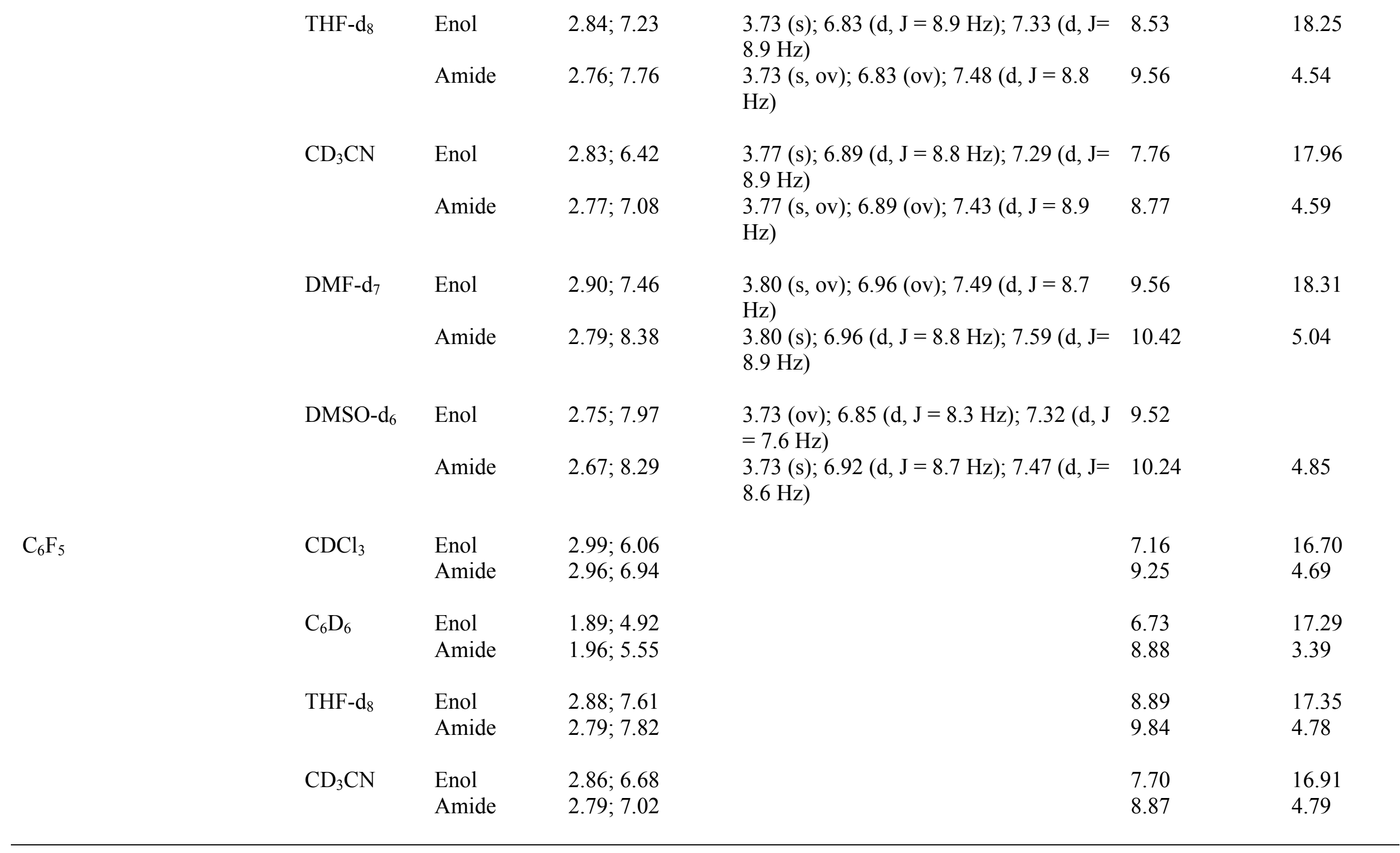




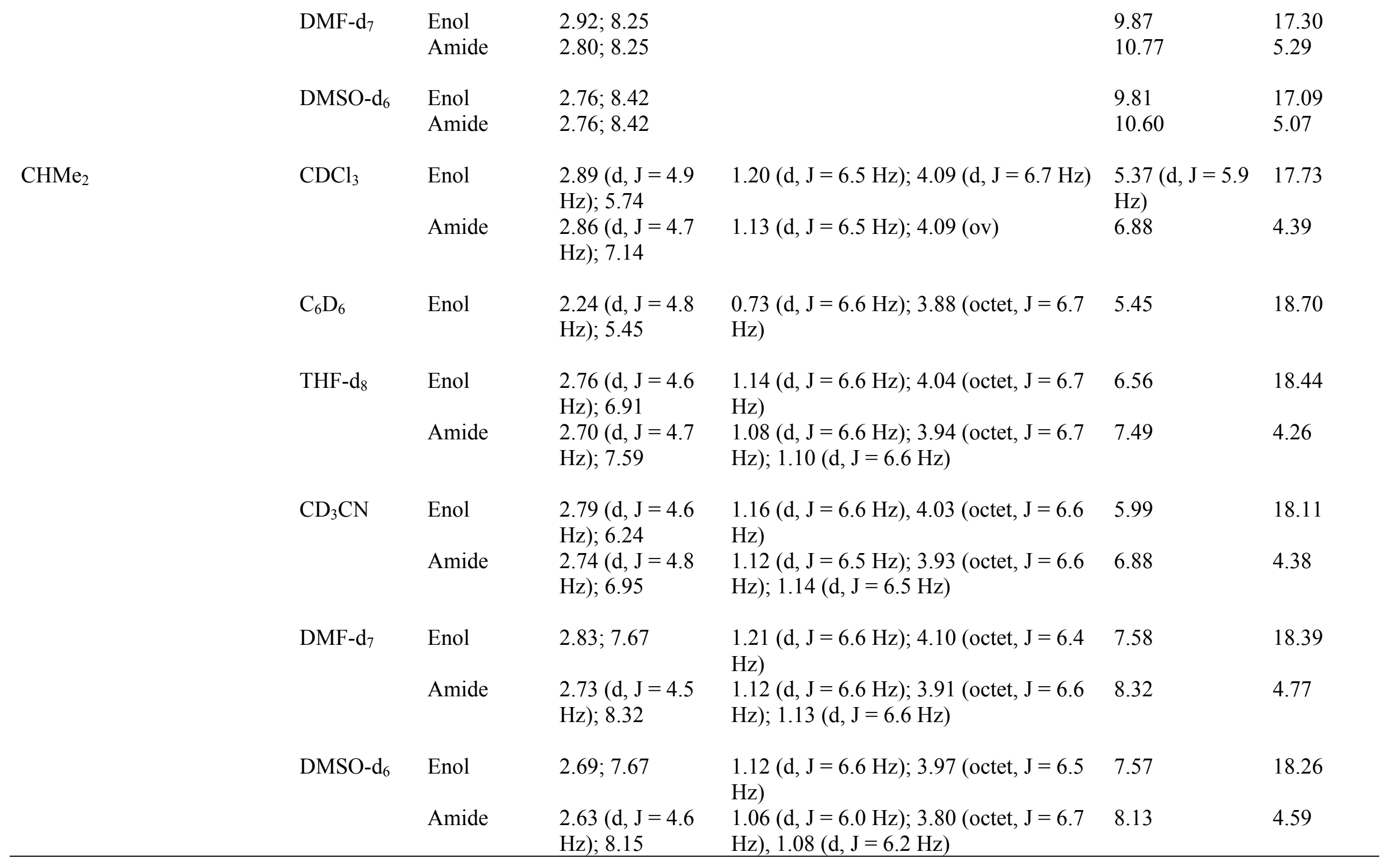




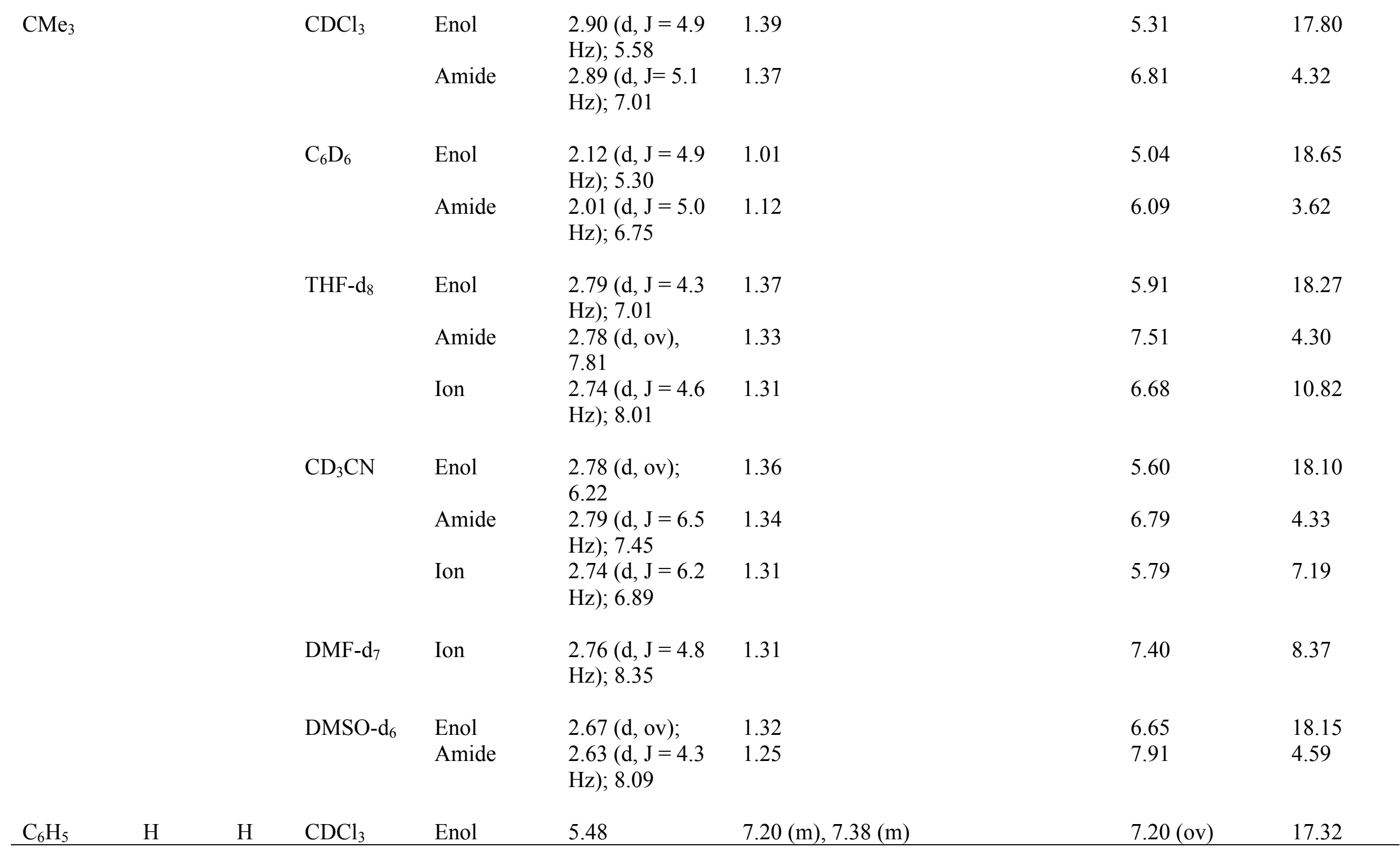




\begin{tabular}{|c|c|c|c|c|c|c|}
\hline & $\mathrm{C}_{6} \mathrm{D}_{6}$ & Enol & $4.01 ; 6.86$ & $6.93(\mathrm{~m})$ & 7.36 & 17.70 \\
\hline & \multirow[t]{2}{*}{ THF- $\mathrm{d}_{8}$} & Enol & 7.39 (br) & $\begin{array}{l}7.04(\mathrm{t}, \mathrm{J}=7.3 \mathrm{~Hz}) ; 7.25(\mathrm{t}, \mathrm{J}=7.9 \mathrm{~Hz}) \\
7.50(\mathrm{~d}, \mathrm{~J}=7.9 \mathrm{~Hz})\end{array}$ & 8.53 & 17.35 \\
\hline & & Amide & 4.72 (br) & $\begin{array}{l}7.04(\mathrm{t}, \mathrm{ov}) ; 7.25(\mathrm{t}, \mathrm{ov}) ; 7.59(\mathrm{~d}, \mathrm{~J}= \\
7.9 \mathrm{~Hz})\end{array}$ & 9.66 & 4.58 \\
\hline & \multirow[t]{2}{*}{$\mathrm{CD}_{3} \mathrm{CN}$} & Enol & $6.36(\mathrm{br})$ & $\begin{array}{l}7.17(\mathrm{t}, \mathrm{J}=7.2 \mathrm{~Hz}) ; 7.35(\mathrm{t}, \mathrm{J}=8.1 \mathrm{~Hz}) \\
7.43(\mathrm{~d}, \mathrm{~J}=7.7 \mathrm{~Hz})\end{array}$ & 7.88 & 17.29 \\
\hline & & Amide & 6.71 & $\begin{array}{l}7.17(\mathrm{t}, \mathrm{ov}) ; 7.35(\mathrm{t}, \mathrm{ov}) ; 7.53(\mathrm{~d}, \mathrm{~J}= \\
7.8 \mathrm{~Hz})\end{array}$ & 8.85 & 4.64 \\
\hline & \multirow[t]{2}{*}{ DMF-d 7} & Enol & $3.51(\mathrm{br})$ & $\begin{array}{l}7.11(\mathrm{t}, \mathrm{J}=6.9 \mathrm{~Hz}) ; 7.3(\mathrm{t}, \mathrm{J}=7.3 \mathrm{~Hz}) \\
7.62(\mathrm{~d}, \mathrm{~J}=7.6 \mathrm{~Hz})\end{array}$ & 9.56 & 17.41 \\
\hline & & Amide & 8.28 (br) & $7.11(\mathrm{t}, \mathrm{ov}) ; 7.3(\mathrm{t}, \mathrm{ov}) ; 7.62(\mathrm{~d}, \mathrm{ov})$ & 10.54 & 5.07 \\
\hline & \multirow{2}{*}{$\mathrm{DMSO}_{-} \mathrm{d}_{6}$} & Enol & 7.9 (br) & $7.10(\mathrm{~m}) ; 7.32(\mathrm{~m}) ; 7.47(\mathrm{~d}, \mathrm{~J}=7.7 \mathrm{~Hz})$ & 9.53 & $17.00(\mathrm{br})$ \\
\hline & & Amide & $7.73 ; 7.83$ & $7.10(\mathrm{~m}) ; 7.32(\mathrm{~m}) ; 7.55(\mathrm{~d}, \mathrm{~J}=7.8 \mathrm{~Hz})$ & 10.36 & 4.68 \\
\hline \multirow[t]{6}{*}{$\begin{array}{l}p-\mathrm{MeO}- \\
\mathrm{C}_{6} \mathrm{H}_{4}\end{array}$} & \multirow[t]{2}{*}{ THF- $\mathrm{d}_{8}$} & Enol & 7.23 & $\begin{array}{l}3.73(\mathrm{~s}) ; 6.81(\mathrm{~d}, \mathrm{~J}=8.8 \mathrm{~Hz}) ; 7.36(\mathrm{~d}, \mathrm{~J}= \\
8.7 \mathrm{~Hz})\end{array}$ & 8.48 & 17.54 \\
\hline & & Amide & c & $3.70(\mathrm{~s}) ; 6.81(\mathrm{ov}) ; 7.49(\mathrm{~d}, \mathrm{~J}=7.8 \mathrm{~Hz})$ & 10.84 & 4.73 \\
\hline & \multirow[t]{2}{*}{$\mathrm{CD}_{3} \mathrm{CN}$} & Enol & 6.29 & $\begin{array}{l}3.77(\mathrm{~s}) ; 6.89(\mathrm{~d}, \mathrm{~J}=8.9 \mathrm{~Hz}) ; 7.30(\mathrm{~d} \\
\mathrm{J}=8.9 \mathrm{~Hz})\end{array}$ & 7.81 & 17.43 \\
\hline & & Amide & 7.06 & $\begin{array}{l}3.75(\mathrm{~s}) ; 6.86(\mathrm{~d}, \mathrm{~J}=9.2 \mathrm{~Hz}) ; 7.43(\mathrm{~d}, \mathrm{~J}= \\
8.8 \mathrm{~Hz})\end{array}$ & 8.73 & 4.61 \\
\hline & \multirow[t]{2}{*}{$\left(\mathrm{CD}_{3}\right)_{2} \mathrm{CO}$} & Enol & 7.23 & $\begin{array}{l}3.78(\mathrm{~s}) ; 6.89(\mathrm{~d}, \mathrm{~J}=9.0 \mathrm{~Hz}) ; 7.4(\mathrm{~d}, \\
\mathrm{J}=8.9 \mathrm{~Hz})\end{array}$ & 8.52 & 17.54 \\
\hline & & Amide & $7.23(\mathrm{~s}, \mathrm{ov})$ & $\begin{array}{l}3.74(\mathrm{~s}) ; 6.89(\mathrm{~d}, \mathrm{ov}) ; 7.55(\mathrm{~d}, \mathrm{~J}=9.0 \\
\mathrm{Hz})\end{array}$ & 9.55 & 4.82 \\
\hline
\end{tabular}




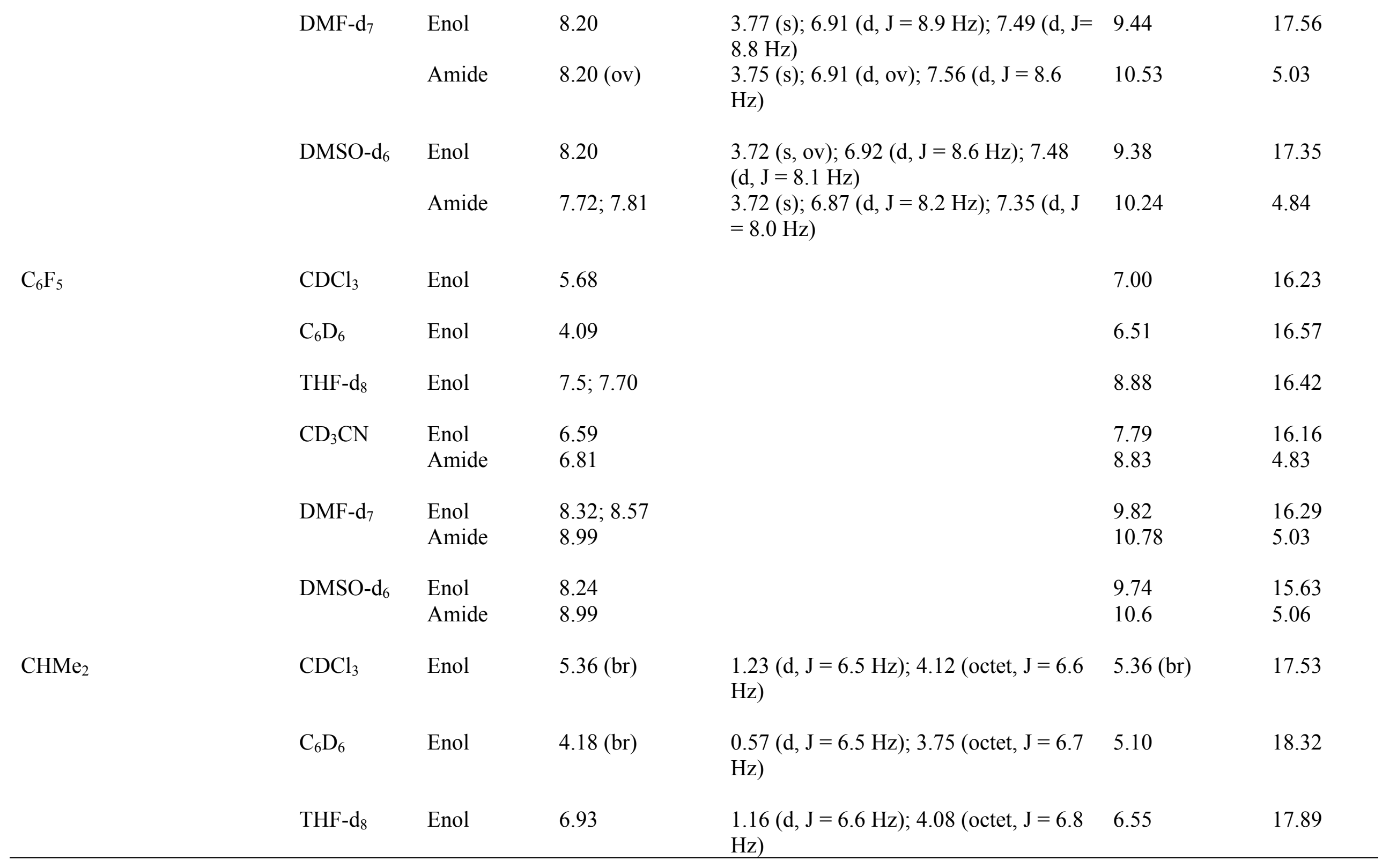




\begin{tabular}{|c|c|c|c|c|c|c|c|c|}
\hline & & & $\mathrm{CD}_{3} \mathrm{CN}$ & Enol & 6.08 & $\begin{array}{l}1.16(\mathrm{~d}, \mathrm{~J}=6.6 \mathrm{~Hz}) ; 4.05 \text { (octet, } \mathrm{J}=6.4 \\
\mathrm{~Hz})\end{array}$ & 5.90 & 17.80 \\
\hline & & & & Amide & 6.80 & $1.11(\mathrm{~d}$, ov); 3.93 (octet, $\mathrm{J}=6.7 \mathrm{~Hz})$ & 6.67 & 4.38 \\
\hline & & & DMF-d 7 & Enol & $7.48 ; 7.69$ & $\begin{array}{l}1.21(\mathrm{~d}, \mathrm{~J}=6.6 \mathrm{~Hz}) ; 4.10 \text { (octet, } \mathrm{J}= \\
6.60 \mathrm{~Hz})\end{array}$ & 7.79 & 18.00 \\
\hline & & & & Amide & $\begin{array}{l}7.43(\mathrm{ov}) \\
7.69(\mathrm{ov})\end{array}$ & $\begin{array}{l}1.13(\mathrm{~d}, \mathrm{~J}=5.7 \mathrm{~Hz}) ; 1.15(\mathrm{~d}, \mathrm{~J}=5.9 \\
\mathrm{Hz}) ; 3.93 \text { (octet, } \mathrm{J}=6.6 \mathrm{~Hz})\end{array}$ & 8.19 & 4.80 \\
\hline & & & DMSO-d $_{6}$ & Enol & $\mathrm{c}$ & $\begin{array}{l}1.06(\mathrm{~d}, \mathrm{~J}=6.6 \mathrm{~Hz}) ; 3.81 \text { (octet, } \mathrm{J}=6.5 \\
\mathrm{~Hz})\end{array}$ & $\begin{array}{l}8.13(\mathrm{~d}, \mathrm{~J}=7.3 \\
\mathrm{Hz})\end{array}$ & $17.76(\mathrm{br})$ \\
\hline & & & & Amide & $3.36(\mathrm{br})$ & $\begin{array}{l}1.10(\mathrm{~d}, \mathrm{~J}=6.5 \mathrm{~Hz}) ; 3.99 \text { (octet, } \mathrm{J}=6.6 \\
\mathrm{~Hz})\end{array}$ & 7.60 & 4.59 \\
\hline $\mathrm{CMe}_{3}$ & & & $\mathrm{CDCl}_{3}$ & Enol & 5.43 & 1.40 & 5.43 & 17.59 \\
\hline & & & $\mathrm{C}_{6} \mathrm{D}_{6}$ & Enol & 4.17 & 0.96 & 5.26 & 18.25 \\
\hline & & & THF- $\mathrm{d}_{8}$ & Enol & 6.96 & 1.34 & 5.87 & 17.61 \\
\hline & & & & Amide & 7.35 & 1.29 & 7.30 & 4.78 \\
\hline & & & $\mathrm{CD}_{3} \mathrm{CN}$ & Enol & 6.12 & 1.37 & 5.68 & 17.71 \\
\hline & & & & Amide & 6.67 & 1.32 & 6.27 & 4.37 \\
\hline & & & DMF-d ${ }_{7}$ & Enol & 7.87 & 1.39 & 6.52 & 17.96 \\
\hline & & & & Amide & 7.68 & 1.32 & 7.77 & 4.78 \\
\hline & & & DMSO-d $_{6}$ & Enol & 7.68 (br) & 1.32 & $6.51(\mathrm{br})$ & $17.53(\mathrm{br})$ \\
\hline & & & & Amide & 7.56 & 1.26 & 7.89 & 4.58 \\
\hline $\mathrm{C}_{6} \mathrm{H}_{5}$ & $\mathrm{C}_{6} \mathrm{~F}_{5}$ & $\mathrm{H}$ & $\mathrm{CDCl}_{3}$ & Enol & 7.28 & $7.28(\mathrm{~m}) ; 7.42(\mathrm{~m})$ & 7.60 & 17.52 \\
\hline
\end{tabular}




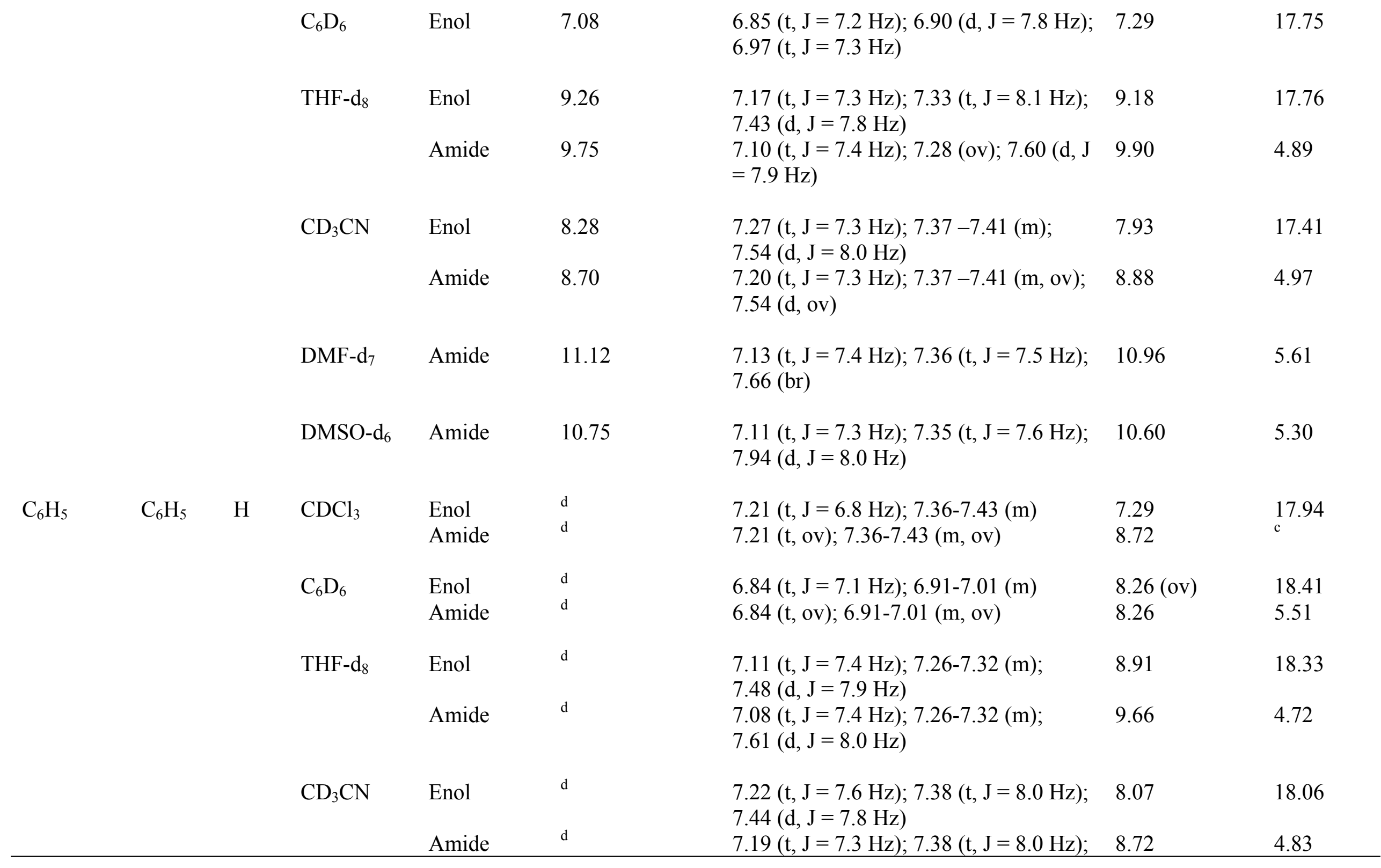




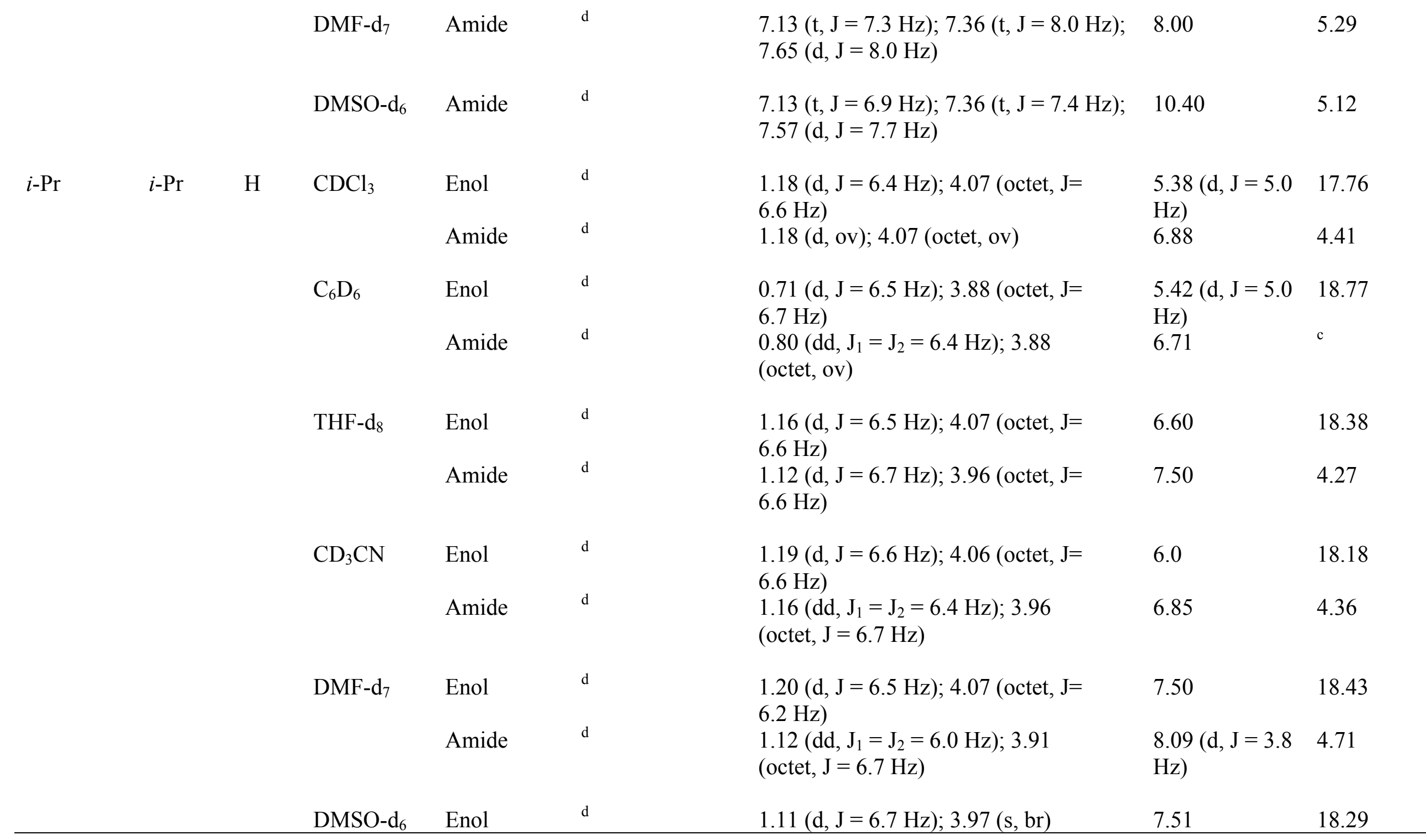




\begin{tabular}{|c|c|c|c|c|c|c|c|c|}
\hline & & & & Amide & d & $\begin{array}{l}1.04(\mathrm{~d}, \mathrm{~J}=6.5 \mathrm{~Hz}) ; 1.08(\mathrm{~d}, \mathrm{~J}= \\
6.5 \mathrm{~Hz}) ; 3.81 \text { (octet, } \mathrm{J}=6.6 \mathrm{~Hz})\end{array}$ & $\begin{array}{l}8.04(\mathrm{~d}, \mathrm{~J}=6.6 \\
\mathrm{Hz})\end{array}$ & 4.54 \\
\hline \multirow[t]{8}{*}{$\mathrm{CHPh}_{2}$} & $\mathrm{CHPh}_{2}$ & $\mathrm{H}$ & $\mathrm{CDCl}_{3}$ & Enol & d & $6.20(\mathrm{~d}, \mathrm{~J}=7.8 \mathrm{~Hz}) ; 7.16-7.37(\mathrm{~m})$ & $\begin{array}{l}6.04(\mathrm{~d}, \mathrm{~J}=7.6 \\
\mathrm{Hz})\end{array}$ & 17.69 \\
\hline & & & $\mathrm{C}_{6} \mathrm{D}_{6}$ & Enol & $\mathrm{d}$ & $6.24(\mathrm{~d}, \mathrm{~J}=8.1 \mathrm{~Hz}) ; 6.95-7.09(\mathrm{~m})$ & $\begin{array}{l}6.54(\mathrm{~d}, \mathrm{~J}=7.9 \\
\mathrm{Hz})\end{array}$ & 18.62 \\
\hline & & & THF- $\mathrm{d}_{8}$ & Enol & d & $6.33(\mathrm{~d}, \mathrm{~J}=8.4 \mathrm{~Hz}) ; 7.18-7.32(\mathrm{~m})$ & $\begin{array}{l}7.69(\mathrm{~d}, \mathrm{~J}=8.0 \\
\mathrm{Hz})\end{array}$ & 18.39 \\
\hline & & & & Amide & d & $6.24(\mathrm{~d}, \mathrm{~J}=8.3 \mathrm{~Hz}) ; 7.18-7.32(\mathrm{~m}, \mathrm{ov})$ & $\begin{array}{l}8.78(\mathrm{~d}, \mathrm{~J}=8.2 \\
\mathrm{Hz})\end{array}$ & 4.78 \\
\hline & & & $\mathrm{CD}_{3} \mathrm{CN}$ & Enol & d & $6.20(\mathrm{~d}, \mathrm{~J}=7.8 \mathrm{~Hz}) ; 7.22-7.36(\mathrm{~m}, \mathrm{ov})$ & $\begin{array}{l}6.80(\mathrm{~d}, \mathrm{~J}=7.4 \\
\mathrm{Hz})\end{array}$ & 17.96 \\
\hline & & & & Amide & d & $6.09(\mathrm{~d}, \mathrm{~J}=8.1 \mathrm{~Hz}) ; 7.22-7.36(\mathrm{~m})$ & $\begin{array}{l}8.00(\mathrm{~d}, \mathrm{~J}=6.0 \\
\mathrm{Hz})\end{array}$ & 4.84 \\
\hline & & & DMF- $\mathrm{d}_{7}$ & Amide & d & $6.20(\mathrm{~d}, \mathrm{~J}=7.9 \mathrm{~Hz}) ; 7.28-7.42(\mathrm{~m})$ & $\begin{array}{l}9.50(\mathrm{~d}, \mathrm{~J}=7.4 \\
\mathrm{Hz})\end{array}$ & 5.50 \\
\hline & & & $\mathrm{DMSO}_{-} \mathrm{d}_{6}$ & Amide & d & $6.06(\mathrm{~d}, \mathrm{~J}=7.4 \mathrm{~Hz}) ; 7.26-7.38(\mathrm{~m})$ & $\begin{array}{l}9.29(\mathrm{~d}, \mathrm{~J}=7.5 \\
\mathrm{Hz})\end{array}$ & 5.18 \\
\hline
\end{tabular}

${ }^{\mathrm{a}}$ All signals are singlet, unless otherwise stated; ${ }^{\mathrm{b}} \mathrm{ov}=$ Overlaps another signal; ${ }^{\mathrm{c}}$ Not observed; ${ }^{\mathrm{d}} \mathrm{R}^{1}=\mathrm{R}^{3}, \mathrm{R}^{2}=\mathrm{H}$. 
Table S3. ${ }^{13} \mathrm{C}$ NMR Spectral Data of $\mathrm{R}^{3} \mathrm{NHCOCH}(\mathrm{CN}) \mathrm{CONR}{ }^{1} \mathrm{R}^{2} / \mathrm{R}^{3} \mathrm{NHC}(\mathrm{OH})=\mathrm{C}(\mathrm{CN}) \mathrm{CONR}{ }^{1} \mathrm{R}^{2} / \mathrm{R}^{3} \mathrm{NHCOC}(\mathrm{CN})=\mathrm{C}(\mathrm{OH}) \mathrm{NR}{ }^{1} \mathrm{R}^{2} \quad 4 / \mathbf{5} / \mathbf{6}$ in Several Solvents ${ }^{\mathrm{a}}$

\begin{tabular}{|c|c|c|c|c|c|c|c|c|c|c|}
\hline $\mathrm{R}^{1}$ & $\mathrm{R}^{2}$ & $\mathrm{R}^{3}$ & Solvent & $\begin{array}{l}\text { Enol or } \\
\text { Amide }\end{array}$ & $\begin{array}{l}\mathrm{CH} \text { or } \\
=\mathrm{C}_{\beta}^{\mathrm{b}}\end{array}$ & $\mathrm{CN}^{\mathrm{c}}$ & $\begin{array}{l}\mathrm{R}^{1} \text { and/ } \\
\text { or } \mathrm{R}^{2 \mathrm{~d}}\end{array}$ & $\mathrm{R}^{3}$ & $\mathrm{C}=\mathrm{O}$ & $=\mathrm{C}_{\alpha}$ \\
\hline \multirow[t]{6}{*}{$\mathrm{CH}_{3}$} & \multirow[t]{6}{*}{$\mathrm{CH}_{3}$} & \multirow[t]{6}{*}{$\mathrm{C}_{6} \mathrm{H}_{5}$} & \multirow[t]{2}{*}{$\mathrm{CDCl}_{3}$} & Enol & 55.60 & 120.25 & 38.33 & $\begin{array}{l}121.19(\mathrm{~d}, \mathrm{~J}=162.3 \mathrm{~Hz}) ; 124.77(\mathrm{~d} \text { of } \\
\left.\text { t, } \mathrm{J}_{\mathrm{d}}=163.0 \mathrm{~Hz}, \mathrm{~J}_{\mathrm{t}}=6.8 \mathrm{~Hz}\right), 128.89 \\
\left(\mathrm{~d} \text { of d, } \mathrm{J}_{1}=161.5 \mathrm{~Hz}, \mathrm{~J}_{2}=8.0 \mathrm{~Hz}\right) ; \\
136.59(\mathrm{t}, \mathrm{J}=8.8 \mathrm{~Hz})\end{array}$ & $171.90(\mathrm{~m})$ & $\begin{array}{l}172.06(\mathrm{~d}, \\
\mathrm{J}=3.0 \mathrm{~Hz})\end{array}$ \\
\hline & & & & Amide & 44.08 & 113.40 & $\begin{array}{l}36.71 \\
38.15\end{array}$ & $\begin{array}{l}120.25(\mathrm{~d}, \mathrm{~J}=13.8 \mathrm{~Hz}) ; 125.27(\mathrm{~d} \text { of } \\
\left.\mathrm{t}, \mathrm{J}_{\mathrm{d}}=162.3 \mathrm{~Hz}, \mathrm{~J}_{\mathrm{t}}=7.5 \mathrm{~Hz}\right) ; 128.93 \\
\left(\mathrm{~d} \text { of } \mathrm{d}, \mathrm{J}_{1}=161.5 \mathrm{~Hz}, \mathrm{~J}_{2}=8.0 \mathrm{~Hz}\right) ; \\
136.61(\mathrm{ov})\end{array}$ & $161.34(\mathrm{~s})$ & $\begin{array}{l}157.63(\mathrm{~d}, \\
\mathrm{J}=8.3 \mathrm{~Hz})\end{array}$ \\
\hline & & & \multirow[t]{2}{*}{$\mathrm{C}_{6} \mathrm{D}_{6}$} & Enol & 56.03 & 119.76 & 37.21 & $\begin{array}{l}120.98(\mathrm{~d}, \mathrm{~J}=163.0 \mathrm{~Hz}) ; 124.26(\mathrm{~d} \text { of } \\
\left.\mathrm{t}, \mathrm{J}_{\mathrm{d}}=162.3 \mathrm{~Hz}, \mathrm{~J}_{\mathrm{t}}=7.6 \mathrm{~Hz}\right) ; 128.67 \\
\left(\mathrm{~d} \text { of d, } \mathrm{J}_{1}=161.60 \mathrm{~Hz}, \mathrm{~J}_{2}=8.3 \mathrm{~Hz}\right), \\
137.26(\mathrm{t})\end{array}$ & $170.99(\mathrm{~s})$ & $172.51(\mathrm{~s})$ \\
\hline & & & & Amide & 43.95 & 113.76 & $\begin{array}{l}35.55 \\
36.80\end{array}$ & $\begin{array}{l}119.96(\mathrm{~d}, \mathrm{~J}=163.0 \mathrm{~Hz}) ; 124.76(\mathrm{~d} \text { of } \\
\left.\mathrm{t}, \mathrm{J}_{\mathrm{d}}=161.6 \mathrm{~Hz}, \mathrm{~J}_{\mathrm{t}}=7.6 \mathrm{~Hz}\right) ; 128.81 \\
(\mathrm{ov}) ; 137.48(\mathrm{ov})\end{array}$ & $161.25(\mathrm{~s})$ & $157.82(\mathrm{~s})$ \\
\hline & & & \multirow[t]{2}{*}{ THF- $\mathrm{d}_{8}$} & Enol & 56.61 & 119.44 & 38.51 & $\begin{array}{l}120.73(\mathrm{~d}, \mathrm{~J}=159.2 \mathrm{~Hz}) ; 125.09(\mathrm{~d} \text { of } \\
\left.\text { t, } \mathrm{J}_{\mathrm{d}}=162.3 \mathrm{~Hz}, \mathrm{~J}_{\mathrm{t}}=6.8 \mathrm{~Hz}\right) ; 129.32 \\
\left(\mathrm{~d} \text { of d, } \mathrm{J}_{1}=161.5 \mathrm{~Hz}, \mathrm{~J}_{2}=8.3 \mathrm{~Hz}\right) ; \\
138.64(\mathrm{t}, \mathrm{J}=9.8 \mathrm{~Hz})\end{array}$ & $172.80(\mathrm{~m})$ & $\begin{array}{l}173.74(\mathrm{~d}, \\
\mathrm{J}=3.0 \mathrm{~Hz})\end{array}$ \\
\hline & & & & Amide & 45.75 & 114.52 & $\begin{array}{l}36.35 \\
37.96\end{array}$ & $\begin{array}{l}122.51(\mathrm{~d}, \mathrm{~J}=163.0 \mathrm{~Hz}) ; 125.11(\mathrm{ov}) ; \\
129.47\left(\mathrm{~d} \text { of d, } \mathrm{J}_{1}=161.3 \mathrm{~Hz}, \mathrm{~J}_{2}=8.2\right.\end{array}$ & $162.11(\mathrm{~m})$ & $\begin{array}{l}159.67(\mathrm{~d} \\
\mathrm{J}=8.3 \mathrm{~Hz})\end{array}$ \\
\hline
\end{tabular}




\begin{tabular}{|c|c|c|c|c|c|c|c|}
\hline \multirow{3}{*}{$\left(\mathrm{CD}_{3}\right)_{2} \mathrm{CO}$} & & & & & $\mathrm{Hz}) ; 139.31(\mathrm{t}, \mathrm{J}=9.7 \mathrm{~Hz})$ & & \\
\hline & Enol & 55.46 & 118.69 & 37.71 & $\begin{array}{l}119.67(\mathrm{~d}, \mathrm{ov}) ; 121.76(\mathrm{~d}, \mathrm{~J}=161.6 \\
\mathrm{Hz}) ; 128.57(\mathrm{~d}, \mathrm{~J}=160.3 \mathrm{~Hz}) ; 137.42 \\
(\mathrm{t})\end{array}$ & $171.49(\mathrm{~s})$ & $172.75(\mathrm{~s})$ \\
\hline & Amide & 45.14 & 113.80 & $\begin{array}{l}35.44 \\
37.02\end{array}$ & $\begin{array}{l}119.78(\mathrm{~d}, \mathrm{~J}=163.0 \mathrm{~Hz}) ; 124.45(\mathrm{~d}, \mathrm{~J} \\
=160.8 \mathrm{~Hz}) ; 128.79(\mathrm{~d}, \mathrm{~J}=161.5 \\
\mathrm{Hz}) ; 138.08(\mathrm{t}, \mathrm{J}=8.1 \mathrm{~Hz})\end{array}$ & $161.06(\mathrm{~s})$ & $\begin{array}{l}159.09(\mathrm{~s}) \\
(\mathrm{d}, \mathrm{J}=9.3 \\
\mathrm{Hz})\end{array}$ \\
\hline \multirow[t]{2}{*}{$\mathrm{CD}_{3} \mathrm{CN}$} & Enol & 55.41 & e & 37.90 & $\begin{array}{l}122.38(\mathrm{~d}, \text { ov }) ; 124.92(\mathrm{~d}, \text { ov }) ; 128.72 \\
\left.\left(\mathrm{~d} \text { of d, } \mathrm{J}_{1}=11.5 \mathrm{~Hz}\right), \mathrm{J}_{2}=8.3 \mathrm{~Hz}\right) \\
136.96(\mathrm{t}, \text { weak })\end{array}$ & e & \\
\hline & Amide & 45.06 & 113.93 & $\begin{array}{l}35.66 \\
37.29\end{array}$ & $\begin{array}{l}120.15(\mathrm{~d}, \mathrm{~J}=163.8 \mathrm{~Hz}) ; 124.93(\mathrm{~d} \text { of } \\
\left.\mathrm{t}, \mathrm{J}_{\mathrm{d}}=163.0 \mathrm{~Hz}, \mathrm{~J}_{\mathrm{t}}=7.5 \mathrm{~Hz}\right) ; 128.98 \\
\left(\mathrm{~d} \text { of d, } \mathrm{J}_{1}=162.3 \mathrm{~Hz}, \mathrm{~J}_{2}=8.3 \mathrm{~Hz}\right) ; \\
137.58(\mathrm{t}, \mathrm{J}=9.8 \mathrm{~Hz})\end{array}$ & $161.06(\mathrm{~m})$ & $\begin{array}{l}159.68(\mathrm{~d}, \\
\mathrm{J}=5.3 \mathrm{~Hz})\end{array}$ \\
\hline DMF-d 7 & Amide & 45.73 & 114.39 & $\begin{array}{l}35.31 \\
36.91\end{array}$ & $\begin{array}{l}119.45(\mathrm{~d}, \mathrm{~J}=163.0 \mathrm{~Hz}) ; 124.16(\mathrm{~d} \text { of } \\
\left.\mathrm{t}, \mathrm{J}_{\mathrm{d}}=163.0 \mathrm{~Hz}, \mathrm{~J}_{\mathrm{t}}=7.5 \mathrm{~Hz}\right) ; 128.85 \\
\left(\mathrm{~d} \text { of d, } \mathrm{J}_{1}=160.0 \mathrm{~Hz}, \mathrm{~J}_{2}=8.3 \mathrm{~Hz}\right) ; \\
138.68(\mathrm{t}, \mathrm{J}=9.9 \mathrm{~Hz})\end{array}$ & $161.23(\mathrm{~m})$ & $\begin{array}{l}159.68(\mathrm{~d}, \\
\mathrm{J}=5.3 \mathrm{~Hz})\end{array}$ \\
\hline $\mathrm{DMSO}_{-} \mathrm{d}_{6}$ & Amide & 45.61 & 114.46 & $\begin{array}{l}35.69 \\
37.13\end{array}$ & $\begin{array}{l}119.39(\mathrm{~d}, \mathrm{~J}=163.8 \mathrm{~Hz}) ; 124.17(\mathrm{~d} \text { of } \\
\left.\mathrm{t}, \mathrm{J}_{\mathrm{d}}=163.8 \mathrm{~Hz}, \mathrm{~J}_{\mathrm{t}}=7.5 \mathrm{~Hz}\right) ; 128.87 \\
\left(\mathrm{~d} \text { of d, } \mathrm{J}_{1}=163.0 \mathrm{~Hz} ; \mathrm{J}_{2}=8.3 \mathrm{~Hz}\right) ; \\
138.04(\mathrm{t}, \mathrm{J}=9.1 \mathrm{~Hz})\end{array}$ & $\begin{array}{l}160.90(\mathrm{~m}, \\
\mathrm{J}=3.8 \mathrm{~Hz})\end{array}$ & $\begin{array}{l}159.27(\mathrm{~d} \\
\text { of } \mathrm{d}, \mathrm{J}_{1}= \\
6.8 \mathrm{~Hz}, \mathrm{~J}_{2} \\
=2.3 \mathrm{~Hz})\end{array}$ \\
\hline \multirow[t]{2}{*}{$\mathrm{CDCl}_{3}$} & Enol & 55.08 & 120.35 & 38.17 & $\begin{array}{l}55.26(\mathrm{q}, \mathrm{J}=143.7 \mathrm{~Hz}) ; 113.96(\mathrm{~d}, \mathrm{~J}= \\
160.2 \mathrm{~Hz}) ; 123.47(\mathrm{~d}, \mathrm{~J}=162.3 \mathrm{~Hz}) ; \\
129.19(\mathrm{t}, \mathrm{J}=8.9 \mathrm{~Hz}) ; 156.88(\mathrm{~m})\end{array}$ & $171.02(\mathrm{~m})$ & $\begin{array}{l}172.03(\mathrm{~d}, \\
\mathrm{J}=3.4 \mathrm{~Hz})\end{array}$ \\
\hline & Amide & 44.19 & 113.56 & $\begin{array}{l}36.59 \\
38.04(\mathrm{q}, \\
\mathrm{J}=143.2 \\
\mathrm{~Hz})\end{array}$ & $\begin{array}{l}55.26(\mathrm{q}, \mathrm{ov}) ; 113.92(\mathrm{~d}, \mathrm{~J}=160.1 \\
\mathrm{Hz}) ; 121.95(\mathrm{~d}, \mathrm{~J}=161.5 \mathrm{~Hz}) ; 129.69 \\
(\mathrm{t}, \mathrm{J}=9.0 \mathrm{~Hz}), 156.88(\mathrm{ov})\end{array}$ & $161.28(\mathrm{~s})$ & $\begin{array}{l}157.58(\mathrm{~d}, \\
\mathrm{J}=6.6 \mathrm{~Hz})\end{array}$ \\
\hline \multirow[t]{2}{*}{$\mathrm{C}_{6} \mathrm{D}_{6}$} & Enol & 55.63 & 119.97 & 37.20 & $\begin{array}{l}54.55(\mathrm{q}, \mathrm{J}=143.5 \mathrm{~Hz}) ; 113.90(\mathrm{~d}, \mathrm{~J}= \\
159.3 \mathrm{~Hz}) ; 123.15(\mathrm{~d}, \mathrm{~J}=161.2 \mathrm{~Hz}) ; \\
130.01(\mathrm{t}) ; 156.93(\mathrm{~s})\end{array}$ & $171.13(\mathrm{~s})$ & $172.53(\mathrm{~s})$ \\
\hline & Amide & 44.03 & 114.00 & 35.57 & $54.55(\mathrm{q}, \mathrm{ov}) ; 113.90(\mathrm{ov}) ; 121.67(\mathrm{~d}$ & $161.38(\mathrm{~s})$ & $157.67(d)$ \\
\hline
\end{tabular}




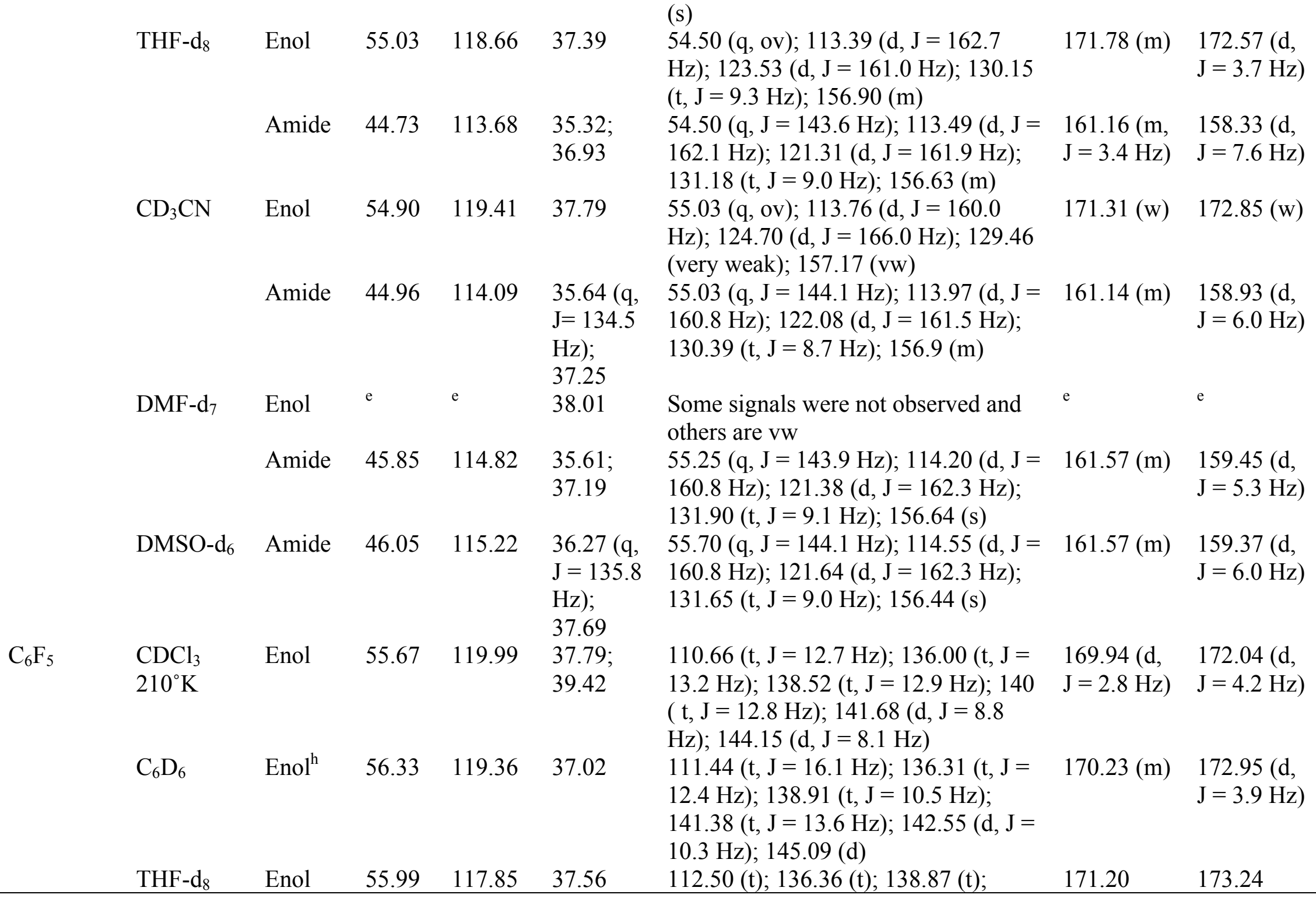




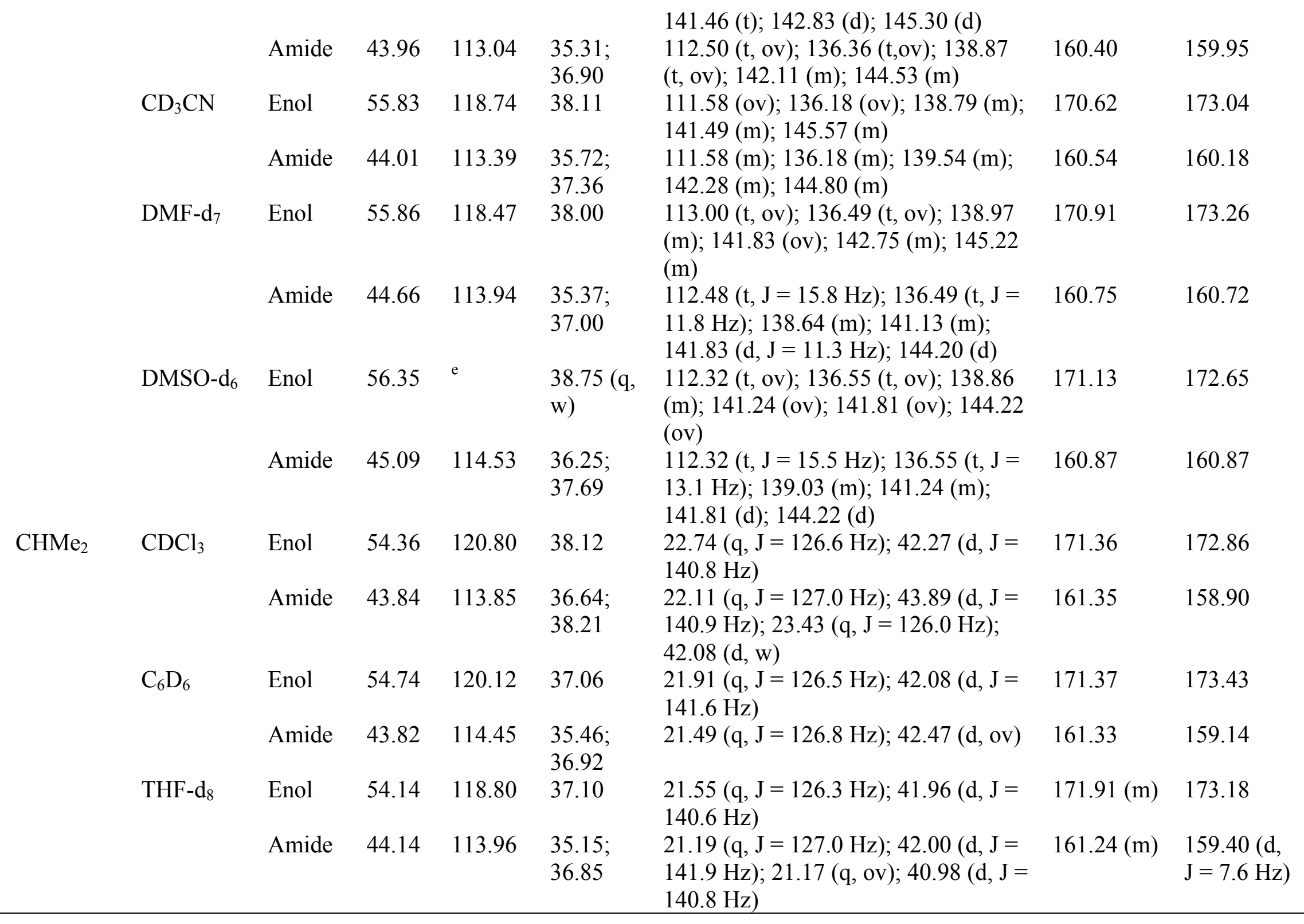




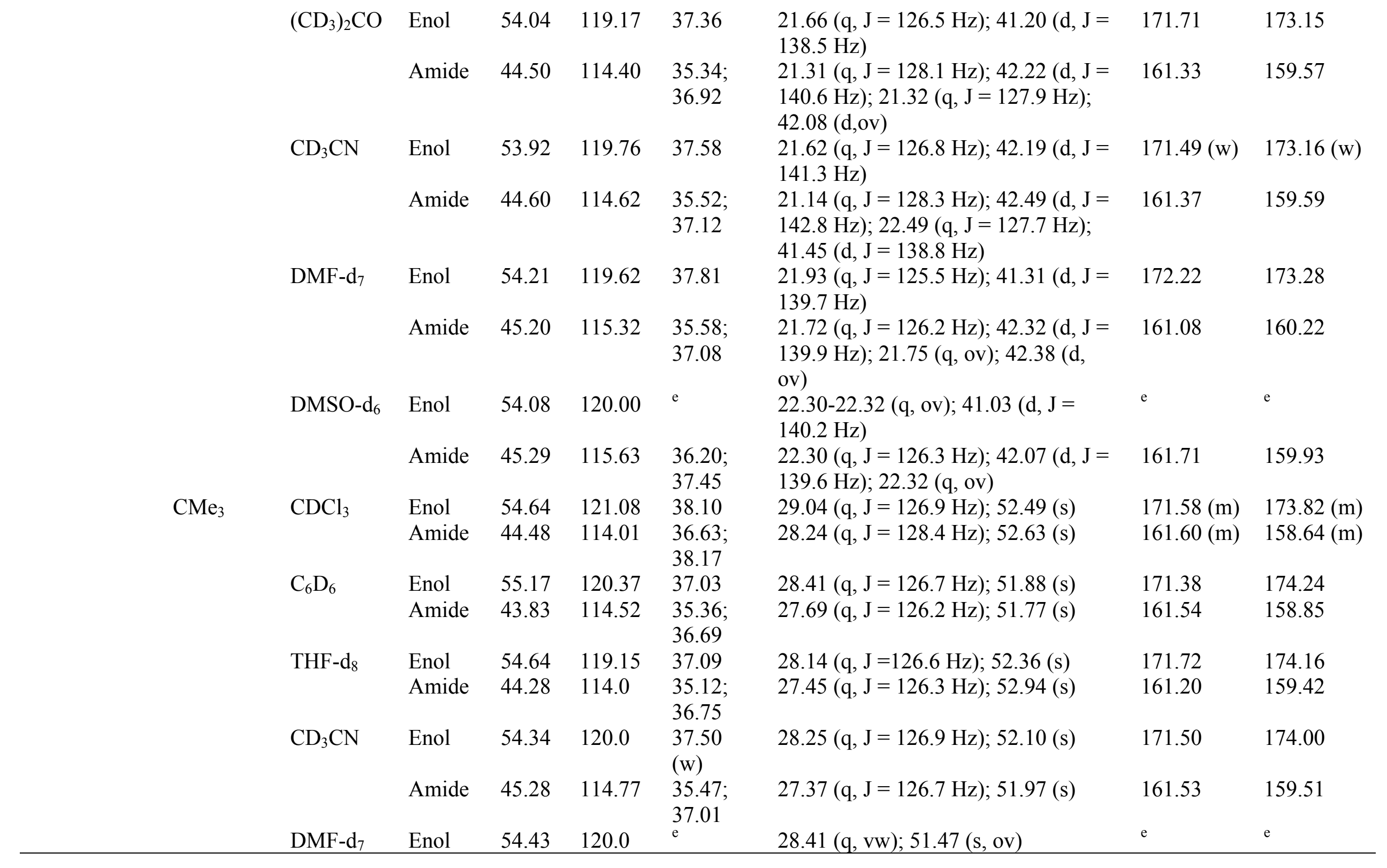




\begin{tabular}{|c|c|c|c|c|c|c|c|c|c|c|}
\hline & & & & & & & & & & \\
\hline & & & & & $(\mathrm{w})$ & (w) & & & & \\
\hline & & & & Amide & $\begin{array}{l}45.74 \\
(\mathrm{~d} . \mathrm{J}=\end{array}$ & $\begin{array}{l}115.2 \\
(\mathrm{~d} J=\end{array}$ & $\begin{array}{l}35.32 \\
3675\end{array}$ & $27.70(\mathrm{q}, \mathrm{J}=126.2 \mathrm{~Hz}) ; 51.47(\mathrm{~s})$ & $161.71(\mathrm{~m})$ & $160.06(\mathrm{~d}$ \\
\hline & & & & & 135.9 & 11.1 & & & & $6.7 \mathrm{~Hz}, \mathrm{~J}_{2}$ \\
\hline & & & & & $\mathrm{Hz})$ & $\mathrm{Hz})$ & & & & $=2.7 \mathrm{~Hz})$ \\
\hline & & & $\mathrm{DMSO}_{-} \mathrm{d}_{6}$ & Amide & 45.48 & 115.28 & 35.57 & $27.87(\mathrm{q}, \mathrm{J}=126.7 \mathrm{~Hz}) ; 51.14(\mathrm{~s})$ & $161.24(\mathrm{~m})$ & $159.40(\mathrm{~d}$, \\
\hline & & & & & $(\mathrm{d}, \mathrm{J}=$ & $(\mathrm{d}, \mathrm{J}=$ & 36.73 & & & $\mathrm{~J}=6.8 \mathrm{~Hz})$ \\
\hline & & & & & 136.4 & 10.6 & & & & \\
\hline & & & & & $\mathrm{Hz})$ & $\mathrm{Hz})$ & & & & \\
\hline & & $\mathrm{CPh}_{3}$ & $\mathrm{CDCl}_{3}$ & Enol & 55.59 & 120.87 & 38.13 & $127.13(\mathrm{~d}, \mathrm{~J}=160.9 \mathrm{~Hz}) ; 128.06(\mathrm{~d}, \mathrm{~J}$ & $171.15(\mathrm{~m})$ & $173.50(\mathrm{~m})$ \\
\hline & & & & & & & & $=160.2 \mathrm{~Hz}) ; 128.41(\mathrm{~d}, \mathrm{~J}=158.2$ & & \\
\hline & & & & Amide & 44.72 & 113.54 & 36.72 ; & $127.30(\mathrm{~d}, \mathrm{~J}=160.9 \mathrm{~Hz}) ; 128.11(\mathrm{~d}, \mathrm{~J}$ & $161.31(\mathrm{~m})$ & $158.37(\mathrm{~m})$ \\
\hline & & & & & & & 38.11 & $\begin{array}{l}=160.2 \mathrm{~Hz}) ; 128.30(\mathrm{~d}, \mathrm{~J}=158.2 \\
\mathrm{Hz}) ; 143.54(\mathrm{t}, \mathrm{J}=7.6 \mathrm{~Hz})\end{array}$ & & \\
\hline & & & $\mathrm{CD}_{3} \mathrm{CN}$ & Enol & 55.36 & 120.52 & e & $126.99 ; 127.81 ; 128.32 ; 143.54(\mathrm{t}, \mathrm{ov})$ & e & e \\
\hline & & & & Amide & 44.80 & 114.29 & $\begin{array}{l}35.60 \\
37.19\end{array}$ & $\begin{array}{l}127.17(\mathrm{~d}, \mathrm{~J}=161.9 \mathrm{~Hz}) ; 127.91(\mathrm{~d}, \mathrm{~J} \\
=160.8 \mathrm{~Hz}) ; 128.48(\mathrm{~d}, \mathrm{~J}=159.4 \\
\mathrm{Hz}) ; 143.94(\mathrm{t}, \mathrm{J}=7.5 \mathrm{~Hz})\end{array}$ & $161.65(\mathrm{~m})$ & $159.57(\mathrm{~m})$ \\
\hline & & & $\mathrm{DMSO}^{-\mathrm{d}_{6}}$ & Amide & 45.90 & 115.43 & 36.07; & $127.21(\mathrm{~d}, \mathrm{~J}=162.1 \mathrm{~Hz}) ; 128.15(\mathrm{~d}, \mathrm{~J}$ & $161.87(\mathrm{~m})$ & $160.40(\mathrm{~m})$ \\
\hline & & & & & & & 37.29 & $\begin{array}{l}=161.6 \mathrm{~Hz}), 128.84(\mathrm{~d}, \mathrm{~J}=160.3 \\
\mathrm{Hz}) ; 144.30(\mathrm{t}, \mathrm{J}=7.2 \mathrm{~Hz})\end{array}$ & & \\
\hline $\mathrm{CH}_{3}$ & $\mathrm{H}$ & $\mathrm{C}_{6} \mathrm{H}_{5}$ & $\mathrm{CDCl}_{3}$ & Enol & 55.80 & 118.37 & 26.76 & $121.44(\mathrm{~d}, \mathrm{~J}=162.3 \mathrm{~Hz}) ; 125.12(\mathrm{~d}$ of & $170.89(\mathrm{~d}$ & $171.19(\mathrm{~m}$, \\
\hline & & & & & & & & $\begin{array}{l}\left.\mathrm{t}, \mathrm{J}_{\mathrm{d}}=163.0 \mathrm{~Hz}, \mathrm{~J}_{\mathrm{t}}=6.8 \mathrm{~Hz}\right) ; 129.11 \\
\left.\left(\mathrm{~d} \text { of d, } \mathrm{J}_{1}=160.8 \mathrm{~Hz}\right), \mathrm{J}_{2}=7.6 \mathrm{~Hz}\right) \\
136.36(\mathrm{t}, \mathrm{J}=9.1 \mathrm{~Hz})\end{array}$ & $\mathrm{J}=3.8 \mathrm{~Hz})$ & $\mathrm{J}=4.5 \mathrm{~Hz})$ \\
\hline & & & & Amide & 47.15 & 113.89 & 27.34 & $120.35(\mathrm{~d}, \mathrm{~J}=163.8 \mathrm{~Hz}) ; 125.54(\mathrm{~d}$ & $161.56(\mathrm{~s})$ & $158.02(\mathrm{~s})$ \\
\hline & & & & & & & & $\begin{array}{l}\left.\text { of t }, J_{d}=163.0 \mathrm{~Hz}, J_{t}=7.6 \mathrm{~Hz}\right) \\
129.11(\mathrm{ov}) ; 136.62(\mathrm{t}, \mathrm{w})\end{array}$ & & \\
\hline & & & $\mathrm{C}_{6} \mathrm{D}_{6}$ & Enol & 56.03 & 117.62 & 25.78 & $120.95(\mathrm{~d}, \mathrm{~J}=162.6 \mathrm{~Hz}) ; 124.44(\mathrm{~d}$ of & $171.05(\mathrm{~s})$ & $173.09(\mathrm{~s})$ \\
\hline & & & & & & & & $\left.\mathrm{t}, \mathrm{J}_{\mathrm{d}}=162.3 \mathrm{~Hz}, \mathrm{~J}_{\mathrm{t}}=7.3 \mathrm{~Hz}\right) ; 128.66$ & & \\
\hline & & & & Amide & 46.85 & e & 26.07 & $120.95(\mathrm{~d}, \mathrm{~J}=162.6 \mathrm{~Hz}) ; 124.44(\mathrm{~d}$ of & $172.06(\mathrm{~s})$ & $173.09(\mathrm{~s})$ \\
\hline
\end{tabular}




\begin{tabular}{|c|c|c|c|c|c|c|c|}
\hline \multirow{3}{*}{ THF- $\mathrm{d}_{8}$} & \multirow[b]{2}{*}{ Enol } & \multirow[b]{2}{*}{55.49} & \multirow[b]{2}{*}{116.31} & \multirow[b]{2}{*}{25.73} & \multicolumn{2}{|l|}{$\left(\mathrm{U} 01 \mathrm{u}, \mathrm{J}_{2}-0.0 \mathrm{HZ}\right), 150.0 /(\mathrm{l})$} & \multirow[b]{2}{*}{$173.36(\mathrm{~s})$} \\
\hline & & & & & $\begin{array}{l}121.55(\mathrm{~d}, \mathrm{~J}=162.6 \mathrm{~Hz}) ; 123.94(\mathrm{~d}, \mathrm{~J} \\
=161.4 \mathrm{~Hz}) ; 128.23(\mathrm{~d}, \mathrm{~J}=158.2 \\
\mathrm{Hz}) ; 137.56(\mathrm{t}, \mathrm{J}=10.0 \mathrm{~Hz})\end{array}$ & $\begin{array}{l}171.43(\mathrm{~d} \\
\mathrm{J}=3.3 \mathrm{~Hz})\end{array}$ & \\
\hline & Amide & 46.96 & 113.84 & 26.08 & $\begin{array}{l}119.45(\mathrm{~d}, \mathrm{~J}=162.0 \mathrm{~Hz}) ; 124.05(\mathrm{~d}, \\
\text { ov }) ; 128.47(\mathrm{~d}, \mathrm{~J}=160.9 \mathrm{~Hz}) ; 138.22 \\
(\mathrm{t}, \mathrm{w})\end{array}$ & $161.20(\mathrm{~s})$ & $158.86(\mathrm{~s})$ \\
\hline \multirow[t]{2}{*}{$\mathrm{CD}_{3} \mathrm{CN}$} & Enol & 55.50 & 116.83 & 25.89 & $\begin{array}{l}122.43(\mathrm{~d}, \mathrm{~J}=163.3 \mathrm{~Hz}) ; 124.88(\mathrm{~d}, \mathrm{~J} \\
=163.8 \mathrm{~Hz}) ; 128.70\left(\mathrm{~d} \text { of } \mathrm{d}, \mathrm{J}_{1}=\right. \\
\left.162.3 \mathrm{~Hz}), \mathrm{J}_{2}=8.2 \mathrm{~Hz}\right) ; 136.76(\mathrm{t})\end{array}$ & $171.49(\mathrm{~s})$ & $173.30(\mathrm{~s})$ \\
\hline & Amide & 47.10 & 114.28 & 26.33 & $\begin{array}{l}120.10(\mathrm{~d}, \mathrm{~J}=164.2 \mathrm{~Hz}) ; 124.95(\mathrm{~d}, \mathrm{~J} \\
=163.6 \mathrm{~Hz}) ; 128.98\left(\mathrm{~d} \text { of } \mathrm{d}, \mathrm{J}_{1}=\right. \\
\left.163.7 \mathrm{~Hz}, \mathrm{~J}_{2}=7.9 \mathrm{~Hz}\right) ; 137.50(\mathrm{t})\end{array}$ & $161.18(\mathrm{~s})$ & $159.16(\mathrm{~s})$ \\
\hline \multirow[t]{2}{*}{$\mathrm{DMF}-\mathrm{d}_{8}$} & Enol & 55.46 & 116.76 & 26.12 & $\begin{array}{l}122.06(\mathrm{~d}, \mathrm{~J}=162.2 \mathrm{~Hz}) ; 124.14(\mathrm{ov}) \\
128.41(\mathrm{~d}, \mathrm{~J}=160.6 \mathrm{~Hz}) ; 137.68(\mathrm{t})\end{array}$ & $171.45(\mathrm{~s})$ & $173.09(\mathrm{~s})$ \\
\hline & Amide & 47.22 & 114.76 & 26.18 & $\begin{array}{l}119.37(\mathrm{~d}, \mathrm{~J}=162.7 \mathrm{~Hz}) ; 124.14(\mathrm{~d}, \mathrm{~J} \\
=158.8 \mathrm{~Hz}) ; 128.75(\mathrm{~d}, \mathrm{~J}=158.4 \\
\mathrm{Hz}) ; 138.48(\mathrm{t}, \mathrm{J}=9.1 \mathrm{~Hz})\end{array}$ & $\begin{array}{l}161.14(\mathrm{~m}, \\
\mathrm{J}=3.5 \mathrm{~Hz})\end{array}$ & $\begin{array}{l}159.57(\mathrm{~d} \\
\mathrm{J}=5.0 \mathrm{~Hz})\end{array}$ \\
\hline \multirow[t]{2}{*}{$\mathrm{DMSO}^{-\mathrm{d}_{6}}$} & Enol & 55.80 & 117.90 & e & $\begin{array}{l}122.84(\mathrm{~d}, \mathrm{~J}=165.9 \mathrm{~Hz}) ; 124.74(\mathrm{ov}) \\
128.97(\mathrm{~d}, \mathrm{~J}=163.8 \mathrm{~Hz}) ; 137.81(\mathrm{t}, \\
\mathrm{w})\end{array}$ & $171.49(\mathrm{~s})$ & $172.99(\mathrm{~s})$ \\
\hline & Amide & 47.55 & 115.50 & 26.99 & $\begin{array}{l}119.93(\mathrm{~d}, \mathrm{~J}=162.6 \mathrm{~Hz}) ; 124.74(\mathrm{~d}, \mathrm{~J} \\
=159.7 \mathrm{~Hz}) ; 129.41\left(\mathrm{~d} \text { of } \mathrm{d}, \mathrm{J}_{1}=\right. \\
\left.158.1 \mathrm{~Hz}, \mathrm{~J}_{2}=7.6 \mathrm{~Hz}\right) ; 138.60(\mathrm{t}, \mathrm{J}= \\
8.9 \mathrm{~Hz})\end{array}$ & $161.30(\mathrm{~s})$ & $\begin{array}{l}159.78(\mathrm{~d} \\
\mathrm{J}=5.1 \mathrm{~Hz})\end{array}$ \\
\hline \multirow[t]{2}{*}{$\mathrm{CDCl}_{3}$} & Enol & 55.40 & 118.25 & 26.70 & $\begin{array}{l}55.45(\mathrm{q}, \mathrm{J}=143.8 \mathrm{~Hz}) ; 114.26(\mathrm{~d} \text { of } \\
\left.\mathrm{d}, \mathrm{J}_{1}=160.2 \mathrm{~Hz}, \mathrm{~J}_{2}=5.2 \mathrm{~Hz}\right) ; 123.67 \\
(\mathrm{~d}, \mathrm{~J}=161.7 \mathrm{~Hz}) ; 128.74(\mathrm{t}) ; 157.27 \\
(\mathrm{~s})\end{array}$ & $\begin{array}{l}170.82(\mathrm{~d}, \\
\mathrm{J}=3.6 \mathrm{~Hz})\end{array}$ & $173.04(\mathrm{~s})$ \\
\hline & Amide & 46.93 & e & 27.28 & $\begin{array}{l}55.45(\mathrm{ov}) ; 114.52\left(\mathrm{~d} \text { of } \mathrm{d}, \mathrm{J}_{1}=160\right. \\
\left.\mathrm{Hz}, \mathrm{J}_{2}=5.5 \mathrm{~Hz}\right) ; 122.0(\mathrm{~d}, \mathrm{ov})\end{array}$ & e & e \\
\hline $\mathrm{C}_{6} \mathrm{D}_{6}$ & Enol & 55.72 & 117.69 & 25.76 & $54.52 ; 113.88 ; 123.14 ; 129.24 ; 157.10$ & 171.06 & 173.08 \\
\hline
\end{tabular}




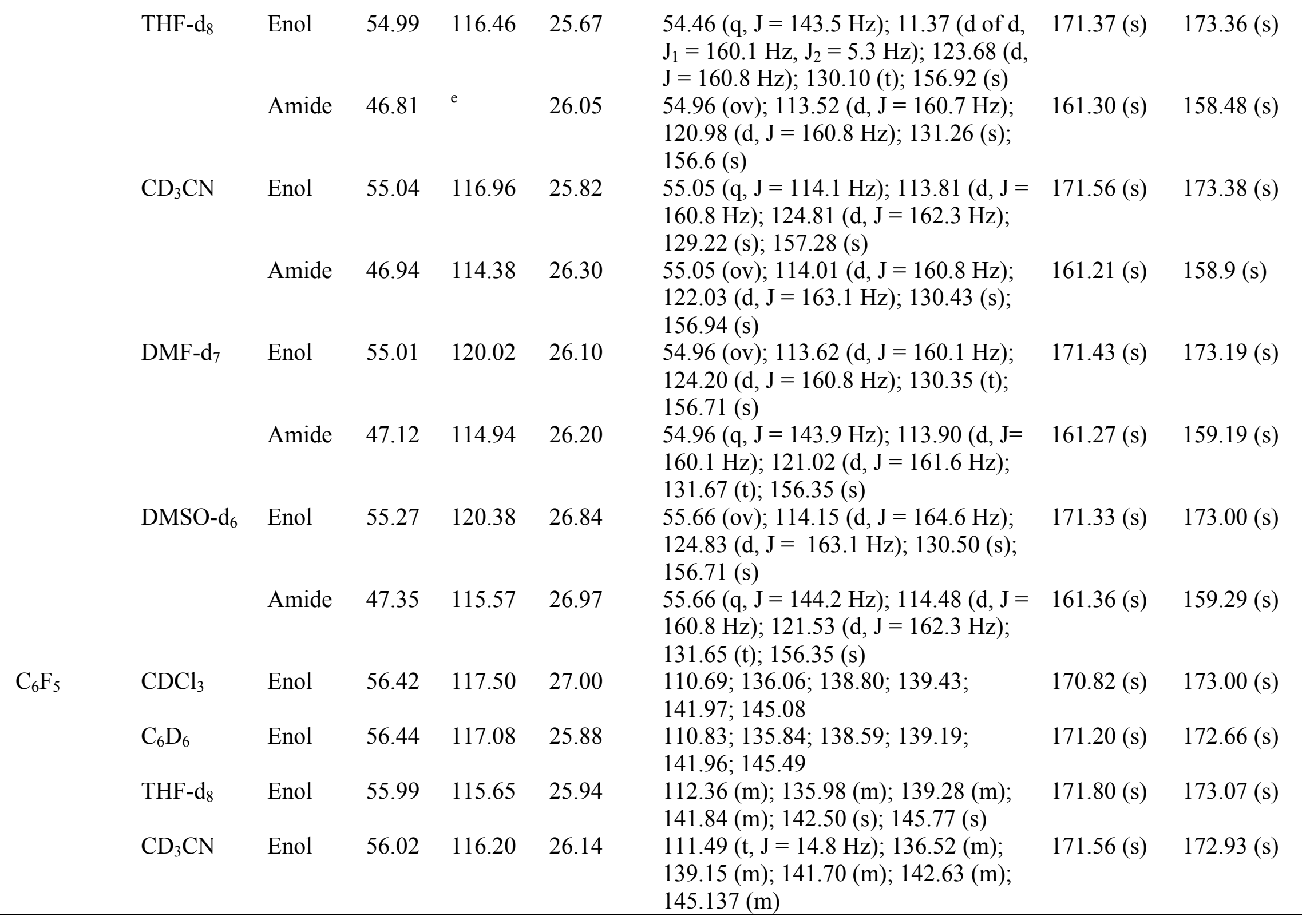



(m, ov); 141.70 (m, ov); 142.63 (m, ov); 145.137 (m, ov)

\begin{tabular}{|c|c|c|c|c|c|c|c|c|}
\hline & DMF-d 7 & Enol & 55.92 & 116.33 & 26.40 & $\begin{array}{l}112.70(\mathrm{~m}) ; 136.38(\mathrm{~m}) ; 138.87(\mathrm{~m}) \\
141.23(\mathrm{~m}) ; 142.72(\mathrm{~m}) ; 145.20(\mathrm{~m})\end{array}$ & $171.79(\mathrm{~s})$ & $172.61(\mathrm{~s})$ \\
\hline & & Amide & 46.18 & 114.38 & 26.2 & $\begin{array}{l}192.70(\mathrm{ov}) ; 136.38(\mathrm{ov}) ; 138.75(\mathrm{~m}) \\
141.69(\mathrm{~m}) ; 142.72(\mathrm{ov}) ; 144.25(\mathrm{~m})\end{array}$ & $162.21(\mathrm{~s})$ & $160.67(\mathrm{~s})$ \\
\hline & $\mathrm{DMSO}_{-} \mathrm{d}_{6}$ & Enol & 56.11 & 117.56 & 27.20 & $\begin{array}{l}112.76(\mathrm{~m}) ; 136.06(\mathrm{~m}) ; 138.32(\mathrm{~m}) \\
139.37(\mathrm{~m}) ; 141.64(\mathrm{~m}) ; 145.49(\mathrm{~m})\end{array}$ & $171.55(\mathrm{~s})$ & $172.36(\mathrm{~s})$ \\
\hline & & Amide & 46.43 & 115.11 & 27.20 & $\begin{array}{l}112.76(\mathrm{ov}) ; 136.06(\mathrm{ov}) ; 138.50(\mathrm{~m}) \\
139.37(\mathrm{ov}) ; 142.19(\mathrm{~m}) ; 144.71(\mathrm{~m})\end{array}$ & $160.87(\mathrm{~s})$ & $160.67(\mathrm{~s})$ \\
\hline \multirow[t]{11}{*}{$\mathrm{CHMe}_{2}$} & $\mathrm{CDCl}_{3}$ & Enol & 54.39 & 118.80 & 26.40 & $\begin{array}{l}22.78(\mathrm{q}, \mathrm{J}=126.6 \mathrm{~Hz}) ; 42.47(\mathrm{~d}, \mathrm{~J}= \\
141.9 \mathrm{~Hz})\end{array}$ & $171.63(\mathrm{~s})$ & $172.95(\mathrm{~s})$ \\
\hline & & Amide & 46.45 & 114.13 & 27.08 & $\begin{array}{l}22.11(\mathrm{q}, \mathrm{J}=124.1 \mathrm{~Hz}) ; 43.02(\mathrm{~d}, \mathrm{~J}= \\
140.8 \mathrm{~Hz}) ; 22.13(\mathrm{q}, \mathrm{ov})\end{array}$ & $161.12(\mathrm{~s})$ & $159.50(\mathrm{~s})$ \\
\hline & $\mathrm{C}_{6} \mathrm{D}_{6}$ & Enol & 54.63 & 118.40 & 25.66 & $\begin{array}{l}21.82(\mathrm{q}, \mathrm{J}=126.5 \mathrm{~Hz}) ; 42.07(\mathrm{~d}, \mathrm{~J}= \\
141.5 \mathrm{~Hz})\end{array}$ & $172.13(\mathrm{~s})$ & $173.22(\mathrm{~s})$ \\
\hline & THF- $\mathrm{d}_{8}$ & Enol & 54.00 & 116.83 & 25.39 & $\begin{array}{l}21.65(\mathrm{q}, \mathrm{J}=126.3 \mathrm{~Hz}) ; 41.80(\mathrm{~d}, \mathrm{~J}= \\
140.9 \mathrm{~Hz})\end{array}$ & $171.96(\mathrm{~s})$ & $173.30(\mathrm{~s})$ \\
\hline & & Amide & 45.96 & 114.14 & 25.47 & $21.22(\mathrm{q}, \mathrm{J}=124.3 \mathrm{~Hz} ; 42.00(\mathrm{~d}, \mathrm{ov})$ & $161.16(\mathrm{~s})$ & $159.85(\mathrm{~s})$ \\
\hline & $\mathrm{CD}_{3} \mathrm{CN}$ & Enol & 53.88 & 117.51 & 25.60 & $\begin{array}{l}21.62(\mathrm{q}, \mathrm{J}=126.7 \mathrm{~Hz}) ; 42.23(\mathrm{~d}, \mathrm{~J}= \\
141.1 \mathrm{~Hz})\end{array}$ & $171.87(\mathrm{~s})$ & $173.24(\mathrm{~s})$ \\
\hline & & Amide & 46.29 & 114.67 & 26.21 & $\begin{array}{l}21.16(q, J=126.7 \mathrm{~Hz}) ; 42.52(\mathrm{~d}, \mathrm{~J}= \\
141.1 \mathrm{~Hz})\end{array}$ & $161.35(\mathrm{~s})$ & $159.93(\mathrm{~s})$ \\
\hline & DMF-d 7 & Enol & 53.78 & 117.38 & 25.87 & $\begin{array}{l}21.69(\mathrm{q}, \mathrm{J}=126.3 \mathrm{~Hz}) ; 41.89(\mathrm{~d}, \mathrm{~J}= \\
140.5 \mathrm{~Hz})\end{array}$ & $171.71(\mathrm{~s})$ & $173.22(\mathrm{~s})$ \\
\hline & & Amide & 46.17 & 115.14 & 26.13 & $\begin{array}{l}21.39(\mathrm{q}, \mathrm{J}=126.4 \mathrm{~Hz}) ; 42.00(\mathrm{~d}, \mathrm{~J}= \\
140.5 \mathrm{~Hz}) ; 21.46(\mathrm{q}, \mathrm{ov})\end{array}$ & $161.46(\mathrm{~s})$ & $160.11(\mathrm{~s})$ \\
\hline & $\mathrm{DMSO}_{-} \mathrm{d}_{6}$ & Enol & 53.88 & 118.36 & 26.77 & $22.55(\mathrm{q}, \mathrm{J}=126.3 \mathrm{~Hz}) ; 42.03(\mathrm{~d}, \mathrm{ov})$ & $171.59(\mathrm{~s})$ & $173.13(\mathrm{~s})$ \\
\hline & & Amide & 46.41 & 115.75 & 26.91 & $\begin{array}{l}22.32(\mathrm{q}, \mathrm{J}=126.3 \mathrm{~Hz}) ; 42.18(\mathrm{~d}, \mathrm{~J}= \\
140.2 \mathrm{~Hz}) ; 22.39(\mathrm{q}, \mathrm{J}=126.3 \mathrm{~Hz})\end{array}$ & $161.55(\mathrm{~m})$ & $160.12(\mathrm{~m})$ \\
\hline \multirow[t]{2}{*}{$\mathrm{CMe}_{3}$} & $\mathrm{CDCl}_{3}$ & Enol & 54.79 & 118.95 & 26.39 & $29.23(\mathrm{q}, \mathrm{J}=126.6 \mathrm{~Hz}) ; 52.95(\mathrm{~s})$ & $172.69(\mathrm{~s})$ & $173.07(\mathrm{~s})$ \\
\hline & & Amide & 47.17 & 116.12 & 27.13 & $28.30(\mathrm{q}, \mathrm{J}=125.7 \mathrm{~Hz}) ; 52.79(\mathrm{~s})$ & $161.55(\mathrm{~s})$ & $159.20(\mathrm{~s})$ \\
\hline
\end{tabular}




\begin{tabular}{|c|c|c|c|c|c|c|c|c|c|c|}
\hline & & & $\mathrm{C}_{6} \mathrm{D}_{6}$ & $\begin{array}{l}\text { Enol } \\
\text { Amide }\end{array}$ & $\begin{array}{l}55.08 \\
\text { e }\end{array}$ & $e^{118.32}$ & $\begin{array}{l}25.62 \\
26.20\end{array}$ & $\begin{array}{l}28.41(\mathrm{q}, \mathrm{J}=126.7 \mathrm{~Hz}) ; 52.07(\mathrm{~s}) \\
27.71(\mathrm{q}, \mathrm{J}=125.5 \mathrm{~Hz}) ; 52.07(\mathrm{~s}, \mathrm{ov})\end{array}$ & $173.04(\mathrm{~s})$ & $\begin{array}{l}17.26(\mathrm{~s}) \\
159.52(\mathrm{~s}, \\
\mathrm{w})\end{array}$ \\
\hline & & & THF- $\mathrm{d}_{8}$ & Enol & 54.52 & 117.02 & 25.47 & $28.31(\mathrm{q}, \mathrm{J}=126.6 \mathrm{~Hz}) ; 51.99(\mathrm{~s})$ & $173.18(\mathrm{~s})$ & $173.30(\mathrm{~s})$ \\
\hline & & & & Amide & 46.23 & 114.34 & 25.92 & $27.46(\mathrm{q}, \mathrm{ov}) ; 51.31(\mathrm{~s})$ & $161.54(\mathrm{~s})$ & $159.85(\mathrm{~s})$ \\
\hline & & & & Ion & 70.91 & 116.88 & & $27.31(\mathrm{q}, \mathrm{J}=128.7 \mathrm{~Hz}) ; 51.68(\mathrm{~s})$ & $162.16(\mathrm{~s})$ & $164.35(\mathrm{~s})$ \\
\hline & & & $\mathrm{CD}_{3} \mathrm{CN}$ & Enol & 54.36 & 114.83 & 26.10 & $\begin{array}{l}28.18(\mathrm{q}, \mathrm{J}=125.8 \mathrm{~Hz}) ; 52.48(\mathrm{~s}) ; \\
28.35(\end{array}$ & $173.03(\mathrm{~s})$ & $173.28(\mathrm{~s})$ \\
\hline & & & & Amide & 46.81 & 117.61 & 26.33 & $27.04(\mathrm{q}, \mathrm{ov}) ; 27.19(\mathrm{q}, \mathrm{ov}) ; 51.87(\mathrm{~s})$ & 161.55 & 159.79 \\
\hline & & & & Ion & 70.87 & 116.90 & & $\begin{array}{l}27.36(\mathrm{q}, \mathrm{J}=126.8 \mathrm{~Hz}) ; 27.45(\mathrm{q}, \mathrm{ov}) ; \\
52.27(\mathrm{~s})\end{array}$ & 161.73 & 164.00 \\
\hline & & & DMF- $\mathrm{d}_{7}$ & Ion & 74.88 & 117.65 & 26.05 & $27.52(\mathrm{q}, \mathrm{J}=126.7 \mathrm{~Hz}) ; 51.76(\mathrm{~s})$ & $162.54(\mathrm{~s})$ & $164.44(\mathrm{~s})$ \\
\hline & & & $\mathrm{DMSO}_{-} \mathrm{d}_{6}$ & Enol & 54.5 & 118.45 & 26.56 & $29.32(\mathrm{q}, \mathrm{J}=127.3 \mathrm{~Hz})$ & & $\begin{array}{l}173.03(\mathrm{~s}, \\
\mathrm{w})\end{array}$ \\
\hline & \multirow{8}{*}{$\mathrm{H}$} & \multirow{8}{*}{$\mathrm{C}_{6} \mathrm{H}_{5}$} & & Amide & 46.92 & 115.90 & 26.89 & $28.49(\mathrm{q}, \mathrm{J}=126.3 \mathrm{~Hz}) ; 51.65(\mathrm{~s})$ & $161.69(\mathrm{~s})$ & $160.15(\mathrm{~s})$ \\
\hline \multirow[t]{7}{*}{$\mathrm{H}$} & & & THF-d 8 & Enol & 55.75 & 116.26 & & $\begin{array}{l}121.31(\mathrm{~d}, \mathrm{~J}=162.4 \mathrm{~Hz}) ; 123.79(\mathrm{~d}, \mathrm{~J} \\
=155.3 \mathrm{~Hz}) ; 128.20(\mathrm{~d}, \mathrm{~J}=160.4 \\
\mathrm{Hz}) ; 137.81(\mathrm{t}, \mathrm{J}=8.2 \mathrm{~Hz})\end{array}$ & $\begin{array}{l}171.15(\mathrm{~d} \\
\mathrm{J}=3.2 \mathrm{~Hz})\end{array}$ & $175.16(\mathrm{~s})$ \\
\hline & & & $\mathrm{CD}_{3} \mathrm{CN}$ & Enol & 55.93 & 116.54 & & $\begin{array}{l}122.40(\mathrm{~d}, \mathrm{~J}=163.0 \mathrm{~Hz}) ; 124.96(\mathrm{~d}, \mathrm{~J} \\
=163.5 \mathrm{~Hz}) ; 128.72\left(\mathrm{~d} \text { of d, J } \mathrm{J}_{1}=\right. \\
\left.162.4 \mathrm{~Hz}, \mathrm{~J}_{2}=8.3 \mathrm{~Hz}\right) ; 136.76(\mathrm{t})\end{array}$ & $171.25(\mathrm{~s})$ & $175.08(\mathrm{~s})$ \\
\hline & & & & Amide & 47.05 & e & & $\begin{array}{l}120.12(\mathrm{~d}, \mathrm{~J}=156.4 \mathrm{~Hz}) ; 124.96(\mathrm{~d}, \\
\mathrm{ov}) ; 129.01(\mathrm{~d}, \mathrm{~J}=163.5 \mathrm{~Hz})\end{array}$ & e & e \\
\hline & & & DMF-d 7 & Enol & 55.60 & 116.90 & & $\begin{array}{l}121.96(\mathrm{~d}, \mathrm{~J}=161.8 \mathrm{~Hz}) ; 124.06(\mathrm{~d}, \mathrm{~J} \\
=158.0 \mathrm{~Hz}) ; 128.44(\mathrm{~d}, \mathrm{~J}=158.0 \\
\mathrm{Hz}) ; 138.02(\mathrm{~s})\end{array}$ & $171.43(\mathrm{o})$ & $175.10(\mathrm{~s})$ \\
\hline & & & & Amide & 47.47 & 114.97 & & $\begin{array}{l}119.37(\mathrm{~d}, \mathrm{~J}=160.9 \mathrm{~Hz}) ; 124.06(\mathrm{~d}, \\
\text { ov); } 128.84(\mathrm{~d}, \mathrm{~J}=160.3 \mathrm{~Hz}) ; 138.68 \\
\text { (s) }\end{array}$ & $\begin{array}{l}162.01(\mathrm{~s}, \\
\text { ov) }\end{array}$ & $159.72(\mathrm{~s})$ \\
\hline & & & $\mathrm{DMSO}_{-} \mathrm{d}_{6}$ & Enol & 55.67 & 117.78 & & $\begin{array}{l}122.68(\mathrm{~d}, \mathrm{~J}=160.3 \mathrm{~Hz}) ; 124.56(\mathrm{~d}, \\
\text { ov); } 128.90\left(\mathrm{~d} \text { of d, } \mathrm{J}_{1}=160.8 \mathrm{~Hz}, \mathrm{~J}_{2}\right. \\
=8.3 \mathrm{~Hz}) ; 137.97(\mathrm{~s})\end{array}$ & $171.45(\mathrm{~s})$ & $174.83(\mathrm{~s})$ \\
\hline & & & & Amide & 47.79 & 115.59 & & $119.87(\mathrm{~d}, \mathrm{~J}=163.1 \mathrm{~Hz}) ; 124.69(\mathrm{~d}, \mathrm{~J}$ & $162.55(\mathrm{~s})$ & $159.90(\mathrm{~s})$ \\
\hline
\end{tabular}


$=163.1 \mathrm{~Hz}) ; 129.42\left(\mathrm{~d}\right.$ of $\mathrm{d}, \mathrm{J}_{1}=$

$\left.162.3 \mathrm{~Hz}, \mathrm{~J}_{2}=8.3 \mathrm{~Hz}\right) ; 138.64(\mathrm{~s})$

$\begin{array}{lllll}p-\mathrm{MeO}- & \text { THF- } \mathrm{d}_{8} & \text { Enol } & 55.29 & 116.43\end{array}$

$\mathrm{C}_{6} \mathrm{H}_{4}$

$\begin{array}{llll} & \text { Amide } & \text { e } & \text { e } \\ & & & \\ \mathrm{CD}_{3} \mathrm{CN} & \text { Enol } & 55.48 & 116.67 \\ & \text { Amide } & 47.05 & 114.03 \\ & & & \\ \text { DMF-d }_{7} & \text { Enol } & 55.11 & 116.98 \\ & & & \\ & & & \\ & \text { Amide } & 47.30 & 113.82\end{array}$

DMSO-d $_{6} \quad$ Enol $\quad 55.21 \quad 117.93$

Amide $\quad 47.67 \quad 115.71$

$\begin{array}{lllll}\mathrm{C}_{6} \mathrm{~F}_{5} & \text { THF-d } & \text { Enol } & 56.09 & 115.75\end{array}$

$\mathrm{CD}_{3} \mathrm{CN} \quad$ Enol $\quad 56.30 \quad 116.01$

Amide $\quad 46.01 \quad 113.74$

$\mathrm{DMF}_{-\mathrm{d}} \quad$ Enol $^{\mathrm{h}} \quad 55.80 \quad 116.34$ $54.44(\mathrm{q}, \mathrm{J}=143.5 \mathrm{~Hz}) ; 113.33(\mathrm{~d}, \mathrm{~J}=171.07(\mathrm{~s}) \quad 175.14(\mathrm{~s})$

$159.3 \mathrm{~Hz}) ; 123.36(\mathrm{~d}, \mathrm{~J}=161.6 \mathrm{~Hz})$;

$130.39(\mathrm{~s}) ; 156.77(\mathrm{~s})$

54.44 (ov); 113.51 (d, ov); 119.68 (d,

$\mathrm{J}=161.8 \mathrm{~Hz})$

$55.06(\mathrm{q}) ; 113.81 ; 124.72 ; 129.26 ; \quad 171.31(\mathrm{~s}) \quad 175.05(\mathrm{~s})$

157.29

$55.01(\mathrm{q}) ; 113.91 ; 122.05 ; 129.26$ e

(ov), 157.29 (ov)

$54.94(\mathrm{q}, \mathrm{J}=143.9 \mathrm{~Hz}) ; 113.58(\mathrm{~d}, \mathrm{~J}=171.33(\mathrm{~s}) \quad 175.12(\mathrm{~s})$

$160.1 \mathrm{~Hz}) ; 124.00(\mathrm{~d}, \mathrm{~J}=161.6 \mathrm{~Hz})$;

$130.61(\mathrm{~s}) ; 156.61(\mathrm{~s})$

$54.96(\mathrm{q}, \mathrm{ov}) ; 113.92(\mathrm{~d}, \mathrm{~J}=162.3 \quad 162.55(\mathrm{~s}) \quad 159.28(\mathrm{~s})$

$\mathrm{Hz}) ; 121.00(\mathrm{~d}, \mathrm{~J}=164.2 \mathrm{~Hz}) ; 131.76$

(s); $156.34(\mathrm{~s})$

$55.68(\mathrm{q}, \mathrm{J}=144.1 \mathrm{~Hz}) ; 114.10(\mathrm{~d}, \mathrm{~J}=171.38(\mathrm{~s}) \quad 174.91(\mathrm{~s})$

$160.1 \mathrm{~Hz}) ; 124.76(\mathrm{~d}, \mathrm{~J}=163.8 \mathrm{~Hz})$;

$130.65(\mathrm{~s}) ; 156.64(\mathrm{~s})$

$55.68(\mathrm{ov}) ; 114.52(\mathrm{~d}, \mathrm{~J}=160.8 \mathrm{~Hz}) ; \quad 162.71(\mathrm{~s}) \quad 159.41(\mathrm{~d}$,

$121.52(\mathrm{~d}, \mathrm{~J}=163.8 \mathrm{~Hz}) ; 131.73(\mathrm{t}) ; \quad \mathrm{J}=6.0 \mathrm{~Hz})$

$156.36(\mathrm{~s})$

$112.47(\mathrm{t}, \mathrm{J}=12.8 \mathrm{~Hz}) ; 135.91(\mathrm{~m}) ; \quad 171.42(\mathrm{~s}) \quad 174.84(\mathrm{~s})$

$139.27(\mathrm{~m}) ; 141.79(\mathrm{~m}) ; 142.45(\mathrm{~m})$;

$145.67(\mathrm{~m})$

$111.34(\mathrm{t}, \mathrm{J}=14.3 \mathrm{~Hz}) ; 136.50(\mathrm{t}, \mathrm{J}=171.25(\mathrm{~d}, \quad 174.69$ (s) $13.2 \mathrm{~Hz}) ; 139.16(\mathrm{~m}) ; 141.70(\mathrm{t}, \mathrm{J}=\mathrm{J}=4.0 \mathrm{~Hz})$

$13.8 \mathrm{~Hz}) ; 142.60(\mathrm{~m}) ; 145.08(\mathrm{~m})$

111.34 (t, ov); 136.50 (t, ov); $139.16 \quad 162.06(\mathrm{~s}) \quad 160.04(\mathrm{~s})$

(m, ov); $141.70(\mathrm{t}, \mathrm{ov}) ; 142.60(\mathrm{~m}$,

ov); 145.08 (m, ov)

$112.70(\mathrm{~m}) ; 135.92(\mathrm{~m}) ; 139.23(\mathrm{~m})$

$171.65(\mathrm{~s}) \quad 174.60(\mathrm{~s})$ 


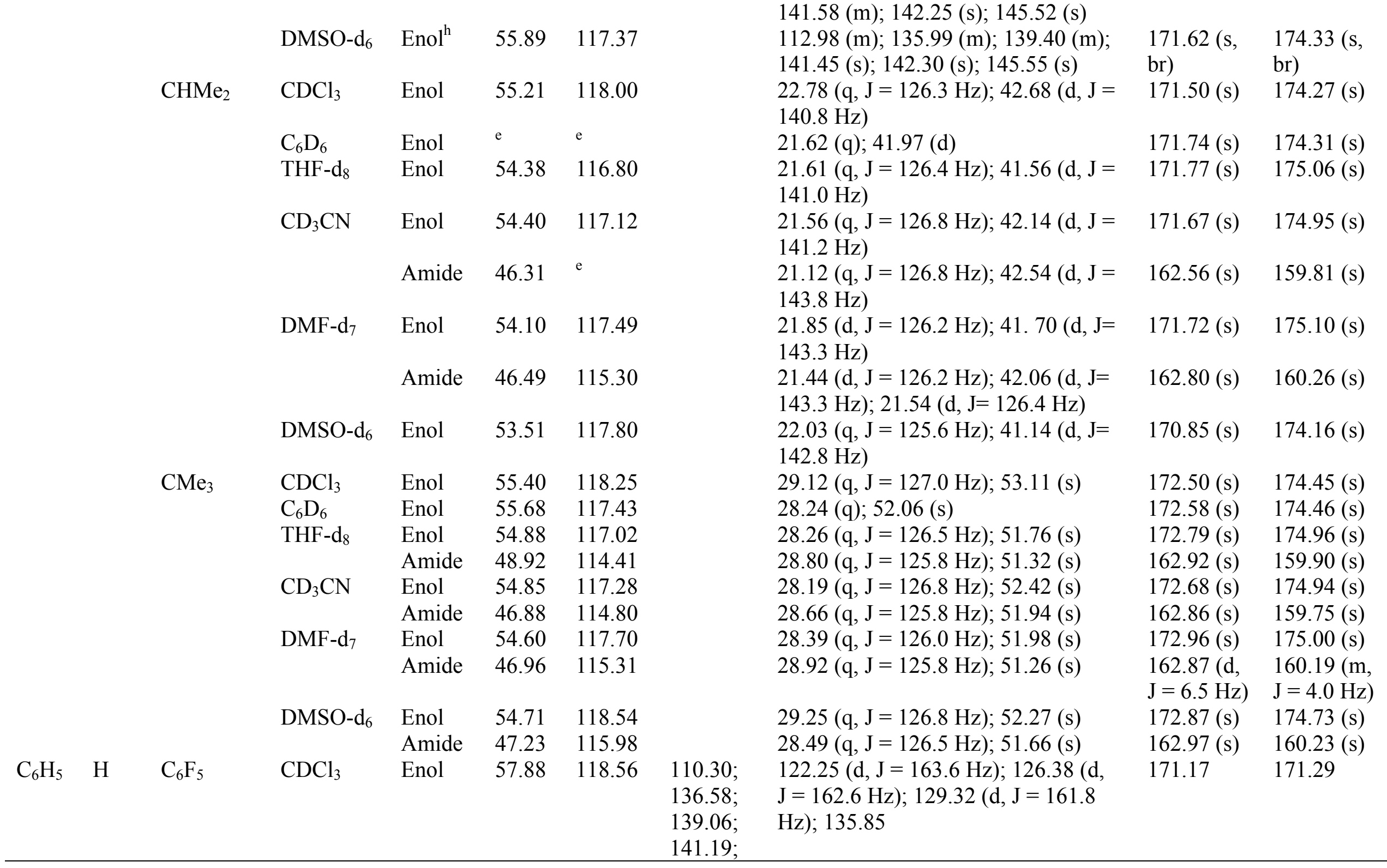




\begin{tabular}{|c|c|c|c|c|c|c|c|}
\hline $\mathrm{C}_{6} \mathrm{D}_{6}$ & Enol & 57.87 & 116.62 & $\begin{array}{l}110.38 ; \\
136.30 ; \\
138.82 ; \\
141.77 ; \\
142.43 ; \\
145.04\end{array}$ & $\begin{array}{l}121.92(\mathrm{~d}, \mathrm{~J}=163.1 \mathrm{~Hz}) ; 125.68(\mathrm{~d}, \\
\mathrm{J}=162.1 \mathrm{~Hz}) ; 128.77(\mathrm{~d}, \mathrm{~J}=163.0 \\
\mathrm{Hz}) ; 135.03(\mathrm{t})\end{array}$ & $\begin{array}{l}171.06(\mathrm{~d} \\
\mathrm{J}=4.3 \mathrm{~Hz})\end{array}$ & $\begin{array}{l}171.67(\mathrm{~d}, \\
\mathrm{J}=4.8 \mathrm{~Hz})\end{array}$ \\
\hline \multirow[t]{2}{*}{ THF-d 8} & Enol & 57.50 & 115.01 & $\begin{array}{l}111.91 ; \\
136.41 ; \\
138.93 ; \\
141.68 \\
142.74 ; \\
145.35\end{array}$ & $\begin{array}{l}123.16(\mathrm{~d}, \mathrm{~J}=162.7 \mathrm{~Hz}) ; 125.40(\mathrm{~d}, \\
\mathrm{J}=161.9 \mathrm{~Hz}) ; 128.47(\mathrm{~d}, \mathrm{~J}=163.0 \\
\mathrm{Hz}) ; 136.20(\mathrm{t})\end{array}$ & 171.72 & 172.40 \\
\hline & Amide & 47.24 & e & $\begin{array}{l}111.91 \\
\text { (ov); } \\
136.41 \\
\text { (ov); } \\
138.93 \\
\text { (ov); } \\
141.68 \\
\text { (ov); } \\
142.74 \\
\text { (ov); } \\
145.35 \\
\text { (ov) }\end{array}$ & $119.80 ; 124.43 ; 128.56 ; 136.43$ & 159.92 & 158.26 \\
\hline $\mathrm{CD}_{3} \mathrm{CN}$ & Enol & 57.51 & 115.51 & $\begin{array}{l}111.36 \\
136.64 \\
139.07 \\
141.96 \\
142.77 \\
145.15\end{array}$ & $\begin{array}{l}123.76(\mathrm{~d}, \mathrm{~J}=162.1 \mathrm{~Hz}) ; 126.23(\mathrm{~d}, \mathrm{~J} \\
=161.9 \mathrm{~Hz}) ; 128.91(\mathrm{~d}, \mathrm{~J}=163.2 \\
\mathrm{Hz}) ; 135.39(\mathrm{t})\end{array}$ & 160.62 & 158.92 \\
\hline & Amide & 47.28 & 113.43 & 111.36 & $120.28(\mathrm{~d}, \mathrm{~J}=163.0 \mathrm{~Hz}) ; 125.29(\mathrm{~d}$, & 159.92 & 158.43 \\
\hline
\end{tabular}




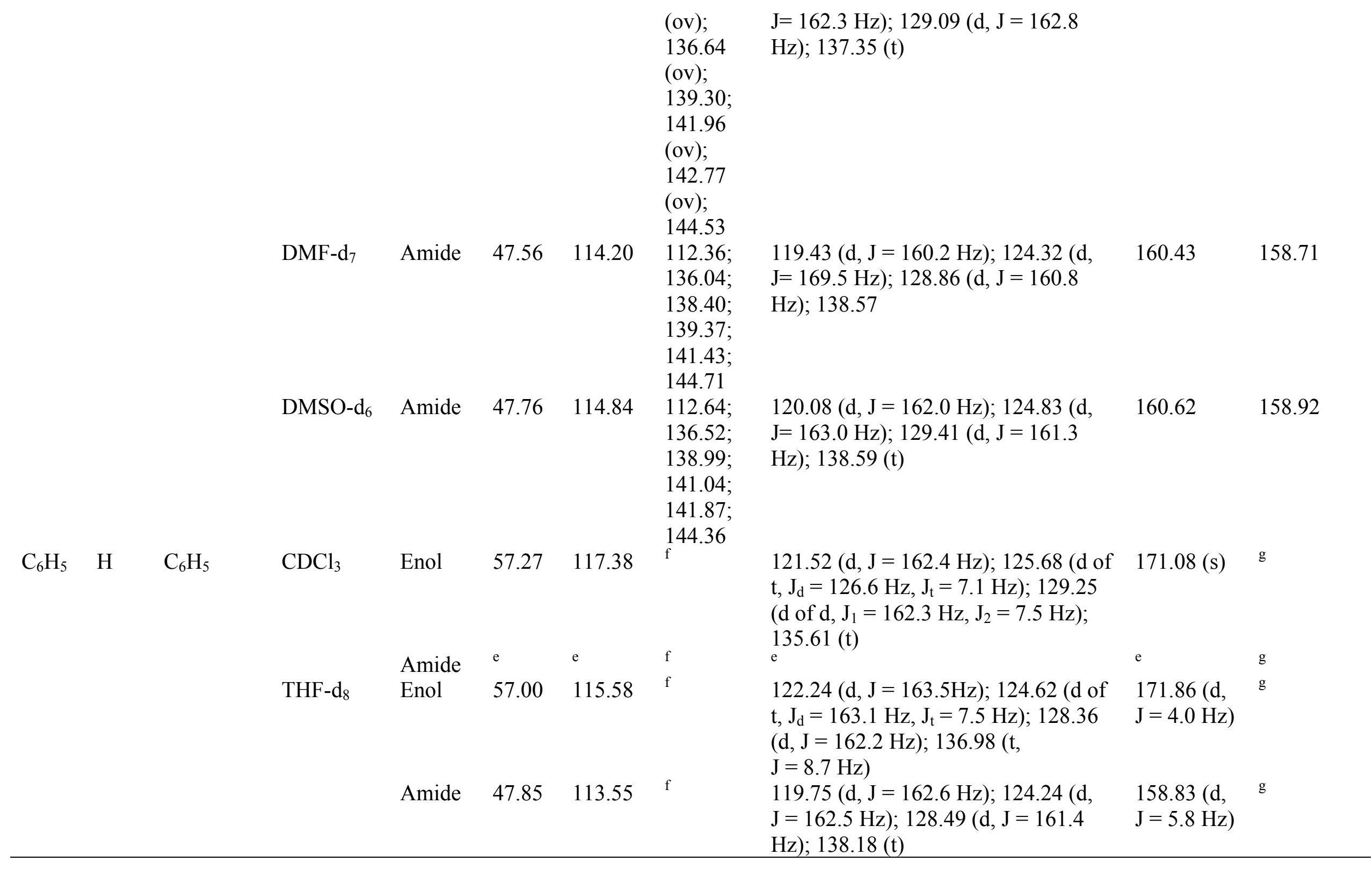




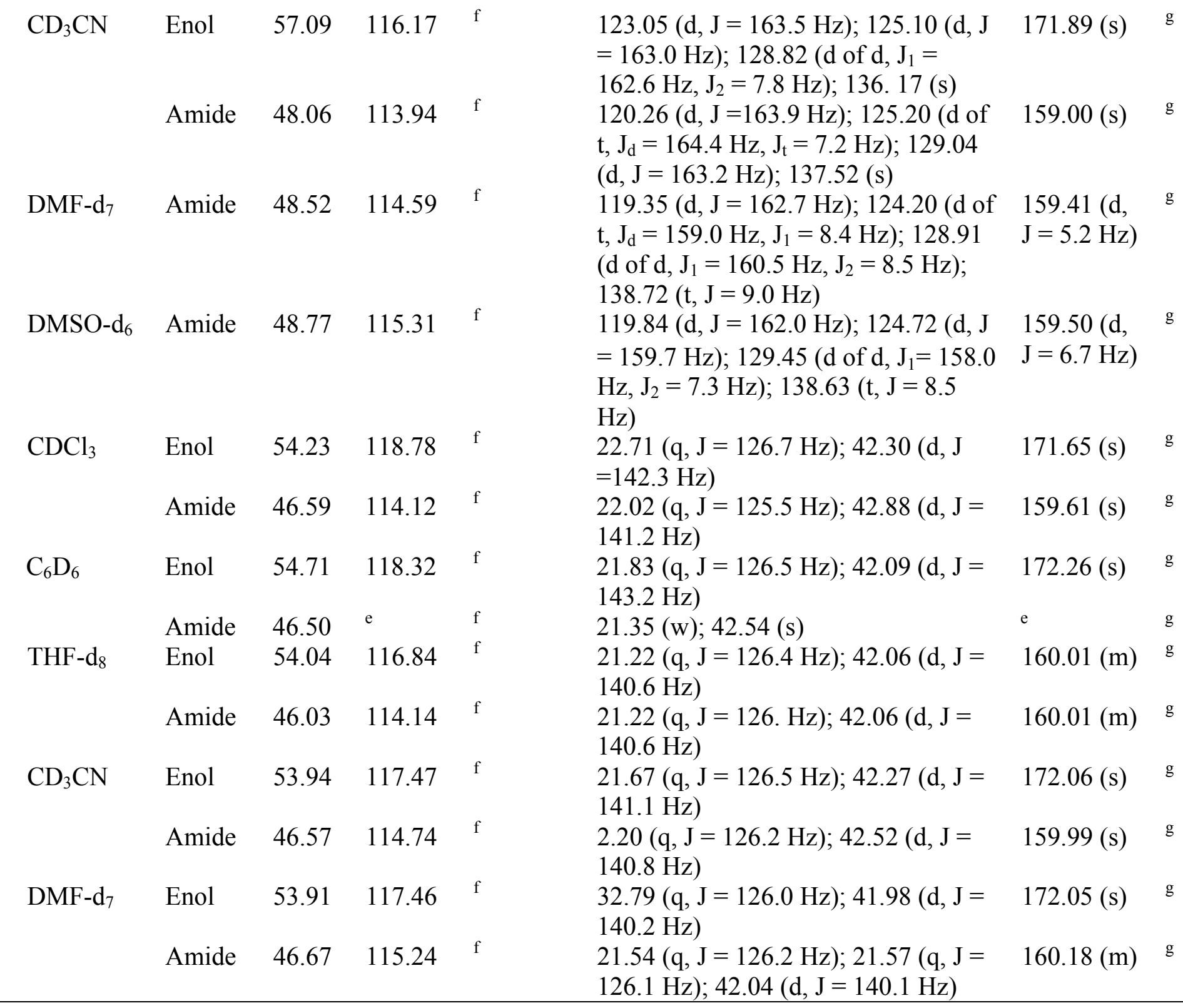




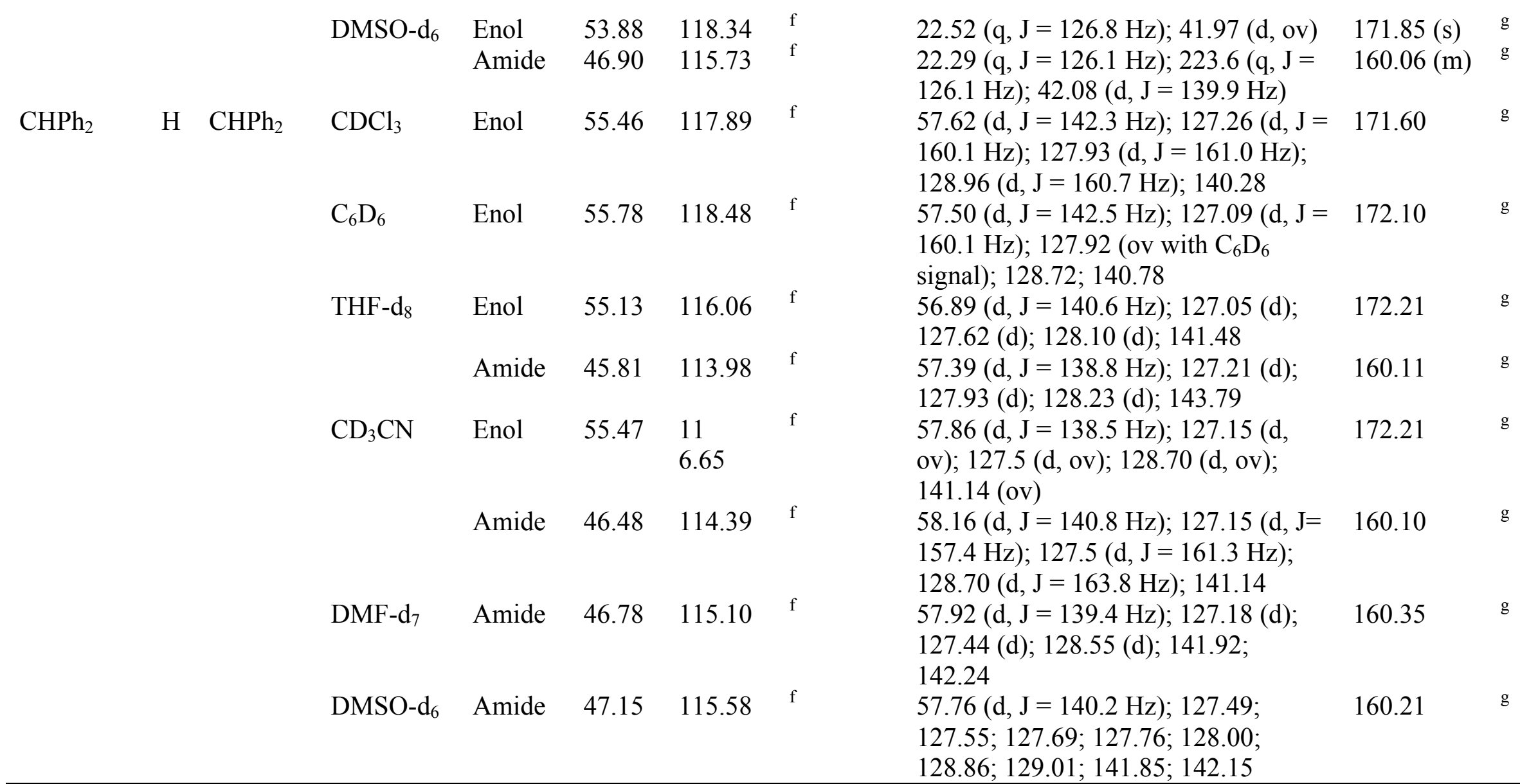

${ }^{\mathrm{a}}$ Abbreviation: $\mathrm{s}=$ singlet, $\mathrm{d}=$ doublet, $\mathrm{t}=$ triplet, $\mathrm{q}=$ quartet $\mathrm{m}=$ multiplet, $\mathrm{w}=$ weak, $\mathrm{vw}=$ very weak, ov $=$ overlaps. $^{\mathrm{b}}$ Singlet for the enol; doublet, $\mathrm{J}=136.4-138.9 \mathrm{~Hz}$ for the amide. ${ }^{\mathrm{c}}$ Singlet for the enol; doublet, $\mathrm{J}=10.6-11.3 \mathrm{~Hz}$ for the amide. ${ }^{\mathrm{d}}$ Quartet for the enol, $\mathrm{J}=135.3-$ $140.1 \mathrm{~Hz}$; Two quartets for the amide: for the higher field signal, $\mathrm{J}=138.8-141.9$. For the lower field signal $\mathrm{J}=137.5-140.0 \mathrm{~Hz}$. ${ }^{\mathrm{J}} \mathrm{Not}$ observed. ${ }^{\mathrm{f}} \mathrm{R}^{3}=\mathrm{R}^{1} .{ }^{\mathrm{g}} \mathrm{C}_{-}=\mathrm{C}=\mathrm{O} .{ }^{\mathrm{h}}$ The amide was not observed. 
Table S4. IR Spectral Data (in $\left.\mathrm{cm}^{-1}\right)$ of $\mathrm{R}^{3} \mathrm{NHCOCH}(\mathrm{CN}) \mathrm{CONR}^{1} \mathrm{R}^{2} / \mathrm{R}^{3} \mathrm{NHC}(\mathrm{OH})=\mathrm{C}(\mathrm{CN}) \mathrm{CONR} \mathrm{R}^{2} / \mathrm{R}^{3} \mathrm{NHCOC}(\mathrm{CN})=\mathrm{C}(\mathrm{OH}) \mathrm{NR} \mathrm{R}^{2}(\mathbf{4} / \mathbf{5} / \mathbf{6})$ System in $\mathrm{CHCl}_{3}$ at Room Temperature

\begin{tabular}{|c|c|c|c|c|c|c|}
\hline $\mathrm{R}^{3}$ & $\mathrm{R}^{1}$ & $\mathrm{R}^{2}$ & Comp. & $v_{\mathrm{CN}}$ & $v_{\mathrm{CO}}$ & $v_{\mathrm{NH}, \mathrm{OH}}$ \\
\hline $\mathrm{Ph}$ & $\mathrm{Me}$ & $\mathrm{Me}$ & $4 \mathbf{a} / 5 \mathbf{a} / 6 \mathbf{a}$ & 2188,2255 & 1654,1700 & 3409,3299 \\
\hline$p$-An & $\mathrm{Me}$ & $\mathrm{Me}$ & $4 b / 5 b / 6 b$ & 2187,2255 & 1604,1655 & 3410,3293 \\
\hline $\mathrm{C}_{6} \mathrm{~F}_{5}$ & $\mathrm{Me}$ & $\mathrm{Me}$ & $4 c / 5 c / 6 c$ & 2194 & 1648,1717 & 3403,3252 \\
\hline$i-\operatorname{Pr}$ & $\mathrm{Me}$ & $\mathrm{Me}$ & $4 d / 5 d / 6 d$ & 2185,2254 & 1533,1666 & 3418,3220 (br), 3066 \\
\hline$t$-Bu & $\mathrm{Me}$ & $\mathrm{Me}$ & $4 e / 5 e / 6 e$ & 2183,2254 & 1655 & $3419,3307,3080$ \\
\hline $\mathrm{Ph}$ & $\mathrm{H}$ & $\mathrm{Me}$ & $4 g / 5 g / 6 g$ & 2190 & 1538,1594 & 3320 \\
\hline$p$-An & $\mathrm{H}$ & $\mathrm{Me}$ & $4 h / 5 h / 6 h$ & 2189,2203 & $1511, .1648$ & 3322 \\
\hline $\mathrm{C}_{6} \mathrm{~F}_{5}$ & $\mathrm{H}$ & $\mathrm{Me}$ & $4 i / 5 i / 6 i$ & 2197 & 1613,1644 & $3435,3407,3331$ \\
\hline$i-\operatorname{Pr}$ & $\mathrm{H}$ & $\mathrm{Me}$ & $4 \mathbf{j} / \mathbf{5 j} / \mathbf{6 j}$ & 2188 & 1591,1699 & $3442,3420,3336$ \\
\hline$t-\mathrm{Bu}$ & $\mathrm{H}$ & $\mathrm{Me}$ & $4 k / 5 k / 6 k$ & 2169,2253 & 1542,1716 & 3420,3347 \\
\hline$p$-An & $\mathrm{H}$ & $\mathrm{H}$ & $4 m / 5 m / 6 m$ & 2194 & 1670 & 3420 \\
\hline $\mathrm{C}_{6} \mathrm{~F}_{5}$ & $\mathrm{H}$ & $\mathrm{H}$ & $4 n / 5 n / 6 n$ & 2206 & 1648 & 3481,3420 (br), 3356, 3302 \\
\hline$i-\operatorname{Pr}$ & $\mathrm{H}$ & $\mathrm{H}$ & $40 / 50 / 60$ & 2201 & 1578,1647 & $3415,3336,3235$ \\
\hline$t-\mathrm{Bu}$ & $\mathrm{H}$ & $\mathrm{H}$ & $4 p / 5 p / 6 p$ & 2192 & $1584,1698(w)$ & $3524,3483,3416$ (br) \\
\hline $\mathrm{Ph}$ & $\mathrm{H}$ & $\mathrm{Ph}$ & $4 r / 5 r / 6 r$ & 2194 & $1601,1619,1686$ & 3409,3296 \\
\hline$i-\operatorname{Pr}$ & $\mathrm{H}$ & $i$-Pr & $4 s / 5 s / 6 s$ & $2187,2259(\mathrm{w})$ & 1697 & 3420 (br), 3327 \\
\hline
\end{tabular}


Table S5. Calculated (B3LYP/6-31G**) energy differences for regioisomeric enols.

$$
\text { By }
$$

More stable

$\mathrm{kcal} / \mathrm{mol}$

Than

\begin{tabular}{|c|c|c|}
\hline$p-\mathrm{AnNHC}(\mathrm{OH})=\mathrm{C}(\mathrm{CN}) \mathrm{CONH}_{2}$ & 0.18 & $p-\mathrm{AnNHCOC}(\mathrm{CN})=\mathrm{C}(\mathrm{OH}) \mathrm{NH}_{2}$ \\
\hline $\mathrm{H}_{2} \mathrm{NC}(\mathrm{OH})=\mathrm{C}(\mathrm{CN}) \mathrm{CONHC}_{6} \mathrm{~F}_{5}$ & 0.6 & $\mathrm{H}_{2} \mathrm{NCOC}(\mathrm{CN})=\mathrm{C}(\mathrm{OH}) \mathrm{NHC}_{6} \mathrm{~F}_{5}$ \\
\hline $\mathrm{Me}_{2} \mathrm{NC}(\mathrm{OH})=\mathrm{C}(\mathrm{CN}) \mathrm{CONHC}_{6} \mathrm{~F}_{5}$ & 0.7 & $\mathrm{Me}_{2} \mathrm{NCOC}(\mathrm{CN})=\mathrm{C}(\mathrm{OH}) \mathrm{NHC}_{6} \mathrm{~F}_{5}$ \\
\hline $\mathrm{H}_{2} \mathrm{NC}(\mathrm{OH})=\mathrm{C}(\mathrm{CN}) \mathrm{CONHPr}-i$ & 0.7 & $\mathrm{H}_{2} \mathrm{NCOC}(\mathrm{CN})=\mathrm{C}(\mathrm{OH}) \mathrm{NHPr}-i$ \\
\hline$i-\mathrm{PrNHC}(\mathrm{OH})=\mathrm{C}(\mathrm{CN}) \mathrm{CONHMe}$ & 0.02 & $i-\operatorname{PrNHCOC}(\mathrm{CN})=\mathrm{C}(\mathrm{OH}) \mathrm{NHMe}$ \\
\hline $\mathrm{Me}_{2} \mathrm{NC}(\mathrm{OH})=\mathrm{C}(\mathrm{CN}) \mathrm{CONHPr}-i$ & 0.09 & $\mathrm{Me}_{2} \mathrm{NCOC}(\mathrm{CN})=\mathrm{C}(\mathrm{OH}) \mathrm{NHPr}-i$ \\
\hline $\mathrm{Me}_{2} \mathrm{NC}(\mathrm{OH})=\mathrm{C}(\mathrm{CN}) \mathrm{CONHPh}$ & $?$ & $\begin{array}{l}\mathrm{Me}_{2} \mathrm{NCOC}(\mathrm{CN})=\mathrm{C}(\mathrm{OH}) \mathrm{NHPh} \text { is } \\
\text { not a minimum on the PES }\end{array}$ \\
\hline
\end{tabular}

Table S6. Analytical Data, $m p\left({ }^{\circ} \mathrm{C}\right)$ and Yield (\%) for $\mathbf{4 / 5 / 6}$ Systems

Compound

No. $\quad \mathrm{R}^{3} \quad$ Formula $\quad \mathrm{mp},{ }^{\circ} \mathrm{C} \quad$ yield, \%

Calculated $\%$

$\mathrm{C} \quad \mathrm{H} \quad \mathrm{N}$

Found \%

$\mathrm{C} \quad \mathrm{H} \quad \mathrm{N}$

\begin{tabular}{|l|l|l|c|c|c|c|c|c|c|c|}
\hline $\mathbf{4 a} / \mathbf{5 a} / \mathbf{6 a}$ & $\mathrm{Ph}$ & $\mathrm{C}_{12} \mathrm{H}_{13} \mathrm{~N}_{3} \mathrm{O}_{2}$ & $124-126$ & 55 & 62.34 & 5.63 & 18.18 & 61.97 & 5.94 & 18.11 \\
\hline $\mathbf{4 b} / \mathbf{5 b} / \mathbf{6 b}$ & $p-\mathrm{MeOC}_{6} \mathrm{H}_{4}$ & $\mathrm{C}_{13} \mathrm{H}_{15} \mathrm{~N}_{3} \mathrm{O}_{3}$ & 134 & 52 & 59.77 & 5.75 & 16.09 & 59.55 & 5.78 & 16.16 \\
\hline $\mathbf{4 c / 5} / \mathbf{6 c}$ & $\mathrm{C}_{6} \mathrm{~F}_{5}$ & $\mathrm{C}_{12} \mathrm{H}_{8} \mathrm{~F}_{5} \mathrm{~N}_{3} \mathrm{O}_{2}$ & 138 & 49 & 44.86 & 2.49 & 13.08 & 44.89 & 2.47 & 12.89 \\
\hline $\mathbf{4 d} / \mathbf{5 d} / \mathbf{6 d}$ & $i-\mathrm{Pr}$ & $\mathrm{C}_{9} \mathrm{H}_{15} \mathrm{~N}_{3} \mathrm{O}_{2}$ & $114-115$ & 20 & 54.82 & 7.61 & 21.32 & 54.82 & 7.68 & 21.43 \\
\hline $\mathbf{4 e} / \mathbf{5 e} / \mathbf{6 e}$ & $t-\mathrm{Bu}$ & $\mathrm{C}_{10} \mathrm{H}_{17} \mathrm{~N}_{3} \mathrm{O}_{2}$ & $142-143$ & 25 & 56.87 & 8.06 & 19.91 & 56.86 & 8.33 & 19.9 \\
\hline
\end{tabular}




\begin{tabular}{|c|c|c|c|c|c|c|c|c|c|c|}
\hline $4 f / 5 f / 6 f$ & $\mathrm{CPh}_{3}{ }^{\mathrm{a}}$ & $\mathrm{C}_{25} \mathrm{H}_{23} \mathrm{~N}_{3} \mathrm{O}_{2}$ & $157-9$ & 74 & 75.57 & 5.79 & 10.58 & 77.27 & 5.90 & 9.54 \\
\hline $4 g / 5 g / 6 g$ & $\mathrm{Ph}$ & $\mathrm{C}_{11} \mathrm{H}_{11} \mathrm{~N}_{3} \mathrm{O}_{2}$ & 140 & 59 & 60.83 & 5.07 & 19.35 & 60.53 & 5.17 & 19.48 \\
\hline $4 h / 5 h / 6 h$ & $p-\mathrm{MeOC}_{6} \mathrm{H}_{4}$ & $\mathrm{C}_{12} \mathrm{H}_{13} \mathrm{~N}_{3} \mathrm{O}_{3}$ & 148 & 64 & 58.30 & 5.26 & 17.00 & 58.31 & 5.29 & 16.77 \\
\hline $4 i / 5 i / 6 i$ & $\mathrm{C}_{6} \mathrm{~F}_{5}$ & $\mathrm{C}_{11} \mathrm{H}_{6} \mathrm{~F}_{5} \mathrm{~N}_{3} \mathrm{O}_{2}$ & 170 & 51 & 43.00 & 1.95 & 13.68 & 43.28 & 2.17 & 13.28 \\
\hline $4 j / 5 j / 6 j$ & $i-\operatorname{Pr}$ & $\mathrm{C}_{8} \mathrm{H}_{13} \mathrm{~N}_{3} \mathrm{O}_{2}$ & $133-4$ & 64 & 52.46 & 7.10 & 22.95 & 52.74 & 7.02 & 23.13 \\
\hline $4 k / 5 k / 6 k$ & $t-\mathrm{Bu}$ & $\mathrm{C}_{9} \mathrm{H}_{15} \mathrm{~N}_{3} \mathrm{O}_{2}$ & 138 & 67 & 54.82 & 7.61 & 21.32 & 54.81 & 7.74 & 21.32 \\
\hline $4 l / 5 l / 6 I$ & $\mathrm{Ph}$ & $\mathrm{C}_{10} \mathrm{H}_{9} \mathrm{~N}_{3} \mathrm{O}_{2}$ & $176-7$ & 28 & 59.11 & 4.43 & 20.69 & 59.51 & 4.56 & 20.29 \\
\hline $4 \mathrm{~m} / 5 \mathrm{~m} / 6 \mathrm{~m}$ & $p-\mathrm{MeOC}_{6} \mathrm{H}_{4}$ & $\mathrm{C}_{11} \mathrm{H}_{11} \mathrm{~N}_{3} \mathrm{O}_{3}$ & 172 & 62 & 56.65 & 4.72 & 18.03 & 57.39 & 4.89 & 17.15 \\
\hline $4 n / 5 n / 6 n$ & $\mathrm{C}_{6} \mathrm{~F}_{5}$ & $\mathrm{C}_{10} \mathrm{H}_{4} \mathrm{~F}_{5} \mathrm{~N}_{3} \mathrm{O}_{2}$ & 168 & 66 & 40.95 & 1.47 & 14.31 & 40.99 & 1.38 & 14.01 \\
\hline $40 / 50 / 60$ & $i-\operatorname{Pr}$ & $\mathrm{C}_{7} \mathrm{H}_{11} \mathrm{~N}_{3} \mathrm{O}_{2}$ & 166 & 39 & 49.70 & 6.51 & 24.85 & 49.79 & 6.61 & 24.92 \\
\hline $4 p / 5 p / 6 p$ & $t-\mathrm{Bu}$ & $\mathrm{C}_{8} \mathrm{H}_{13} \mathrm{~N}_{3} \mathrm{O}_{2}$ & 162 & 26 & 52.46 & 7.10 & 22.95 & 52.65 & 7.17 & 22.65 \\
\hline $4 q / 5 q / 6 q$ & $\mathrm{C}_{6} \mathrm{~F}_{5}$ & $\mathrm{C}_{16} \mathrm{H}_{8} \mathrm{~F}_{5} \mathrm{~N}_{3} \mathrm{O}_{2}$ & $187-188$ & 58 & 52.03 & 2.17 & 11.38 & 51.75 & 2.31 & 11.21 \\
\hline $4 r / 5 r / 6 r$ & $\mathrm{Ph}$ & $\mathrm{C}_{16} \mathrm{H}_{13} \mathrm{~N}_{3} \mathrm{O}_{2}$ & $189-190$ & 58 & 68.82 & 4.66 & 15.05 & 68.64 & 4.65 & 14.65 \\
\hline $4 s / 5 s / 6 s$ & $i-\operatorname{Pr}$ & $\mathrm{C}_{10} \mathrm{H}_{17} \mathrm{~N}_{3} \mathrm{O}_{2}$ & 178 & 51 & 56.87 & 8.06 & 19.90 & 56.42 & 8.01 & 19.43 \\
\hline $4 t / 5 t / 6 t$ & $\mathrm{CHPh}_{2}$ & $\mathrm{C}_{30} \mathrm{H}_{25} \mathrm{~N}_{3} \mathrm{O}_{2}$ & 182 & 47 & 78.43 & 5.45 & 9.15 & 78.48 & 5.46 & 8.82 \\
\hline
\end{tabular}

${ }^{a}$ decomposed during recrystallization

${ }^{1} \mathrm{H}$ NMR of a mixture of $\mathrm{R}^{3} \mathrm{NHCOCH}(\mathrm{CN}) \mathrm{CONR}^{1} \mathrm{R}^{2}(4)$ and their enols $(5 / 6)$ in several solvents. 
${ }^{1} \mathrm{H}$ NMR of a mixture of $\mathrm{PhNHCOCH}(\mathrm{CN}) \mathrm{CONMe}_{2}$ and its enol in $\mathrm{CDCl}_{3}$

$1 H-N M R$
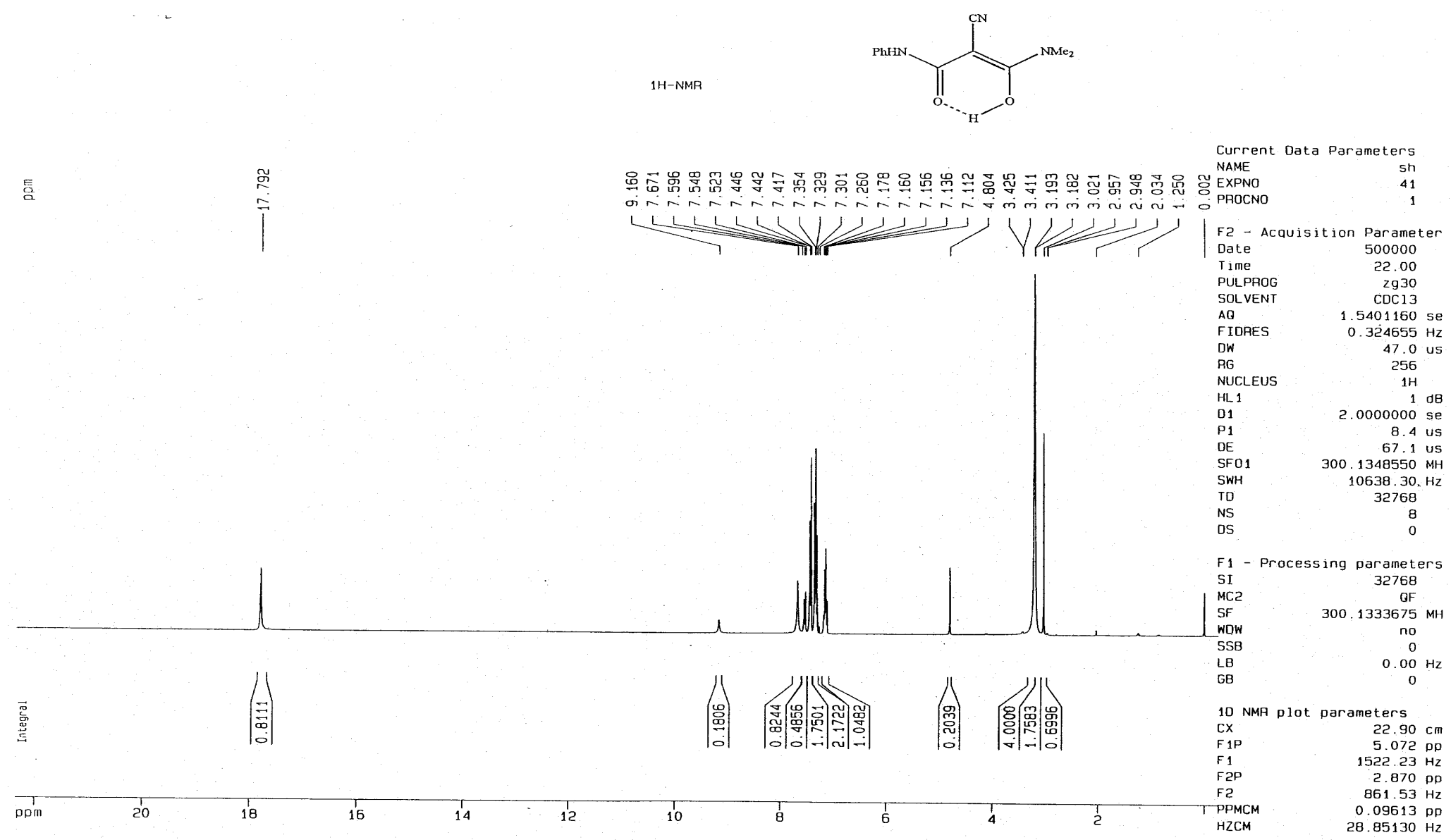

${ }^{1} \mathrm{H}$ NMR of a mixture of $p$ - $\mathrm{AnNHCOCH}(\mathrm{CN}) \mathrm{CONMe}_{2}$ and its enol in $\mathrm{CD}_{3} \mathrm{CN}$ 


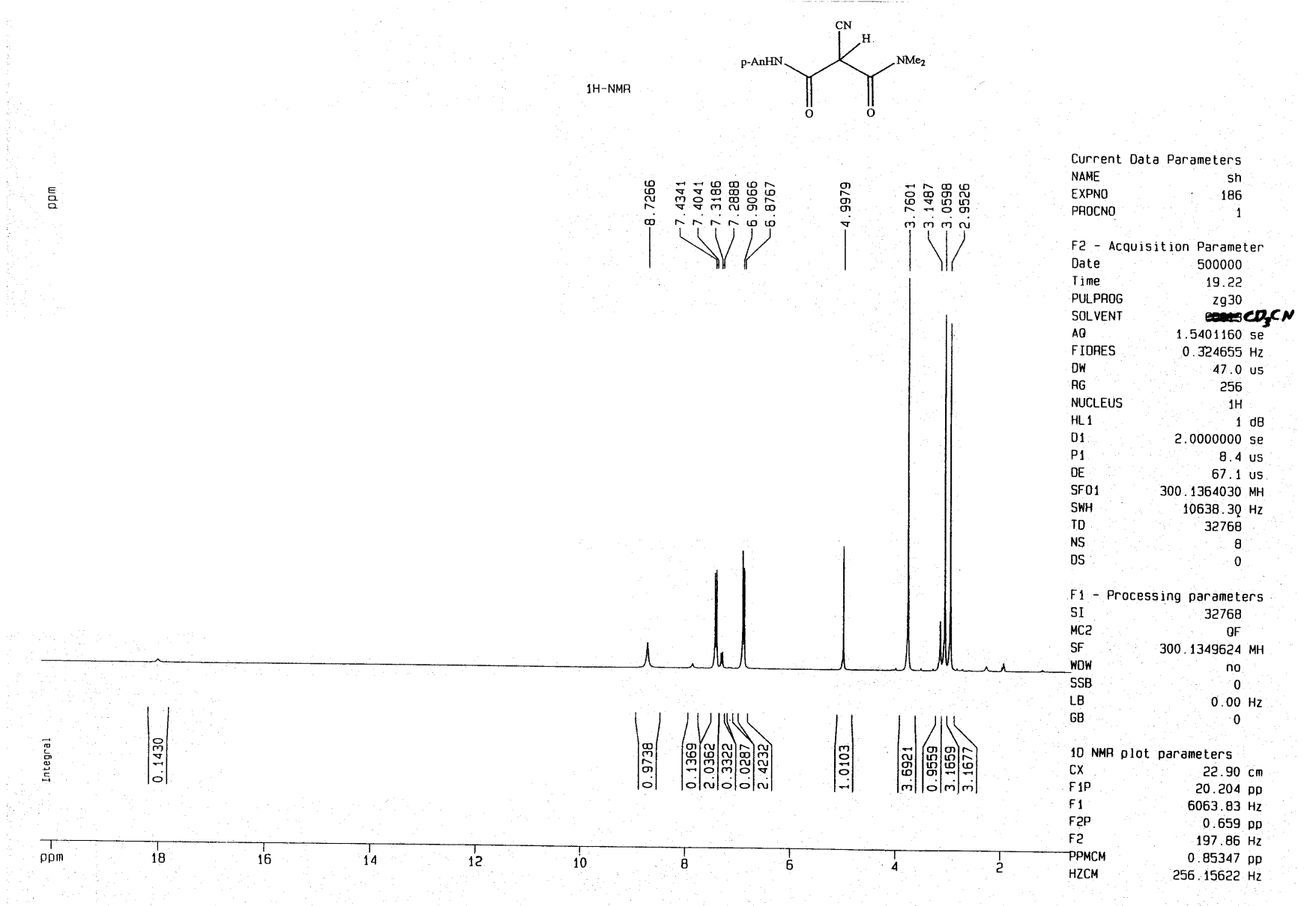

${ }^{1} \mathrm{H}$ NMR of a mixture of $\mathrm{C}_{6} \mathrm{~F}_{5} \mathrm{NHCOCH}(\mathrm{CN}) \mathrm{CONMe}_{2}$ and its enol in $\mathrm{CD}_{3} \mathrm{CN}$ 


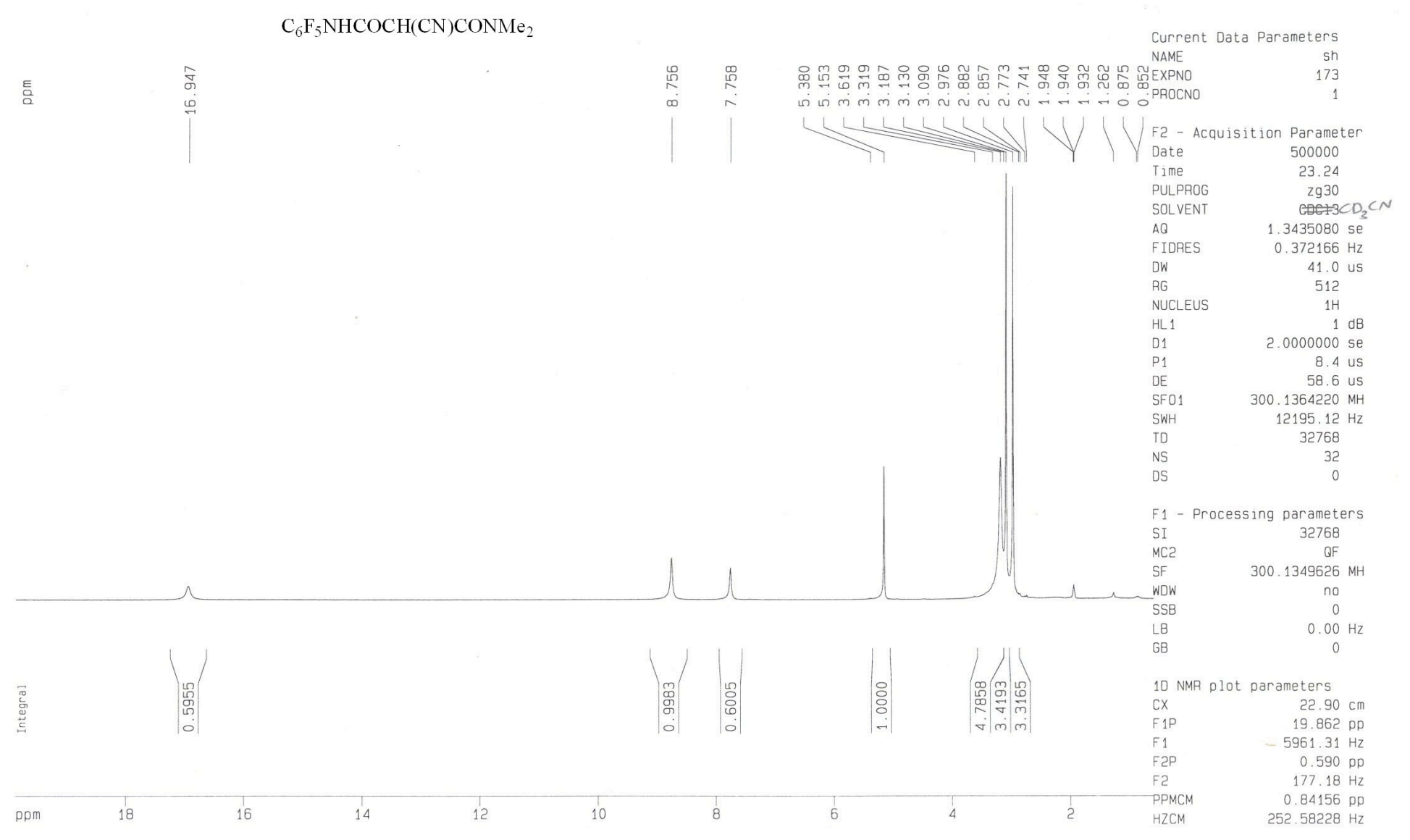

${ }^{1} \mathrm{H}$ NMR of a mixture of $i-\mathrm{PrNHCOCH}(\mathrm{CN}) \mathrm{CONMe}_{2}$ and its enol in $\mathrm{CDCl}_{3}$ 


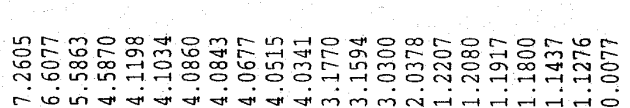

Current Data Parameters
NAME

EXPNO

F2 - Acquisition Parameter

F2 - Acquisition Parameters
Date_

$\begin{array}{lr}\text { Time } & 13.37 \\ \text { INSTROM } & \text { drx400 }\end{array}$

PROBHD
PULPROG 5 min Multinu

TD

$\begin{array}{lr}\text { SOLVENT } & \text { CDC13 } \\ \text { NS } & 32 \\ \text { DS } & 0 \\ \text { SHH } & 14005.603\end{array}$

\begin{tabular}{lr} 
SWH & $14005.603 \mathrm{~Hz}$ \\
PIDRES & 0.217309 \\
\hline
\end{tabular}

$\begin{array}{lr}\text { PIDRES } & 0.213789 \mathrm{~Hz} \\ \mathrm{AQ} & 2.3396852 \mathrm{sec} \\ \mathrm{RG} & 362 \mathrm{~T}\end{array}$

$\begin{array}{ll}\mathrm{RG} & 362 \\ \mathrm{DW} & 35.700 \mathrm{usec} \\ \mathrm{DE} & 10.00 \text { usec } \\ \mathrm{DE} & 300.0 \mathrm{~K}\end{array}$

D1 $\quad 2.00000000 \mathrm{sec}$

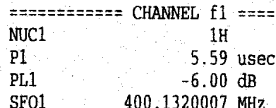

$\begin{array}{lr}\text { F2 - Processing parameters } \\ \text { SI } & 32768 \\ \text { SF } & 400.1300091 \\ \text { MHz }\end{array}$

$\begin{array}{lc}\text { WDW } & \text { no } \\ \text { SSB } & 0 \\ \text { LB } & 0.00 \mathrm{~Hz}\end{array}$

$\mathrm{LB}$
$\mathrm{GB}$
$\mathrm{PC}$

1D NMR plot parameters

CX
CIP
FIP

$\begin{array}{ll}\text { P1P } & 20.499 \mathrm{pp} \\ \mathrm{P1} & 8202.37 \mathrm{~Hz}\end{array}$

$\begin{array}{ll}\text { F2P } & -0.700 \mathrm{ppm} \\ \text { F2 } & -280.19 \mathrm{~Hz}\end{array}$

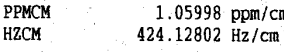

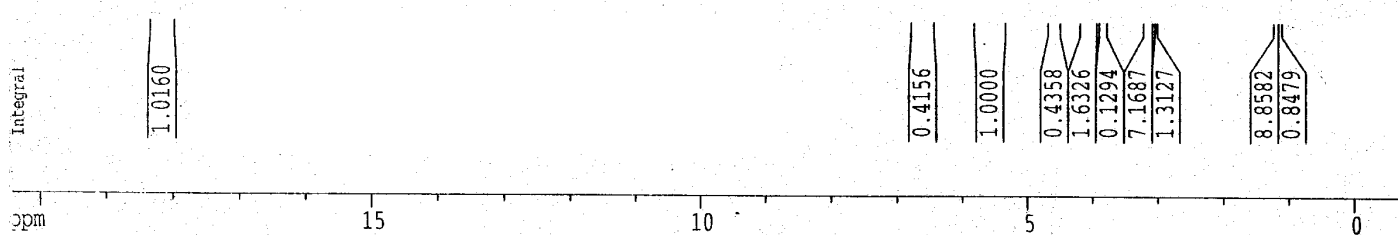


${ }^{1} \mathrm{H}$ NMR of a mixture of $t-\mathrm{BuNHCOCH}(\mathrm{CN}) \mathrm{CONMe}_{2}$ and its enol in $\mathrm{CDCl}_{3}$

$t$ - $\mathrm{BuNHCOCH}(\mathrm{CN}) \mathrm{CONMe}_{2}$

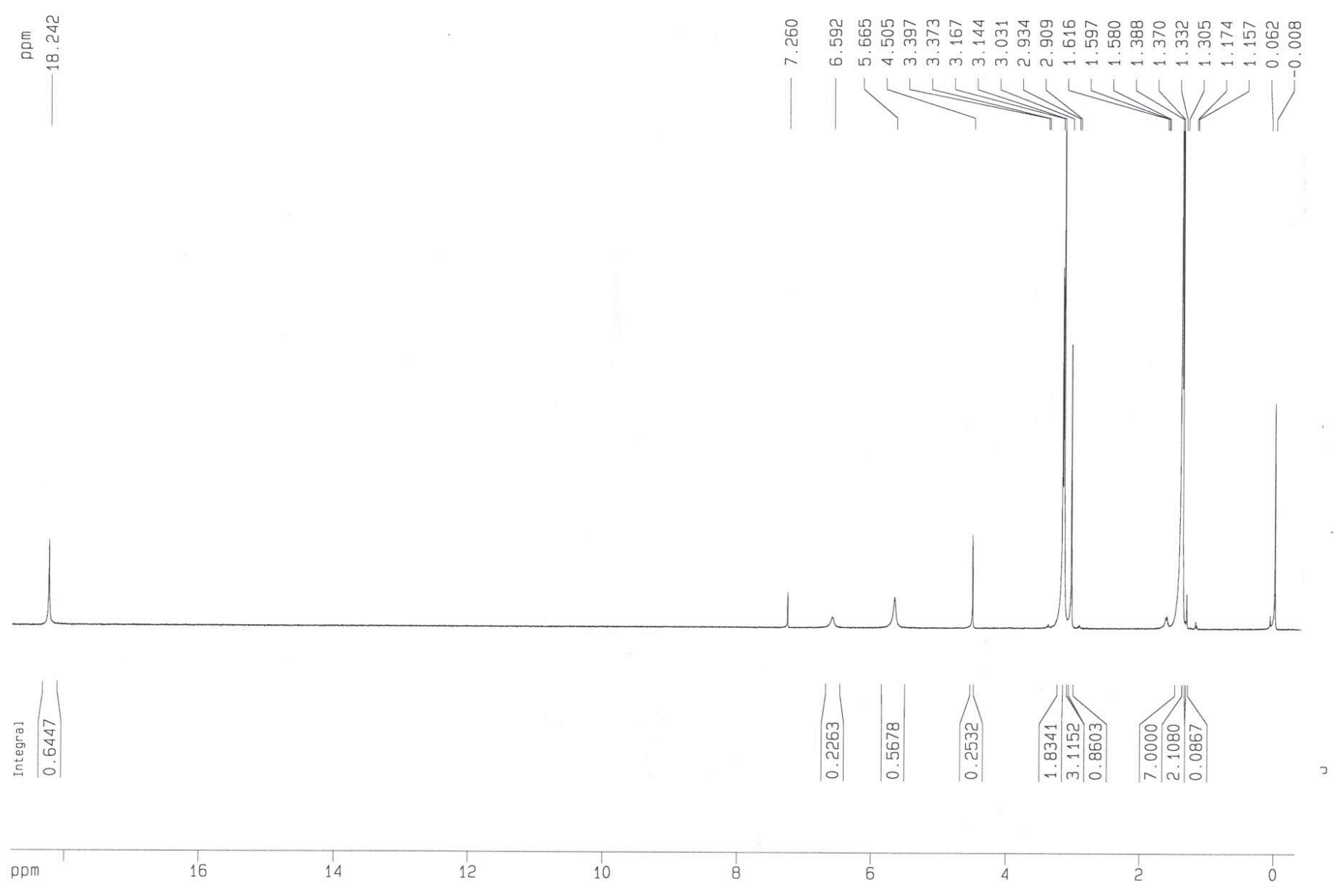


${ }^{1} \mathrm{H}$ NMR of a mixture of $\mathrm{Ph}_{3} \mathrm{CNHCOCH}(\mathrm{CN}) \mathrm{CONMe}_{2}$ and its enol in $\mathrm{CDCl}_{3}$

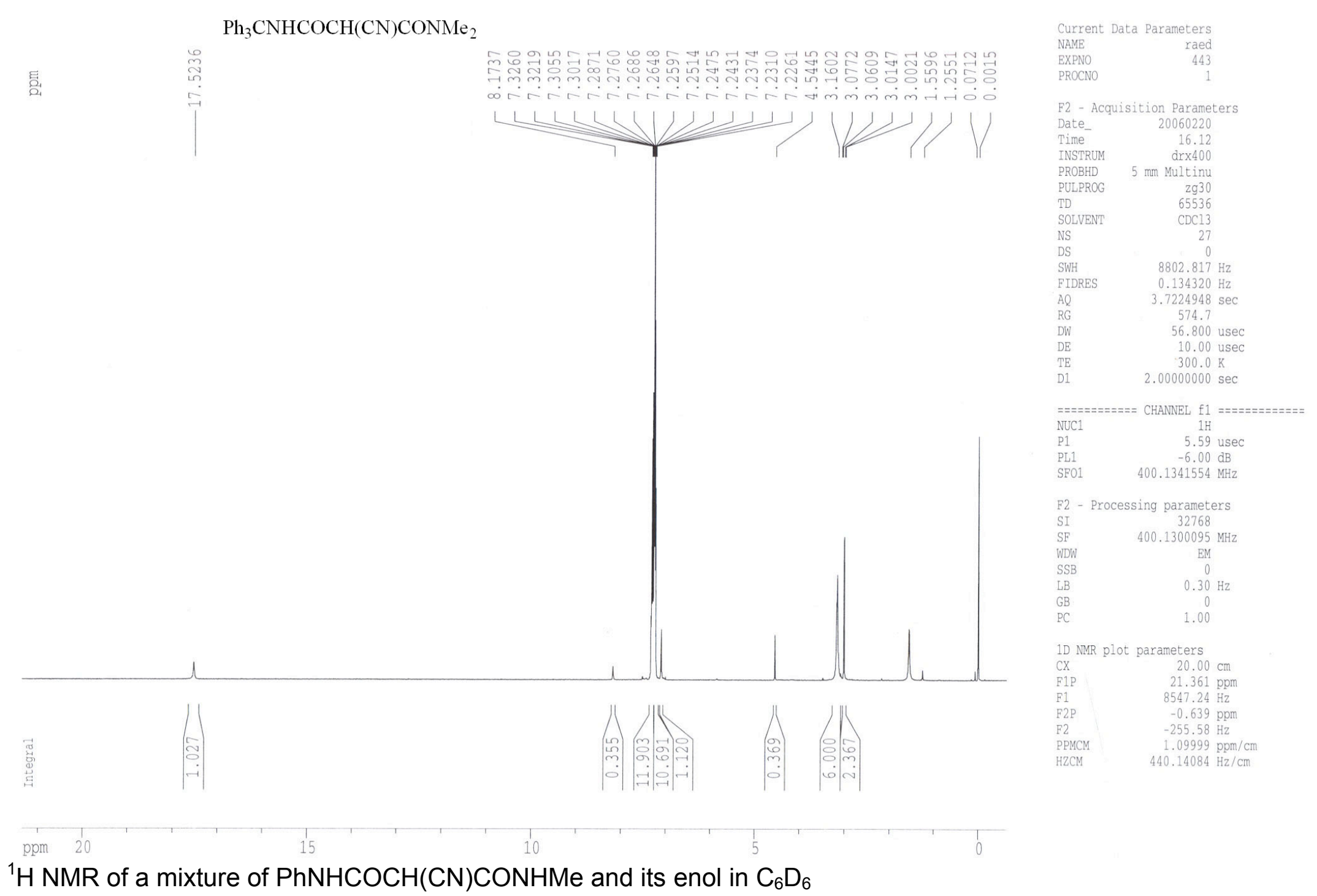




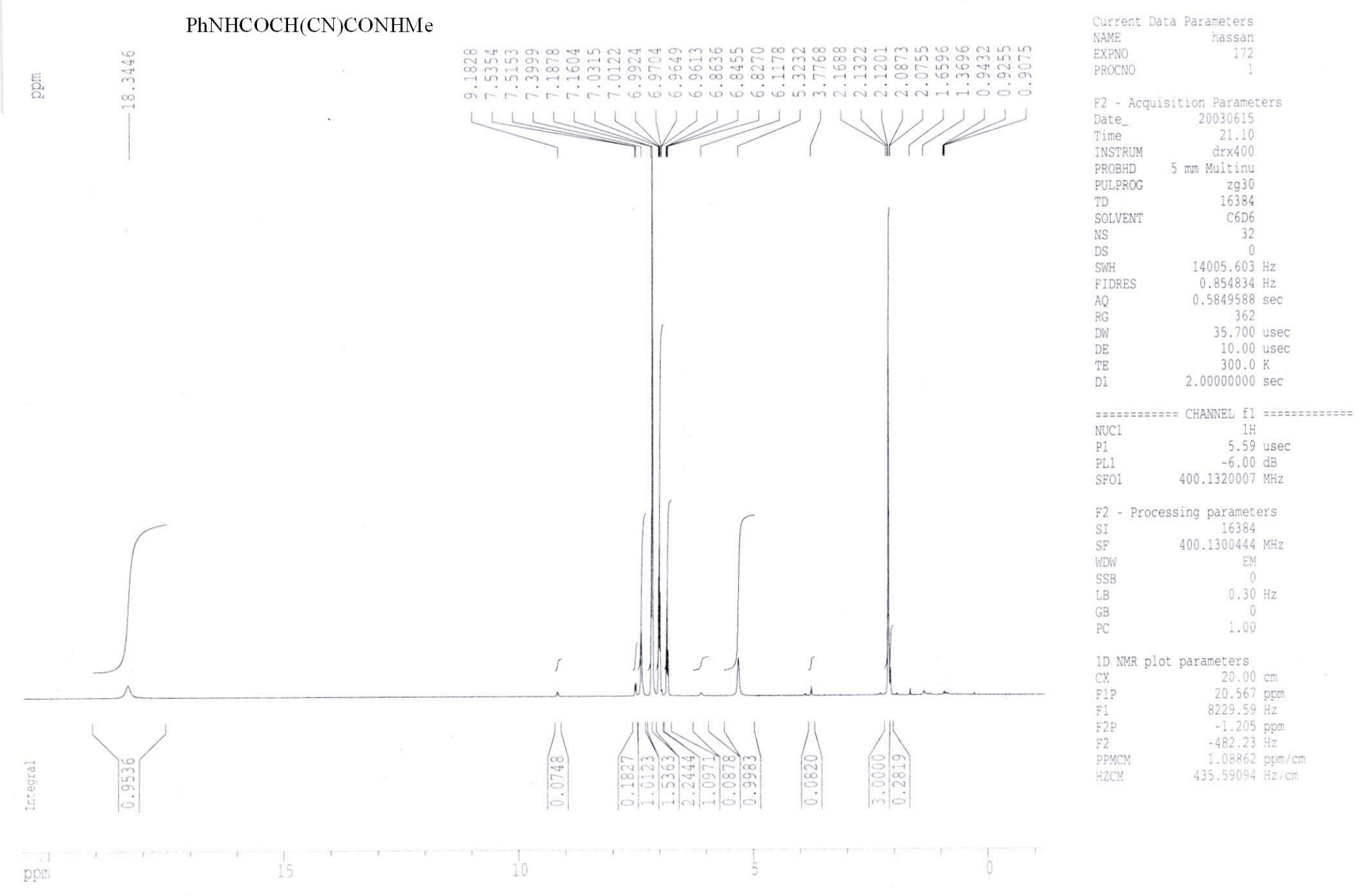

${ }^{1} \mathrm{H}$ NMR of a mixture of $p-\mathrm{AnNHCOCH}(\mathrm{CN}) \mathrm{CONHMe}$ and its enol in $\mathrm{CDCl}_{3}$ 
$p$ - $\mathrm{MeOC}_{6} \mathrm{H}_{4} \mathrm{NHCOCH}(\mathrm{CN}) \mathrm{CONHMe}$

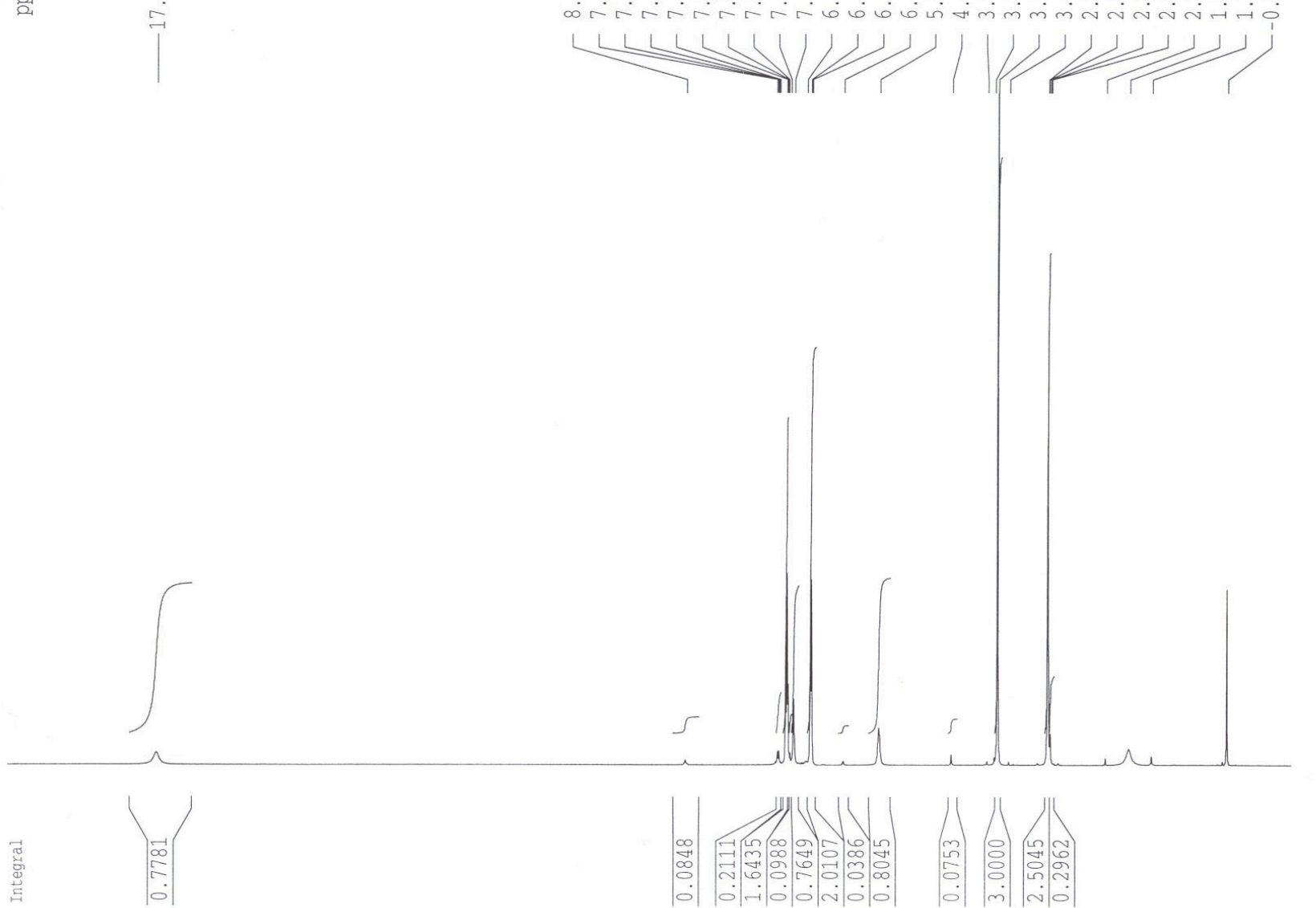

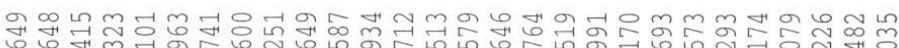

$\therefore$ : i.
Current Data Parameters

EXPNO

F2 - Acquisition Parameters

Date_ $\quad 20030630$

Time $\quad 14.39$

PROBHD $5 \mathrm{~mm}$ Multinu

$\begin{array}{lr}2 \mathrm{~g} 30 \\ \text { TD } & 65536 \\ \text { TD } & \text { CDC13 }\end{array}$

SOLVENT $\quad$ CDC13

SWH $\quad 14005.603 \mathrm{~Hz}$

$0.213709 \mathrm{~Hz}$
$2.3396852 \mathrm{gz}$

$\begin{array}{lc}\text { AQ } & 2.3396852 \mathrm{sec} \\ \text { RG } & 574.7\end{array}$

$\begin{array}{lr}\text { DW } & 35.700 \text { usec } \\ \text { DE } & 10.00 \mathrm{usec}\end{array}$

$300.0 \mathrm{~K}$

$2.00000000 \mathrm{sec}$

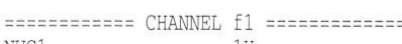

$\begin{array}{lc}\text { NuC1 } & 1 \mathrm{H} \\ \text { P1 } & 5.59 \text { usec }\end{array}$

PL1

- Processing parameters

32768
SF

WDW $\quad$ EM

$\begin{array}{ll}\text { LB } & 0.30 \mathrm{~Hz} \\ \text { GB } & 0\end{array}$

1D NMR plot parameters

$\begin{array}{lr}\text { CX } & 20.00 \mathrm{~cm} \\ \text { F1P } & 20.166 \mathrm{ppm}\end{array}$

$\begin{array}{ll}\text { F1 } & 8068.83 \mathrm{~Hz} \\ \text { F2P } & -1.073 \mathrm{ppm}\end{array}$

$\begin{array}{ll}\text { F2 } & -429.20 \mathrm{~Hz} \\ \text { PPMCM } & 1.06191 \\ \end{array}$

HZCM $\quad 424.06115 \mathrm{ppm} / \mathrm{cm}$ 
${ }^{1} \mathrm{H}$ NMR of a mixture of $\mathrm{C}_{6} \mathrm{~F}_{5} \mathrm{NHCOCH}(\mathrm{CN}) \mathrm{CONHMe}$ and its enol in $\mathrm{CD}_{3} \mathrm{CN}$

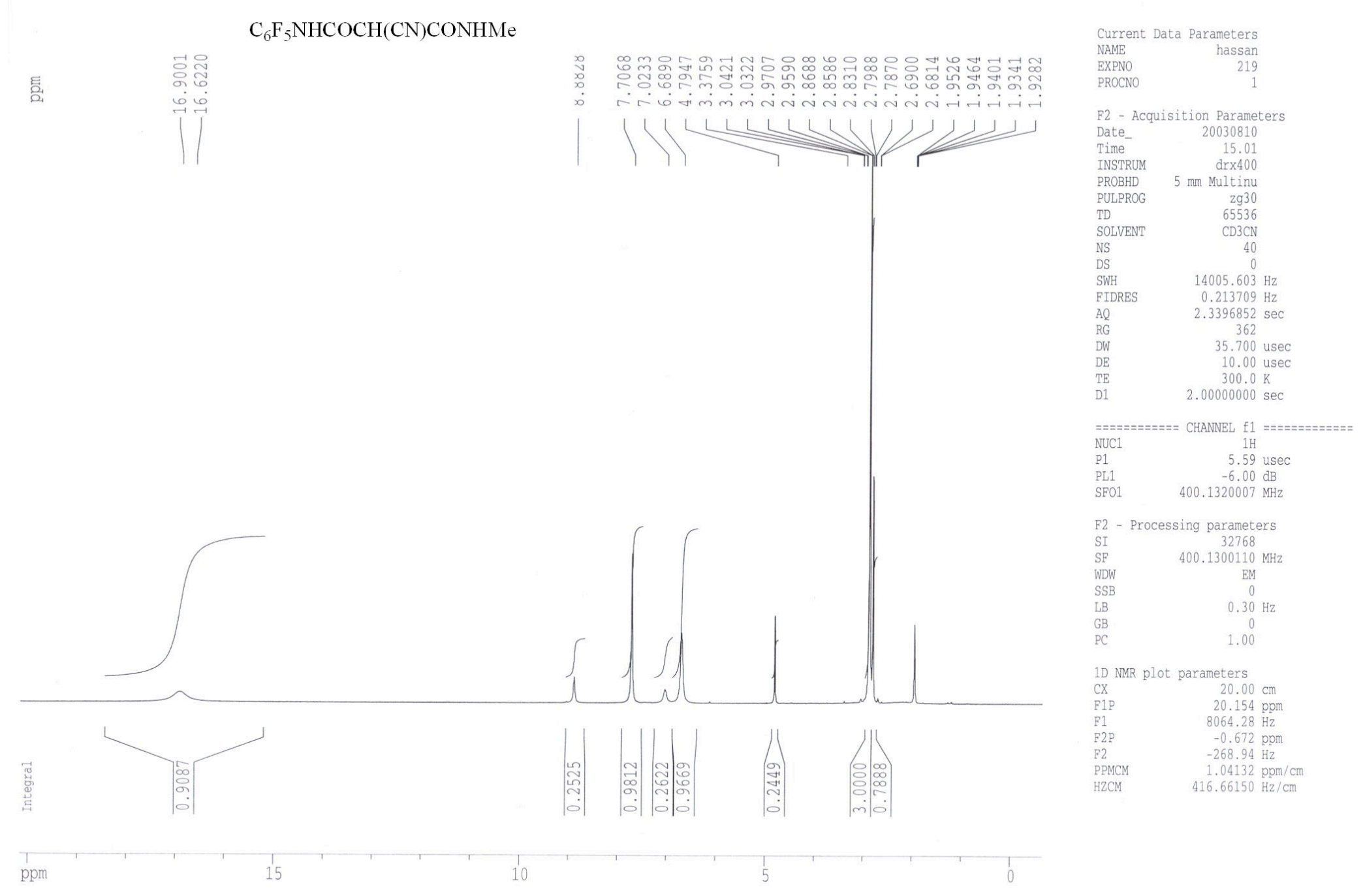


${ }^{1} \mathrm{H}$ NMR of a mixture of $i-\mathrm{PrNHCOCH}(\mathrm{CN}) \mathrm{CONHMe}$ and its enol in $\mathrm{CDCl}_{3}$

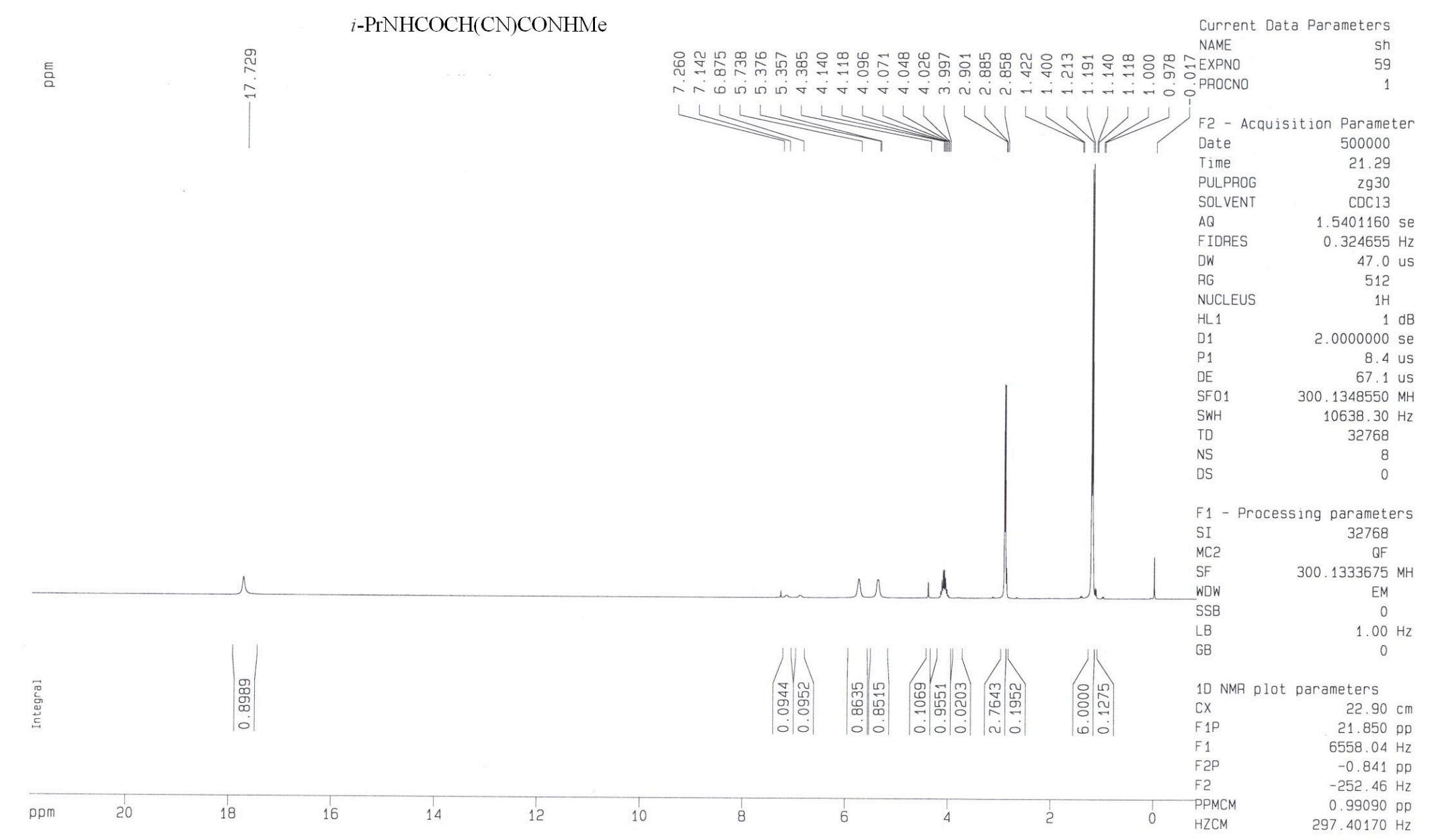


${ }^{1} \mathrm{H}$ NMR of a mixture of $t$-BuNHCOCH$(\mathrm{CN}) \mathrm{CONHMe}$ and its enol in $\mathrm{CDCl}_{3}$

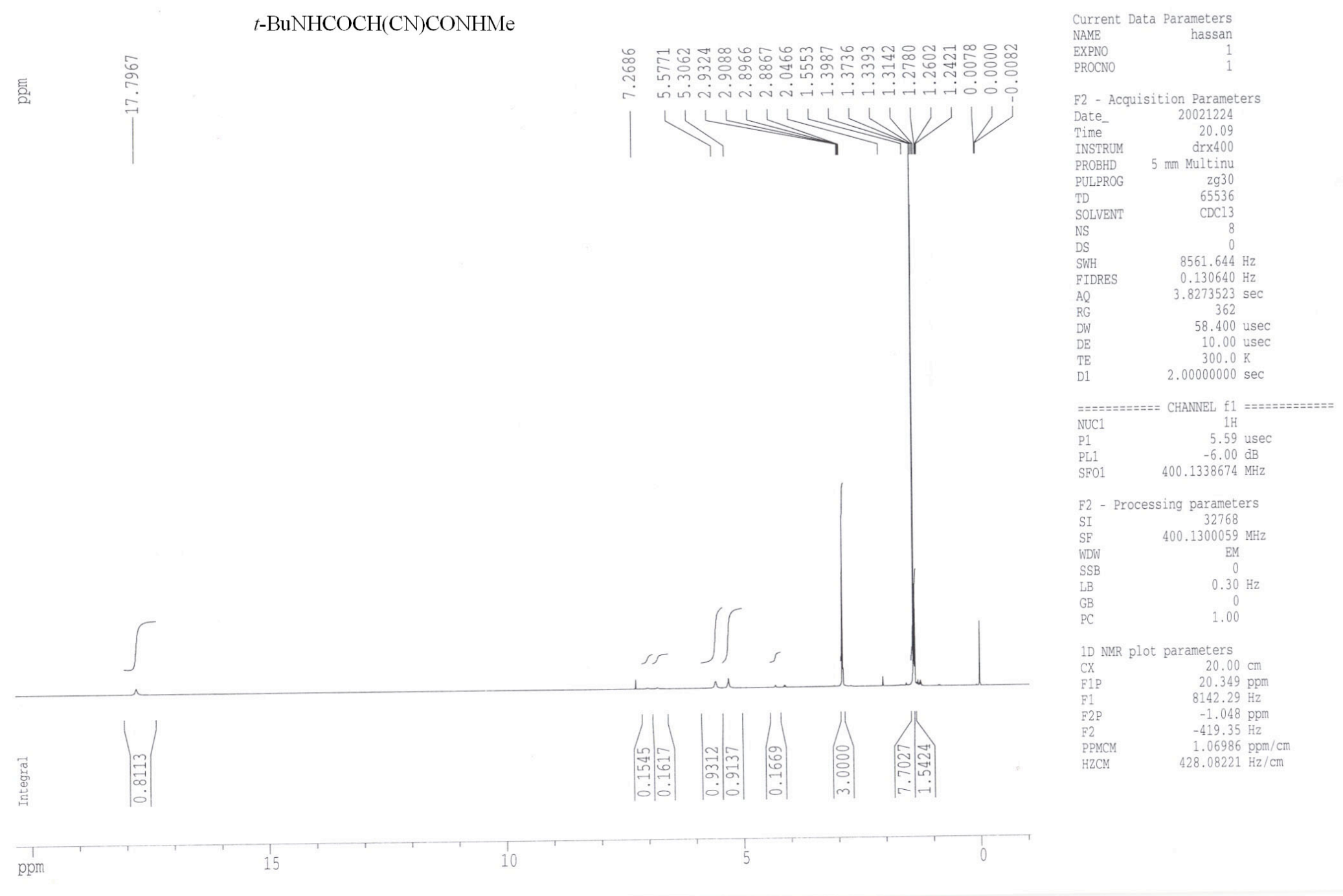


${ }^{1} \mathrm{H}$ NMR of a mixture of $\mathrm{PhNHCOCH}(\mathrm{CN}) \mathrm{CONH}_{2}$ and its enol in $\mathrm{CD}_{3} \mathrm{CN}$

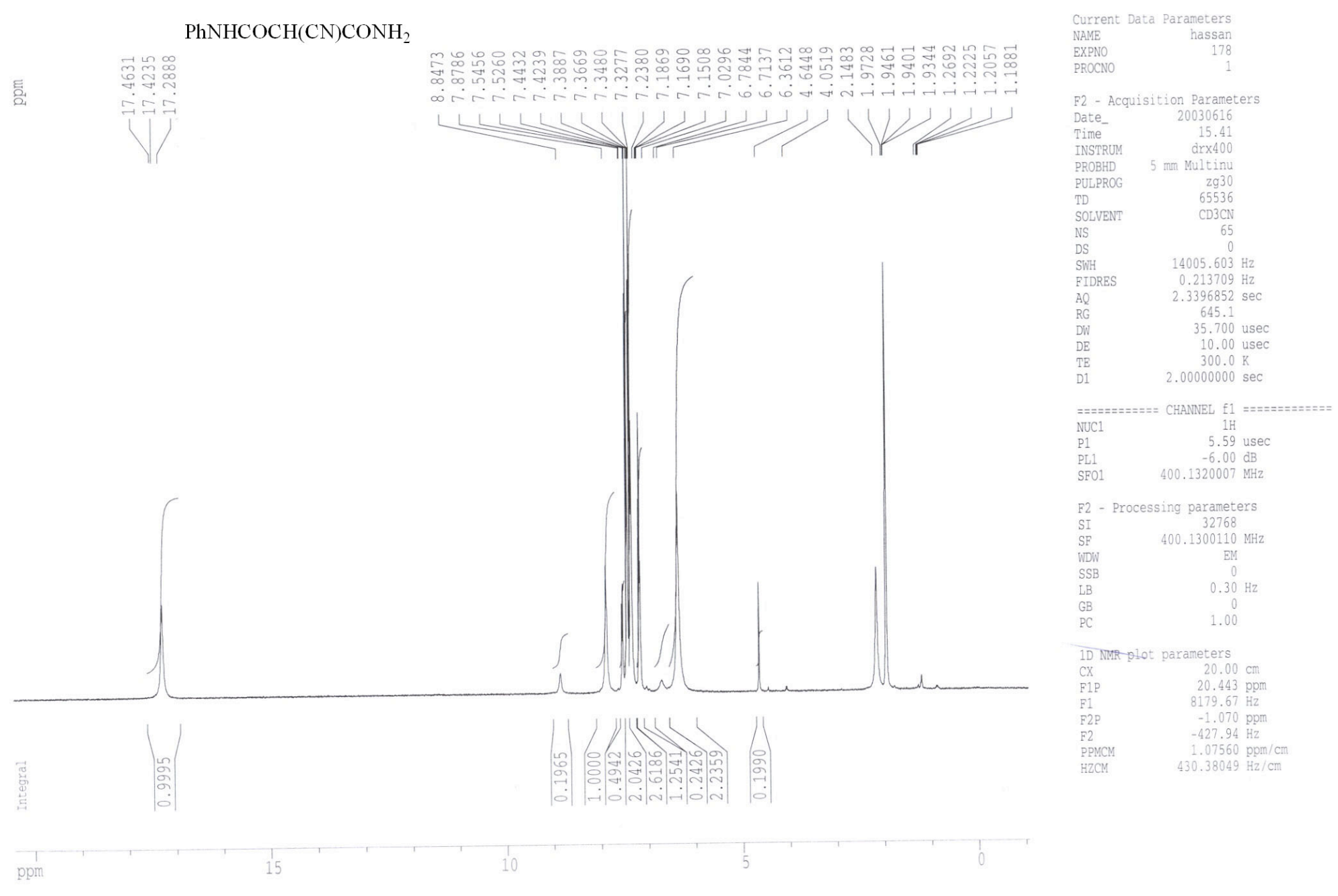

${ }^{1} \mathrm{H}$ NMR of a mixture of $p-\mathrm{AnNHCOCH}(\mathrm{CN}) \mathrm{CONH}_{2}$ and its enol in THF-d $\mathrm{d}_{8}$ 


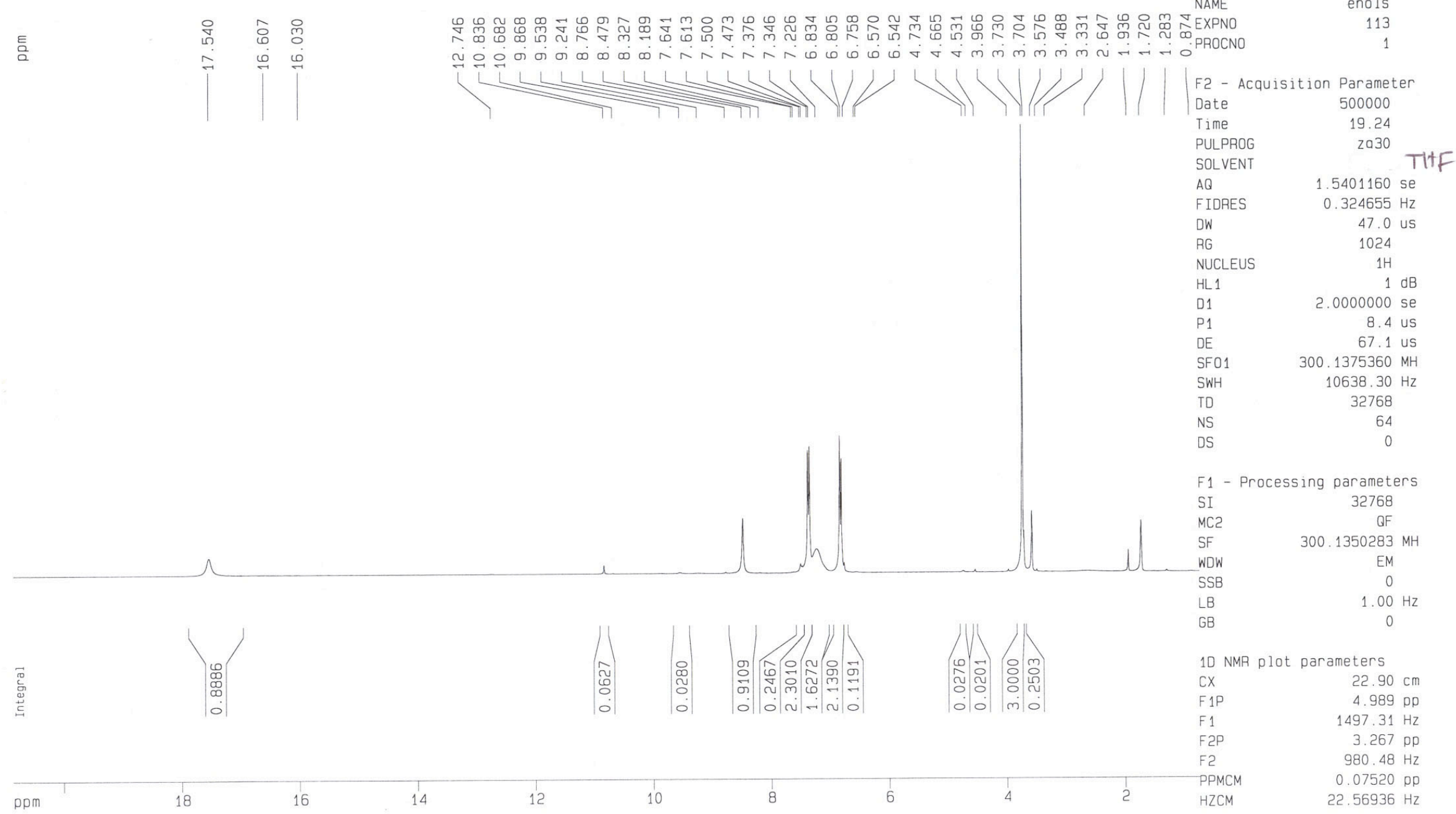

${ }^{1} \mathrm{H}$ NMR of a mixture of $\mathrm{C}_{6} \mathrm{~F}_{5} \mathrm{NHCOCH}(\mathrm{CN}) \mathrm{CONH}_{2}$ and its enol in THF-d 
$\mathrm{C}_{6} \mathrm{~F}_{5} \mathrm{NHCOCH}(\mathrm{CN}) \mathrm{CONH}_{2}$

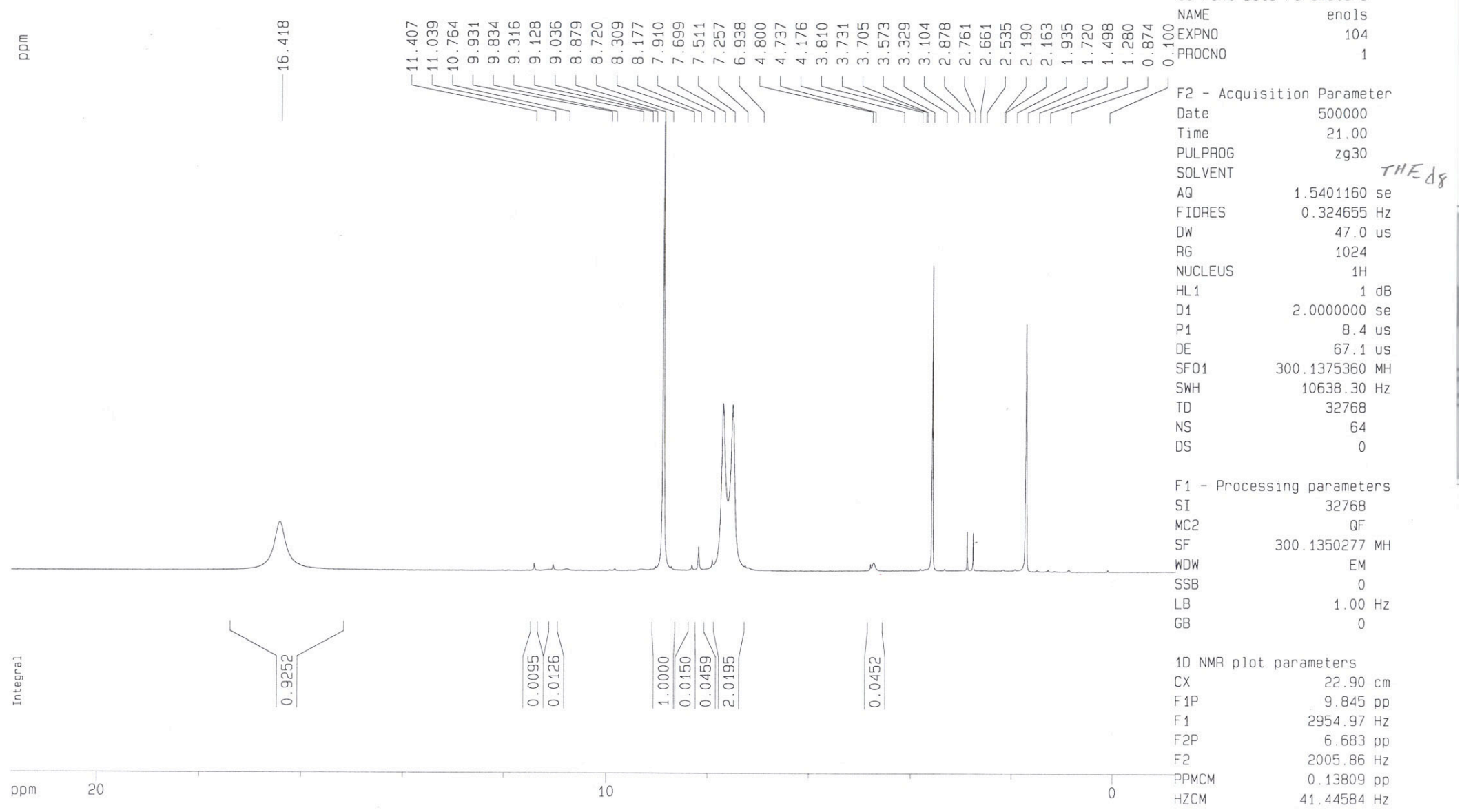

${ }^{1} \mathrm{H}$ NMR of a mixture of $i-\mathrm{PrNHCOCH}(\mathrm{CN}) \mathrm{CONH}_{2}$ and its enol in $\mathrm{CDCl}_{3}$ 
$i-\mathrm{PrNHC}(\mathrm{OH})=\mathrm{C}(\mathrm{CN}) \mathrm{CONH}_{2}$

言

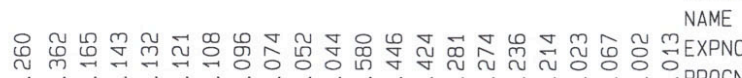

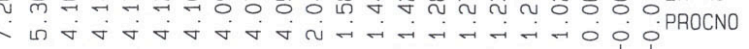

$\longrightarrow$ W

$\begin{array}{rr}\text { TIme } & 13.27 \\ \text { PULPROG } & 2930\end{array}$

SOLVENT CDC13

AQ $\quad 1.5401160 \mathrm{se}$

DW 47.0 Us

RG 4096

HL1 1 1H

$\begin{array}{lll}H L 1 & 1 \\ D 1 & 2.0000000 & d B\end{array}$

P1 8.4 us

$\begin{array}{lr}\text { DE } & 67.1 \text { US } \\ \text { SF01 } & 300.1348550 \mathrm{MH}\end{array}$

SWH

$\begin{array}{ll}\text { SWH } & 10638.30 \\ \text { TO } & \end{array}$

NS $\quad 320$

F1 - Processing parameters

SI 32768

$\begin{array}{ll}\text { MC2 } & \text { QF } \\ \text { MF } & 300.1333675\end{array}$

$\begin{array}{ll}\text { WDW } & \text { no } \\ \text { SSB } & 0.00 \text { He }\end{array}$
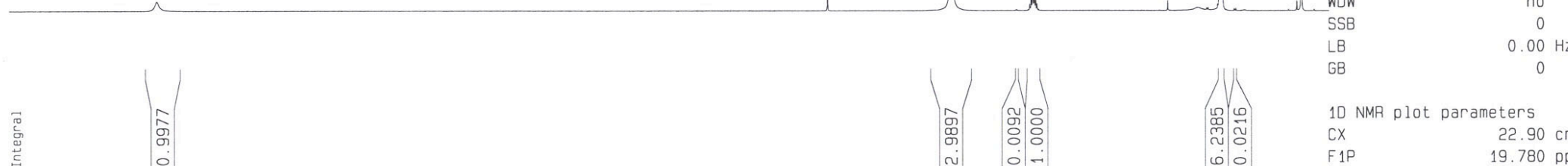

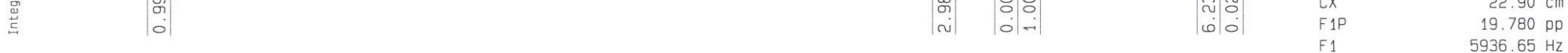

$5936.65 \mathrm{~Hz}$
$F-0.427$

F2 $-128.18 \mathrm{~Hz}$

ppm

16

14

12

10

8

6

4

2

0 HZCM

264.83948 H 
${ }^{1} \mathrm{H}$ NMR of a mixture of $t$-BuNHCOCH(CN)CONH${ }_{2}$ and its enol in $\mathrm{C}_{6} \mathrm{D}_{6}$

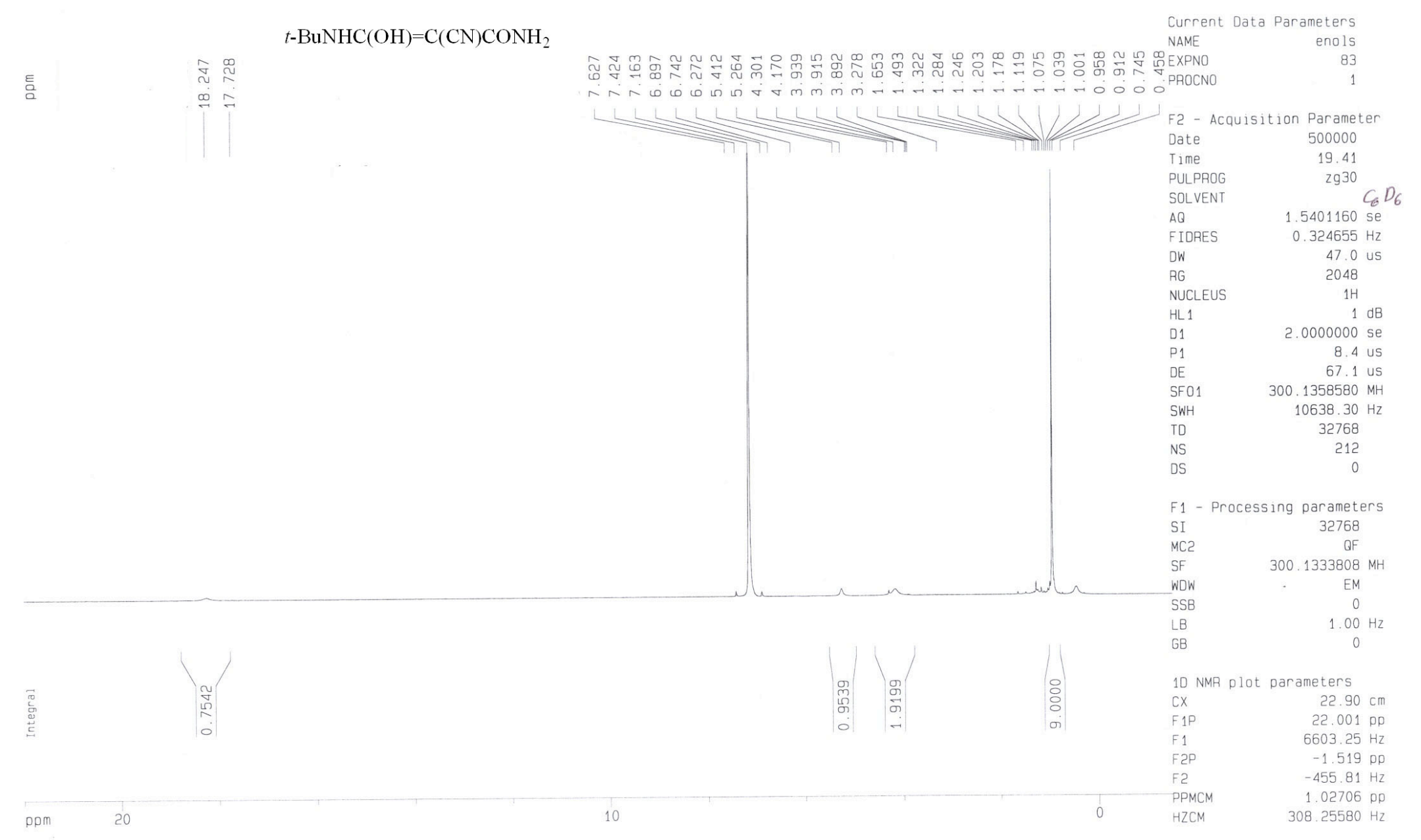


${ }^{1} \mathrm{H}$ NMR of a mixture of $\mathrm{C}_{6} \mathrm{~F}_{5} \mathrm{NHCOCH}(\mathrm{CN}) \mathrm{CONHPh}$ and its enol in THF-d

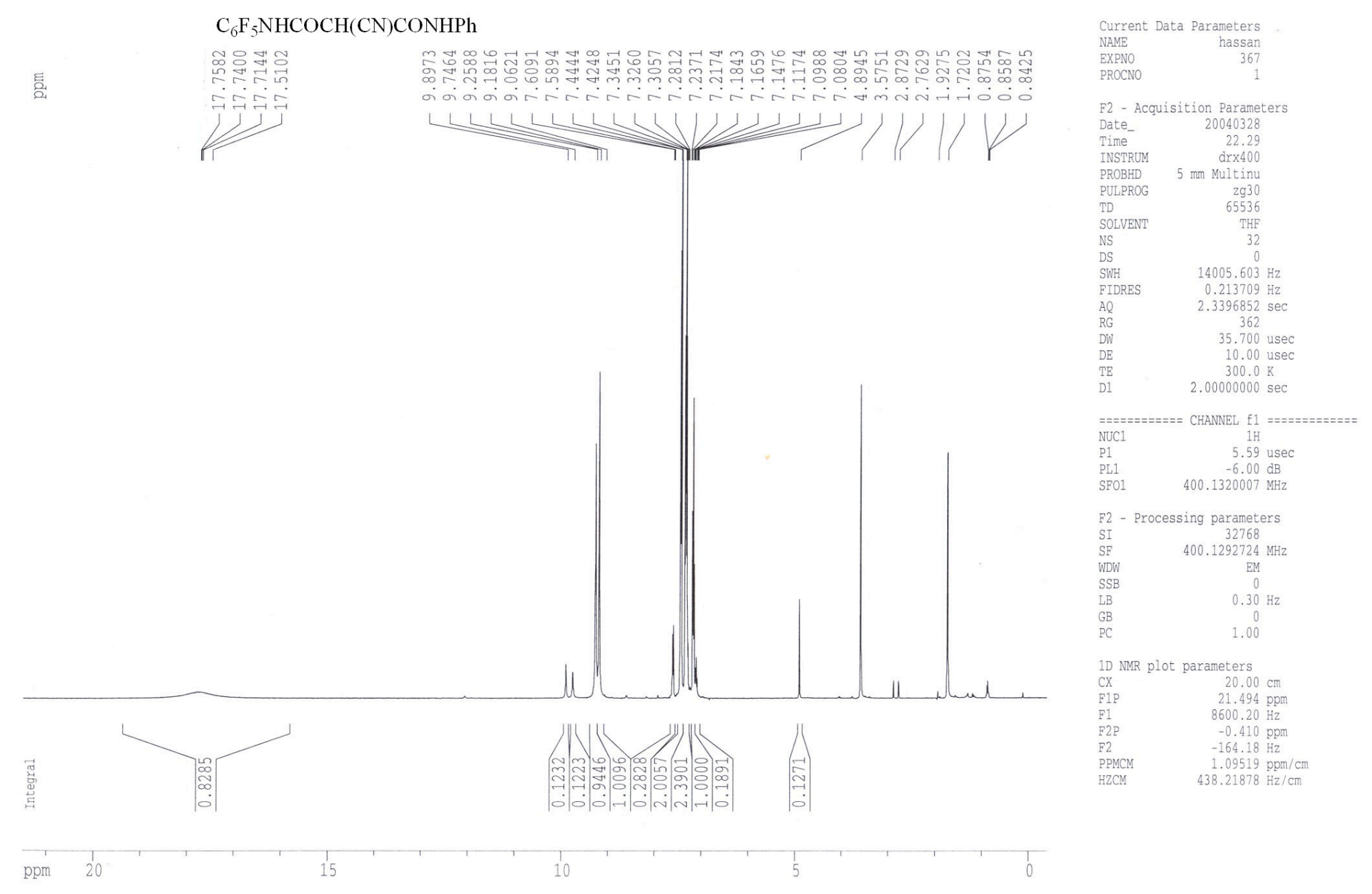


${ }^{1} \mathrm{H}$ NMR of a mixture of $\mathrm{PhNHCOCH}(\mathrm{CN}) \mathrm{CONHPh}$ and its enol in THF-d

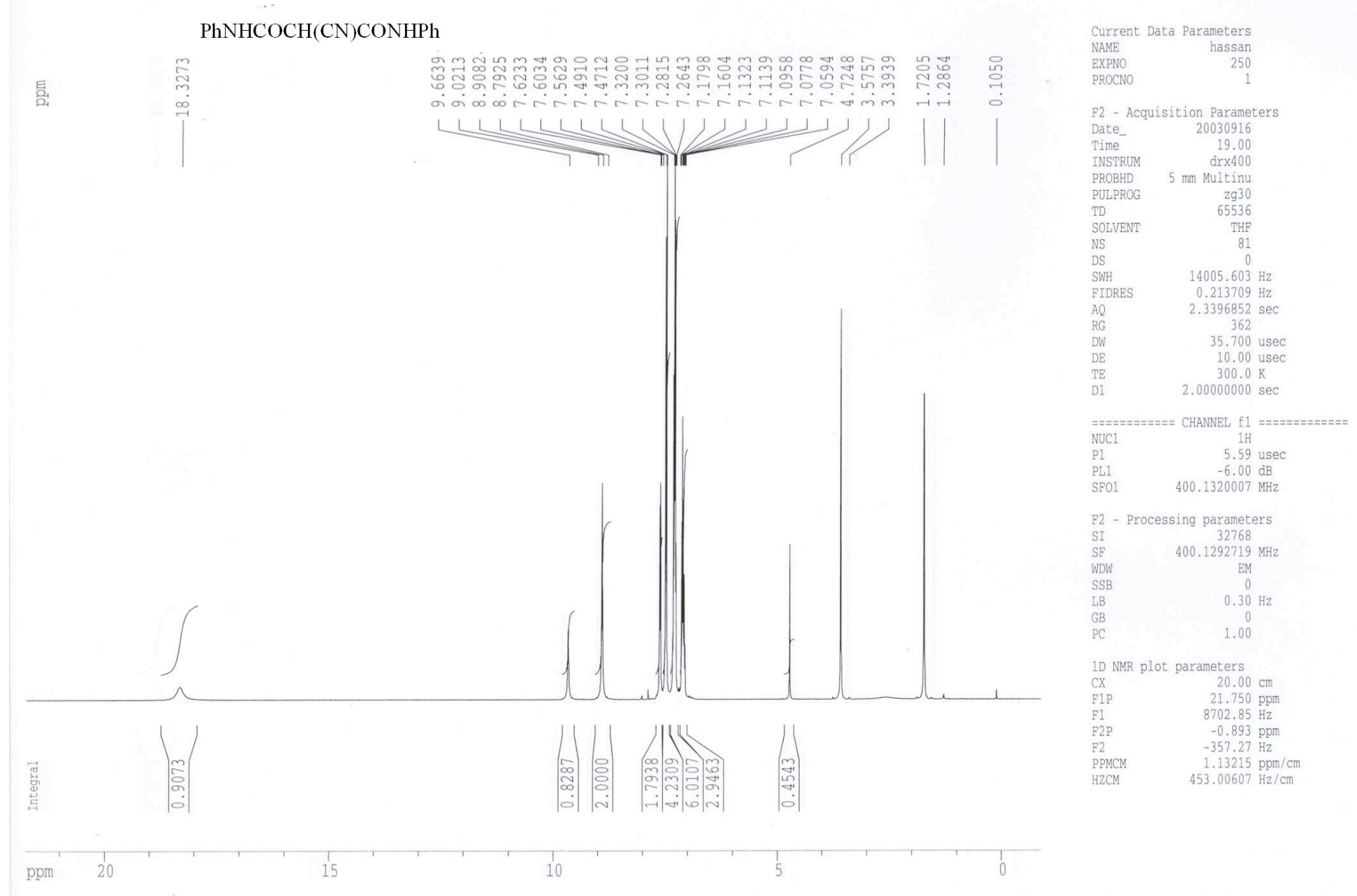


${ }^{1} \mathrm{H}$ NMR of a mixture of $i-\mathrm{PrNHCOCH}(\mathrm{CN}) \mathrm{CONHMe}$ and its enol in THF- $\mathrm{d}_{8}$

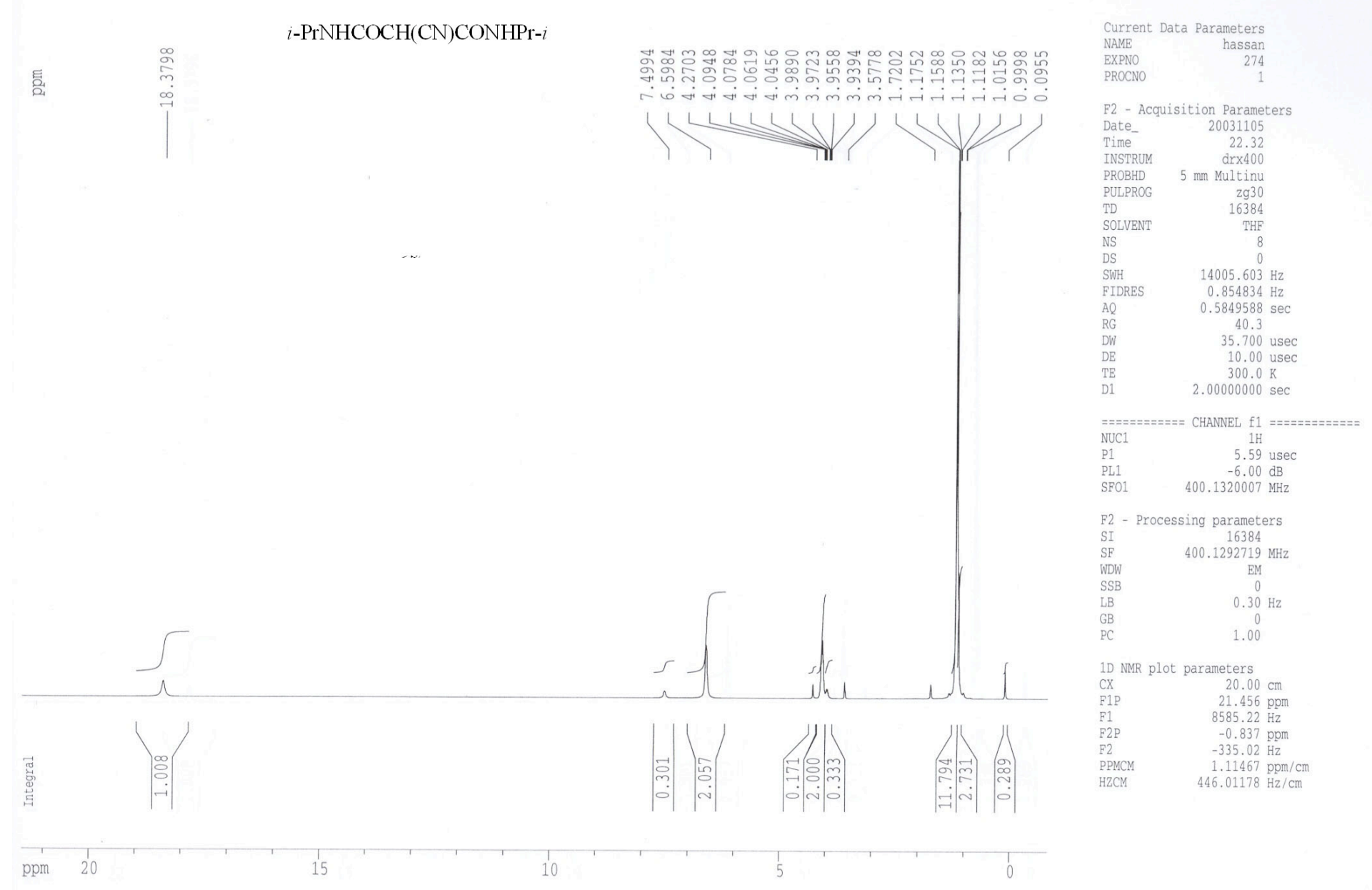


${ }^{1} \mathrm{H}$ NMR of a mixture of $\mathrm{Ph}_{3} \mathrm{CNHCOCH}(\mathrm{CN}) \mathrm{CONMe}_{2}$ and its enol in DMF-d

$\mathrm{Ph}_{2} \mathrm{CHNHCOCH}(\mathrm{CN}) \mathrm{CONHCHPh}_{2}$
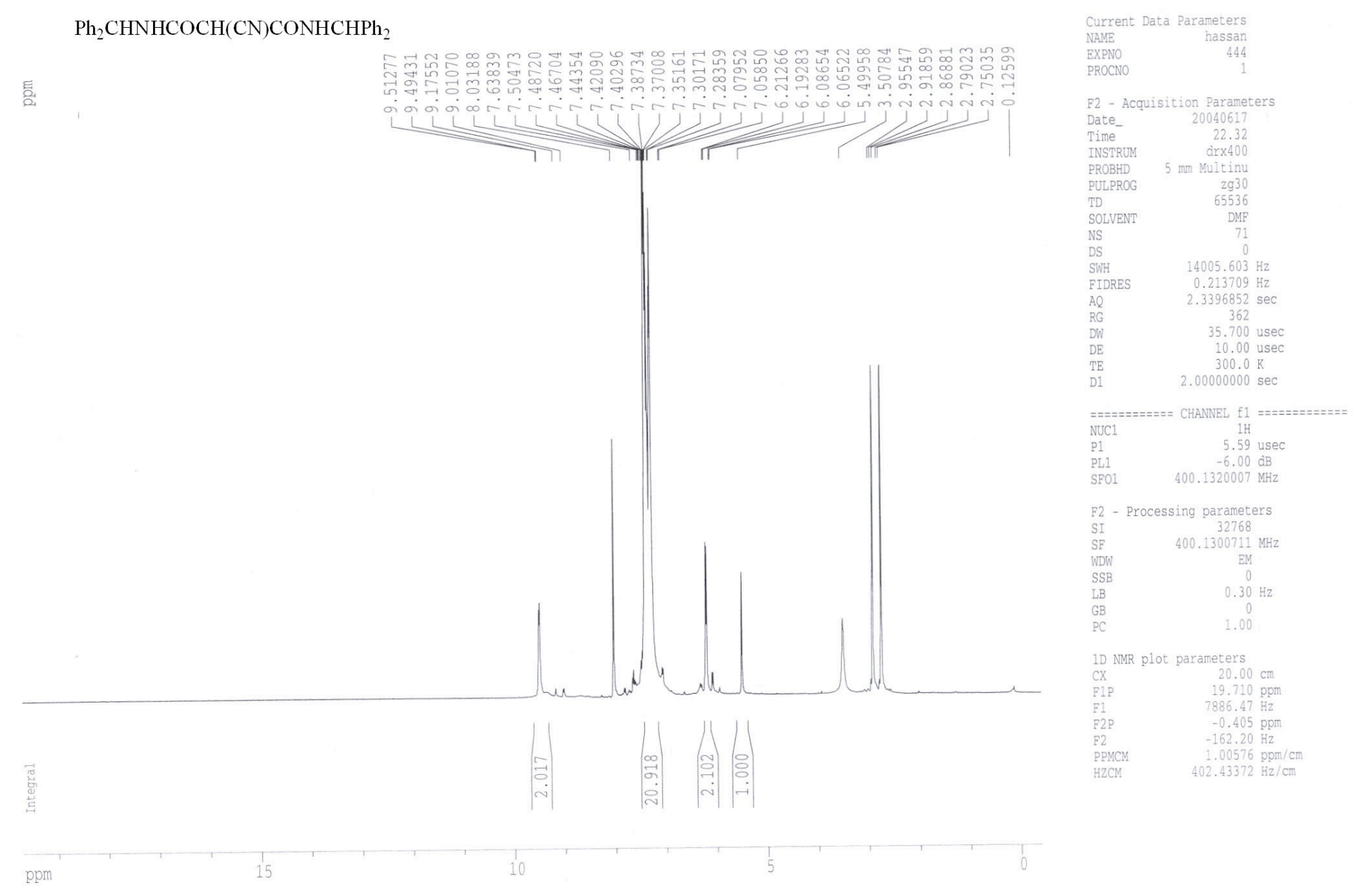